\title{
LOOK AT HER: ANALYSING THE DEPICTION OF WOMEN ON THE COVERS OF WOMEN'S MAGAZINES FROM 1975-2015
}

By

Sophie Beaumont

A thesis

submitted to Victoria University of Wellington in fulfilment of the requirements for the degree of Master of Arts, in Criminology

School of Social and Cultural Studies 


\section{Abstract}

Women's magazines have a role in constructing and defining what it means to be a woman. Deciphering messages in mediums specifically designed for women is therefore key to understanding what women may be learning about femininity. This thesis examines the depiction of women in women's magazines, focussing on sexualisation and the portrayal of traditional gender roles. Traditional gender stereotypes and the sexual objectification of women are key mechanisms contributing to the subordinate position of women in society. This thesis argues that alongside their contributions to gender inequality, such depictions can also reinforce ideas that sustain rape culture with the latter referring to a climate where sexual violence is normalised and trivialised. By conducting a longitudinal content analysis (19752015) of cover pages from New Zealand women's magazines, this thesis investigates whether there are any changes in the level of sexualisation and depiction of traditional gender roles across prominent women's magazines. The findings of this thesis indicate that overall there is a low level of sexualisation present in cover page images from women's magazines, and significant differences exist both between publications as well as across the four decades of analysis. The depiction of traditional gender roles is consistent across the time period studied, and when such gender stereotypes are present they remove agency from women reducing them to 'decorative' objects within images. Messages suggesting women should be sexualised and decorative may reinforce ideas central to gender inequality, rape culture, and sexual violence against women. The implications of this thesis suggest that women's magazines may not be a safe space for readers to celebrate their gender - rather, such publications may influence ideas that facilitate gender inequality. 


\section{Acknowledgements}

I would firstly like to express my appreciation to my two wonderful supervisors Jan Jordan and Sarah Wright. Your guidance and support throughout this process has been invaluable. Your combined wisdom helped me to 'wrestle the beast' and get this thesis completed. I am so grateful to have had the opportunity to work alongside you both and I cannot thank you enough for coming on this journey with me.

To the staff and students of Murphy Levels 9 and 11, thank you for your support throughout this process - I have loved being part of this department and have enjoyed working alongside you all. Specific thanks go to Dr Russil Durrant for your assistance with my quantitative analysis, I am grateful to you for taking the time to help me figure this section out. Also to Jess, my reliability coder, thank you for your help with this project and I wish you all the best for your own thesis.

On a more personal note, I would like to thank my family, your love and support are the reason I am who I am, and have done what I've done. Thank you for your unwavering confidence in my ability to achieve anything I set my mind to.

To my friends, thank you for being amazing human beings. Each and every one of you is an inspiration and you have all done your part to keep me sane through this process. Two for one eggs, 12.30 lunches, and Friday night drinks are probably why I am still functioning like a (mostly) normal person at this point!

Special thanks go to my flatmate Chelsea, not only for making sure I escape from the bubble of my thesis every now and then, but for joining me in that bubble whenever I needed to rant about something or my chapters needed proofreading.

Lastly, I am deeply grateful for the monetary support provided by the Royal Society of New Zealand Marsden Fund, which helped this project become a reality. 


\section{Table of Contents}

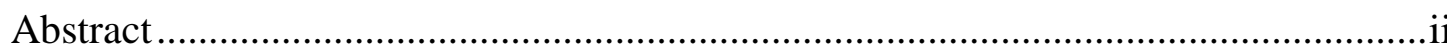

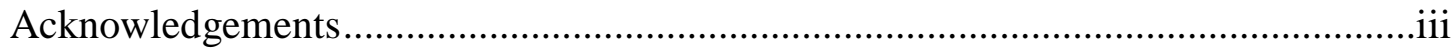

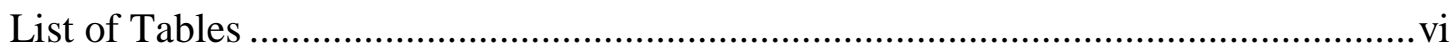

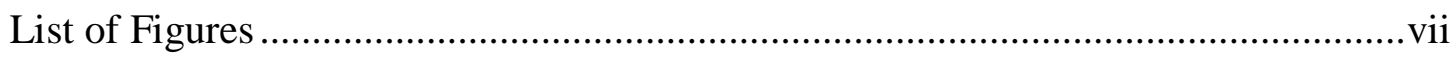

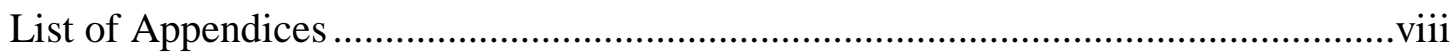

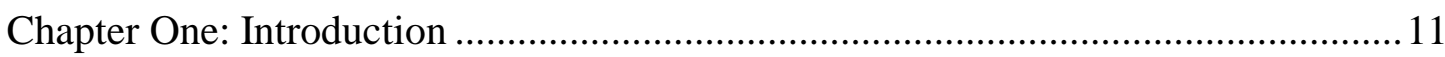

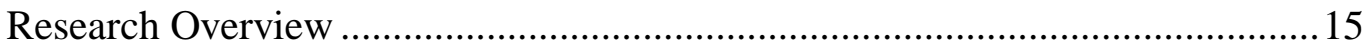

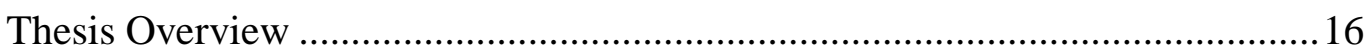

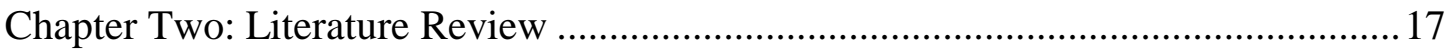

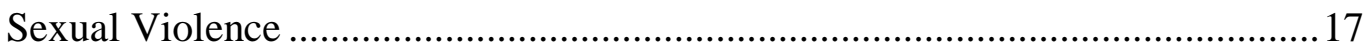

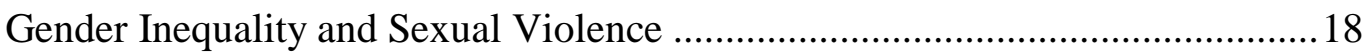

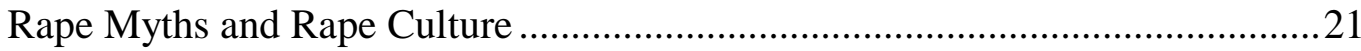

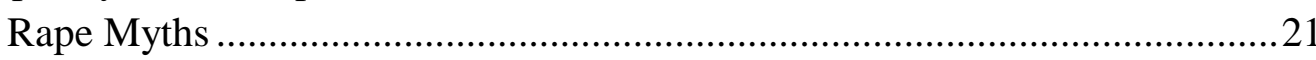

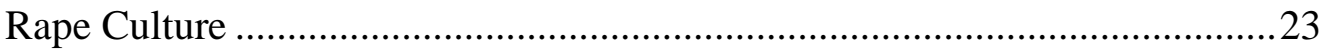

Rape Culture and the Media .....................................................................25

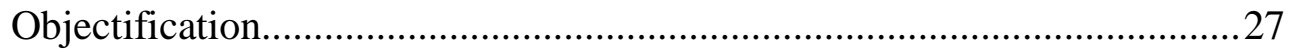

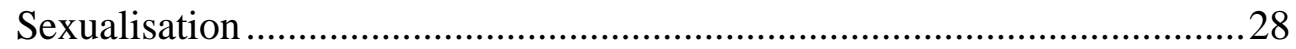

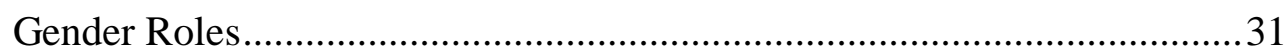

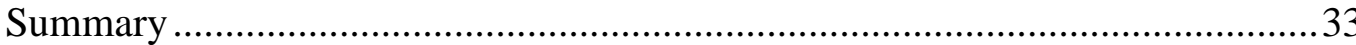

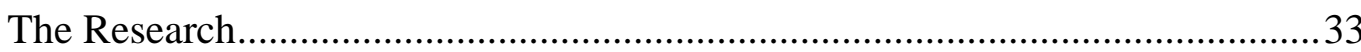

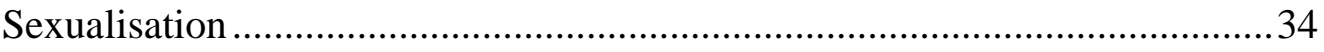

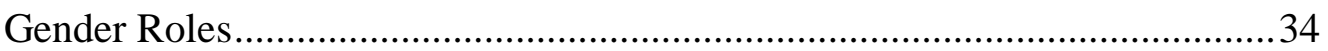

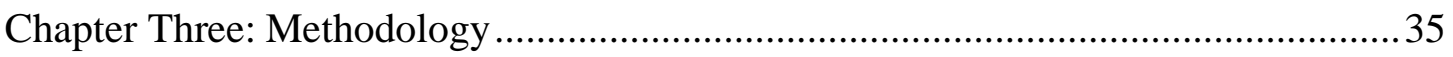

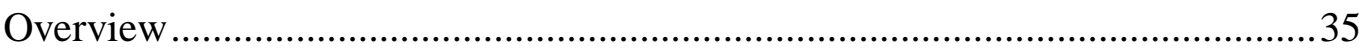

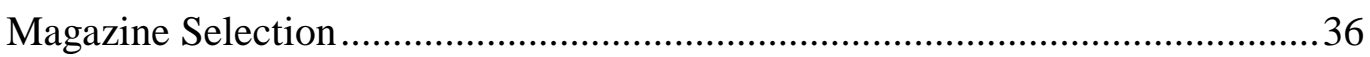

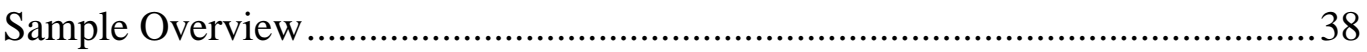

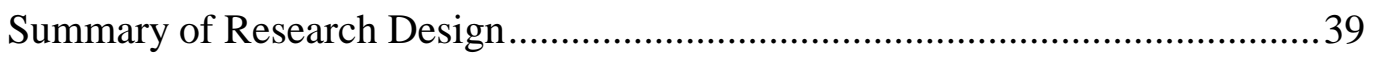

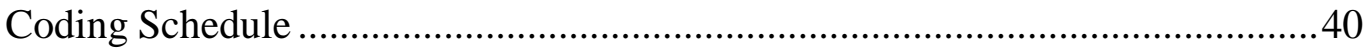

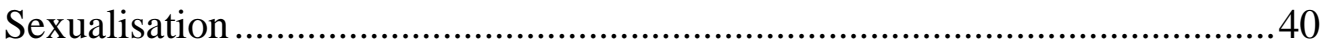

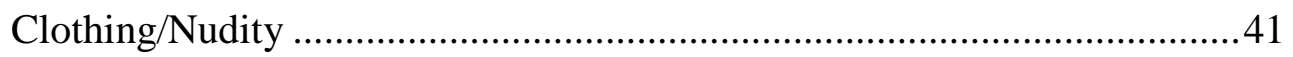

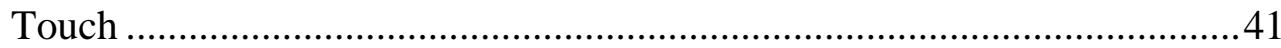

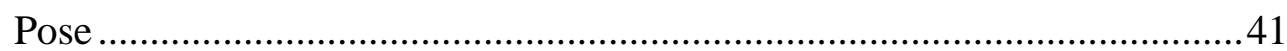

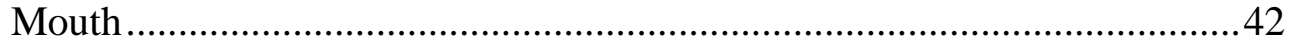

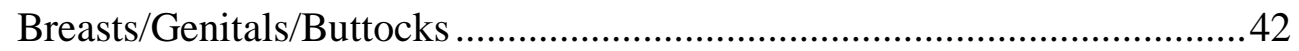

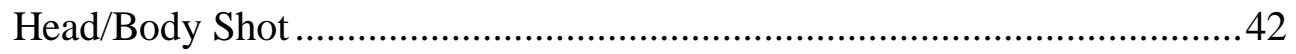

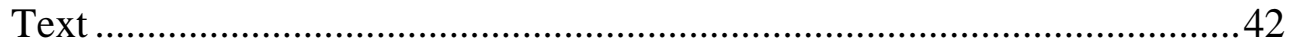

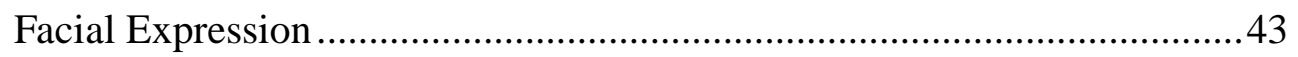

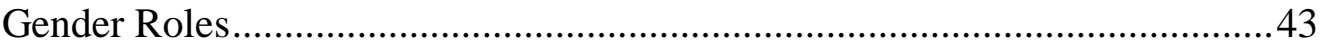

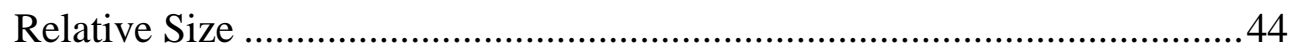

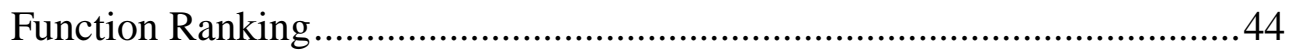

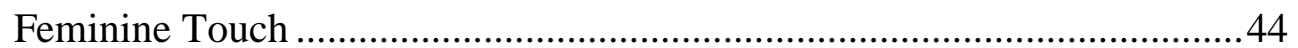

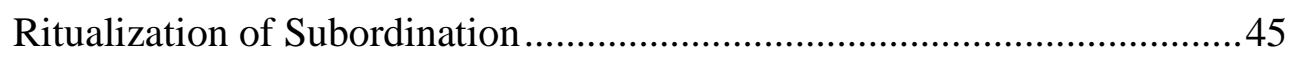

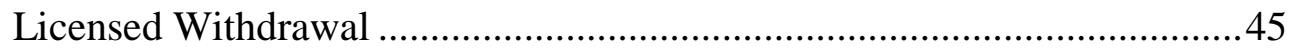




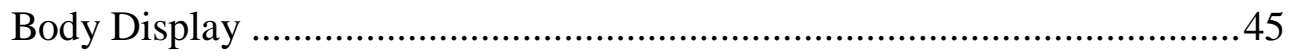

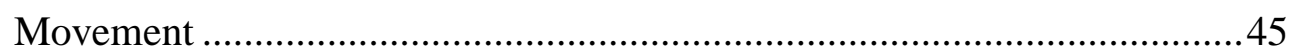

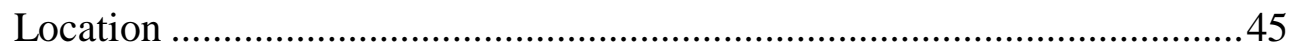

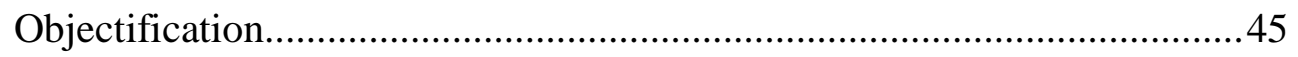

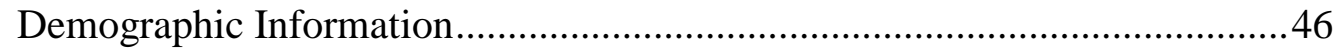

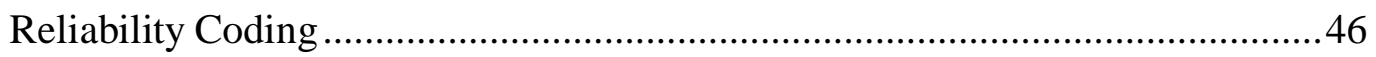

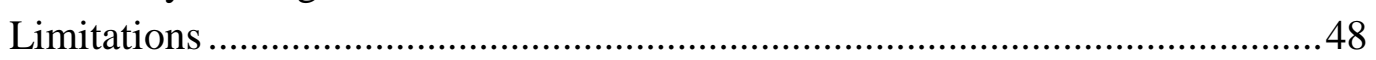

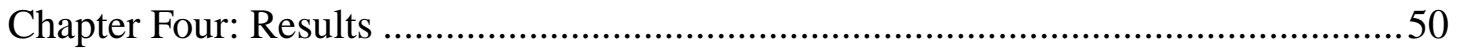

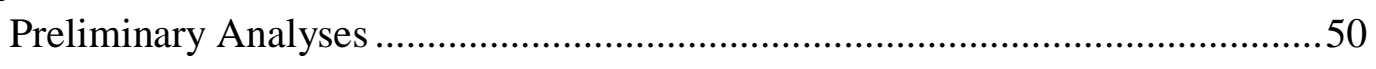

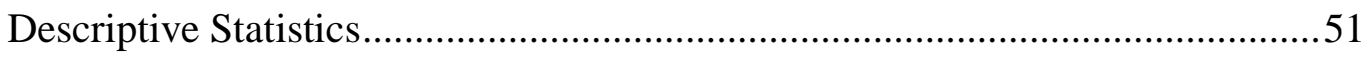

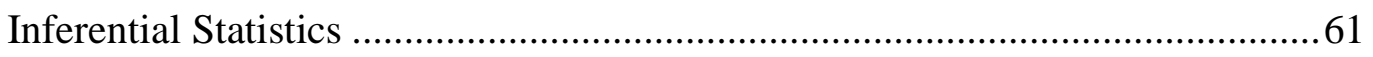

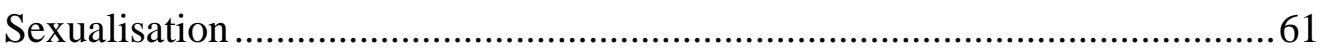

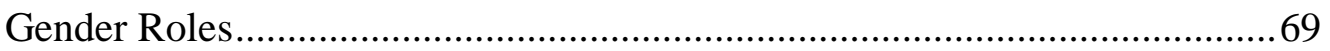

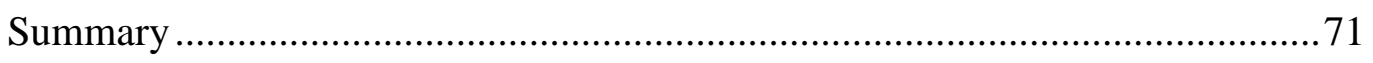

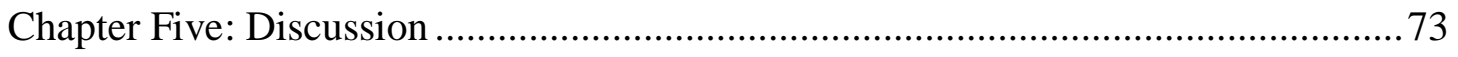

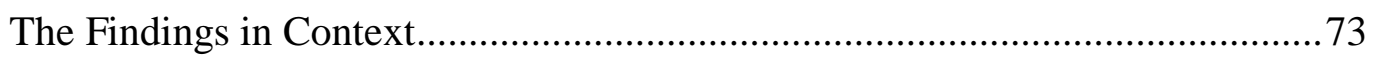

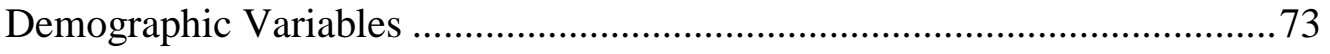

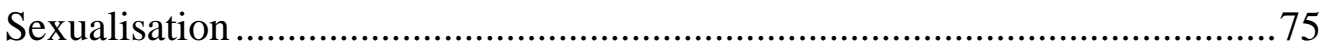

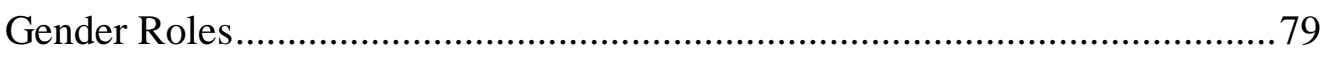

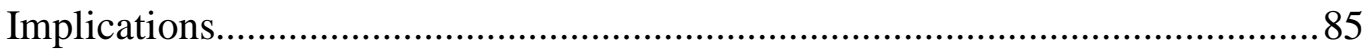

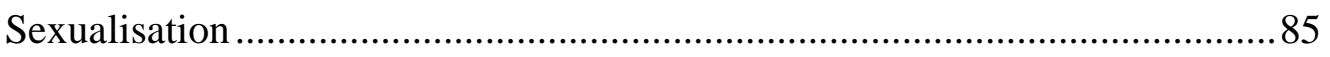

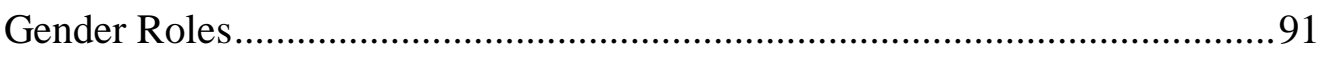

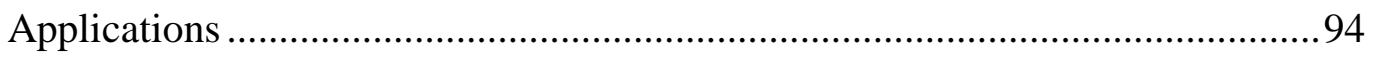

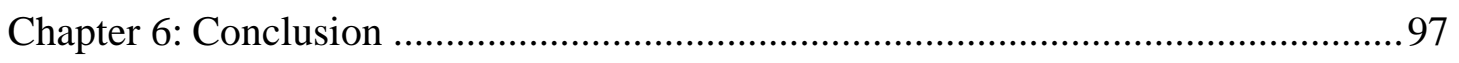

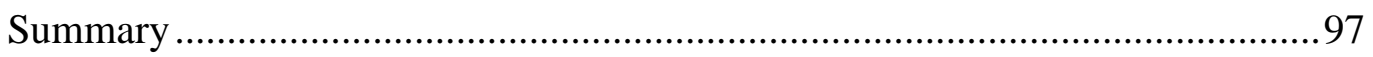

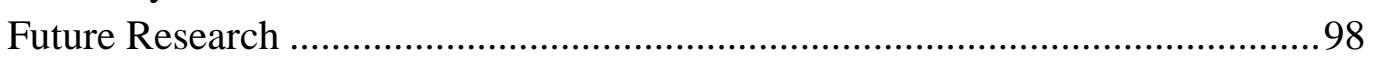

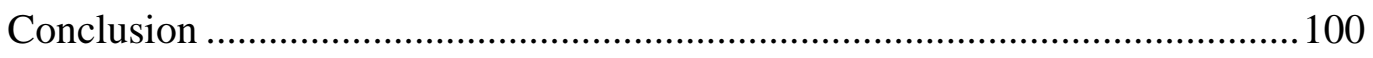

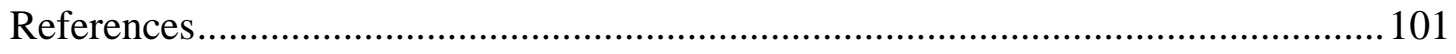

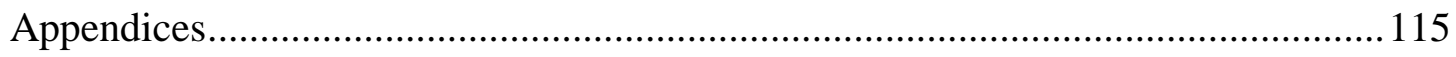

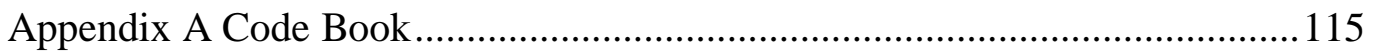

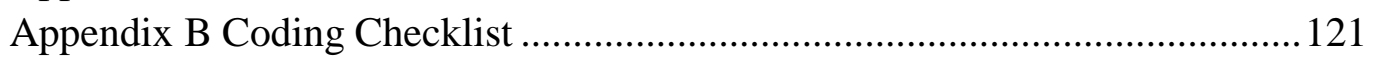




\section{List of Tables}

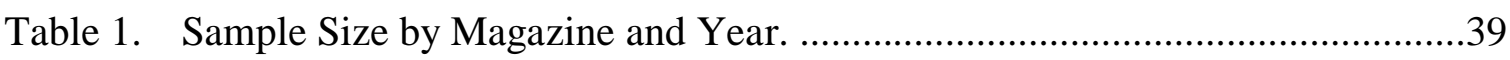

Table 2. Percentage Agreement and Cohen's Kappa Results by Coding Category. ......47

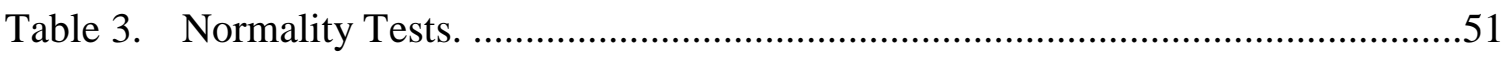

Table 4. Frequency Data for Demographic Variables across Year Groups. ...................52

Table 5. Frequency Data for Each Sexualisation Code across Year Groups. .................55

Table 6. Frequency Data for Each Gender Role Code across Year Groups. ...................58

Table 7. Descriptive Statistics for Sexualisation and Gender Role Scores across

Magazines and Year Groups. ....................................................................61 


\section{List of Figures}

Figure 1. Example of the two most common facial expression codes. ...........................53

Figure 2. Example cover pages for mean scores across year groups. .............................63

Figure 3. Example cover pages for mean scores across publications. ............................64

Figure 4. Example Cosmopolitan cover pages with average sexualisation scores from 1985, 2005, and 2015.

Figure 5. Example CLEO cover pages with average sexualisation scores from 1975, $1985,1995,2005$, and 2015.

Figure 6. Example Cosmopolitan cover pages with average gender role scores from 1985, 1995, 2005, and 2015.

Figure 7. Comparison of average sexualisation scores from Cosmopolitan and NZWW.

Figure 8. Example cover pages where feminine touch is present and not present. .82

Figure 9. Example cover pages where location was coded for and where it was not coded for.

Figure 10. Example cover pages where objectification was coded and where it was not coded.

Figure 11. Comparison of images with agency removing codes present and not present. 92

Figure 12. Cover image from Cosmopolitan magazine featuring Scarlett Johansson. .99 
LOOK AT HER: WOMEN IN WOMEN'S MAGAZINES

\section{List of Appendices}

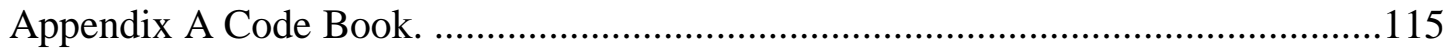

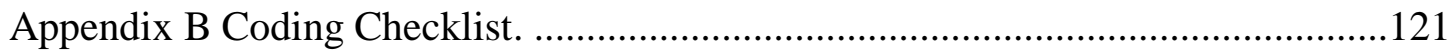


LOOK AT HER: WOMEN IN WOMEN'S MAGAZINES

"What is the greatest lesson a woman should learn?

That since day one. She's already had everything she needs within herself.

It's the world that convinced her she did not."

Rupi Kaur, 2015 


\section{LOOK AT HER: WOMEN IN WOMEN'S MAGAZINES}




\section{Chapter One: Introduction}

In 1993 the United Nations released its Declaration on the Elimination of Violence against Women in recognition of the pervasive neglect for women's rights to safety and security across the globe. Twenty-three years later, violence against women is still a major societal issue on a global scale (United Nations Women, 2016a). Physical and/or sexual violence is experienced by an estimated one in three women worldwide, typically within an intimate partnership (World Health Organisation, 2013). The pervasiveness of this phenomenon makes it an imperative field for research and analysis.

The first problem encountered in conducting sexual violence research is defining sexual violence (Kelly, 2013). Legal definitions tend to be narrow, heteronormative, and offer an intrinsically male perspective on what constitutes sexual violence (D'Cruze, 2012; Kelly, 2013). The New Zealand Crimes Act 1961, updated firstly in 1985 and then again in 2005, references sexual violence in two ways under the heading "sexual violation" (New Zealand Crimes Act 1961, s128). The first, rape, is defined as the penetration of one person's genitalia by another's penis, without consent. The second, unlawful sexual connection, is a broader term used to describe any non-consensual sexual connection between persons (New Zealand Crimes Act, 1961, s128). These definitions offer greater insight than they once did into the scope of sexual violence. However, only acknowledging "sexual violation" as a subheading within the larger continuum of sexual violence discounts a wide range of harmful behaviours. Sexual violence can also be defined as:

"Any sexual act, attempt to obtain a sexual act, unwanted sexual comments or advances, or acts to traffic, or otherwise directed, against a person's sexuality using coercion, by any person regardless of their relationship to the victim, in any setting, including but not limited to home and work."

World Health Organisation, 2002, p. 149

The broad scope of this definition makes it more suitable in the context of this thesis as I examine a nuanced potential source of ideas that support a culture where sexual violence, in both its overt and covert forms, can thrive.

An important acknowledgement to make at this point is that within the context of this thesis, when referring to sexual violence, reference is being made to violence against women by a male perpetrator. Sexual violence is not a phenomenon that is exclusively experienced by women, and other contexts in which sexual violence occurs are just as important to 


\section{LOOK AT HER: WOMEN IN WOMEN'S MAGAZINES}

critically examine. However, the current research focusses on the relationship between gender inequality and sexual violence and within inter-gender sexual violence the ratio of cases is skewed towards women being the victims of male perpetrators (Ministry of Justice, 2015).

Sexual violence has long been recognised as an extreme manifestation of gender inequality, an expression of male dominance irrevocably tied to entrenched historical power relations (World Health Organisation, 2002; Yodanis, 2004). In countries where the status of women is lower (as measured by to their representation in the workforce and higher education) it is more likely that men will use sexual violence against them (Yodanis, 2004). Interestingly, Yodanis's study found that this does not hold true for the use of physical violence against women. This suggests an explicit link between the status of women and the perpetration of sexual violence (Yodanis, 2004).

New Zealand has a history of strong feminist movements. In 1893, spurred by the resilient suffragette movement, it became the first country to grant women the right to vote in general elections (Cook, 2011). The second-wave feminist movement (late 1960s-1980s) also had significant successes. In 1960 and 1972, equal pay laws within the public and private sector came into force (Cook, 2011). Crucially, for sexual violence research, in 1985 the definition of rape was broadened within legislation, and in 1986 spousal immunity from rape charges was repealed (Barrington, 1986). Currently, New Zealand sits at ninth place out of 136 countries reviewed as part of the Global Gender Gap Report (World Economic Forum, 2016) implying that New Zealand as a nation continues to value and strive for gender equality. One would therefore be inclined to believe, based on the trends from Yodanis's (2004) research, that sexual violence against women would be relatively low. However, figures relating to sexual violence highlight an alarming prevalence. In the latest Crime and Safety Survey, conducted by the Ministry of Justice, it was reported that around one quarter of women experience at least one incident of sexual violence in their lifetime (Ministry of Justice, 2015). An interesting paradox, one that suggests that despite the successes to advance the status of women in New Zealand, sexual violence is still being used against them.

One factor that could be affecting the rates of sexual violence within New Zealand is the presence of rape culture. Rape culture is defined as a society where sexual violence is normalised, trivialised, and/or is an assumed inevitability (Buchwald, Fletcher \& Roth, 1995). There are an abundance of examples in recent history that trivialise the issue and/or perpetuate rape myths (falsehoods about sexual violence) thus contributing to rape culture 
within New Zealand (Bourke, 2007). Some memorable examples of rape culture in New Zealand from the last three years are detailed below.

In early 2016, Paula Bennett, a New Zealand Member of Parliament, wrote a post on her Facebook page advocating for the censorship of Wicked campervans who had come under fire for offensive slogans that objectified and encouraged sexual and physical violence against women (Kang, 2016). In response to her post, one comment stated "Bit of sexual violence never hurt anyone Paula. You [sic] should try a bit. Lol.” (Kang, 2016). There have also been instances where New Zealand public figures have expressed an attitude that reflects the idea that sexual violence is in some way a joke or a trivial issue. In November 2013, two radio hosts were criticised for their interview of a victim of the now infamous 'Roastbusters'. The Roastbusters were a group of young New Zealand men who set up parties with the intention of getting girls drunk and having sex with them, later uploading pictures and videos online. One of these girls was questioned by the radio hosts about why the girls were drinking in that situation and why they were out late at night before going on to describe the actions of the young men as "mischief” (Dougan, 2013).

In September 2016, following the alleged assault of a stripper at the Chiefs' (a New Zealand Super Rugby team) post season function, there was considerable criticism levelled at the alleged victim for putting herself in such a vulnerable situation. Margaret Comer, a representative of the team's major sponsor and a trustee on the board of the Waikato Women's Refuge, stated "if a woman takes her clothes off and walks around in a group of men, what are we supposed to do if one of them tries to touch her?" (Malone, Kerr, Hinon, Edens \& Robinson, 2016). Further to this incident, in January 2017, I personally observed a group of approximately 15 men, dressed in Chiefs uniforms, parading around the Wellington city with one man dressed in woman's clothing accompanying them. The fact that such an incident could be used as a parody costume for another rugby tournament speaks volumes about the attitudes towards allegations of sexual violence.

At the time of writing, more instances that highlight rape culture have occurred, this time spurring a protest by high school students. Senior pupils from Wellington College were revealed to have posted comments on a private Facebook page stating " $\mathrm{f} * * \mathrm{k}$ women" and "if you don't take advantage of a drunk girl, you're not a true WC [Wellington College] boy" (Williams, 2017). Young women from other high schools in the region planned a protest outside the college which was later moved to outside of Parliament when students from Wellington College "jokingly" threatened to run over any protesters at the school (Stewart, 2017). Further to this, in the same week, it was also reported that a group of Year 9 students 
(13 year olds) were suspended from St Patricks College in Wellington after they were caught making inappropriate videos and sexually harassing female staff members (Dooney, 2017).

These examples, aside from their demonstration of rape culture, are thematically similar in that to some extent the women involved in each incident have been sexually objectified. Sexual objectification, or sexualisation, refers to the deconstruction of a person in such a way that their sexual functions are separated from their actual identity (Calogero, Tantleff-Dunn, \& Thompson 2011; Smolak \& Murnen, 2011). It appears then that, despite the progress made by feminist movements and by women in New Zealand society, a trend exists where women are considered for their sexual worth above their achievements. The question is thus, how do these attitudes develop and how are they sustained?

Research has examined many arenas in search of an answer for this but the area of particular interest to me is the role of the media. The media is an agent of society; a platform to communicate en masse with people, and for this reason research has focussed on the effect it has on audiences. Media effects research is based on the idea that the 'masses' in society are vulnerable to behavioural conditioning from sources such as the media (Ericson, 1991; McQuail, 2000, in Jewkes, 2004; Reiner, 2007). While the relationship is not simply one of 'cause and effect', the media play a pivotal role in reinforcing and normalising ideas thus contributing to the impact of these ideas on their audiences (Burton, 2005; Ericson, 1991; Jewkes, 2004).

Past studies that have sought to link the media with rape culture have looked at various 'male-centric' media forms (such as pornography and men's magazines) and examined how these can influence opinions towards women and sexual violence (Krassas, Blauwkamp \& Wesselink, 2003; Milburn, Mather \& Conrad, 2000). Focussing on this type of media is undoubtedly an important area, however, an equally important area is the depiction of women in 'women-centric' media forms. If these media forms are suggesting that women should in some way expect sexual objectification it could be argued that sexualisation is therefore being normalised within the group it impacts. If this is the case the implications for change, progress, and equality could be profound.

Women's magazines are one such 'women-centric' media form. Diverse in topics, target audiences, and readership numbers, women's magazines all share a similar trait in that their role is to help define and reinforce ideas of what it means to be a woman (Burton, 2005; Croteau \& Hoynes, 2014; McCracken, 1993). This branch of the media was created to target the emergence of women as consumers and its enduring popularity and longevity suggests that this goal has been achieved. On the surface it may be construed as a positive that women 


\section{LOOK AT HER: WOMEN IN WOMEN'S MAGAZINES}

have their own corner of the media that is centred on the idea of communicating what it means to be a woman. However, analysis has shown that rather than being a haven for women to enjoy their womanhood in a space outside of the domain of men, this medium transfers ideas that exist in the patriarchal world, and repackages it as key information for women (Burton, 2005). Advertisements from women's magazines have been the subject of scrutiny in past content analyses for their depiction of traditional gender stereotypes as well as the sexualisation of women. Trends from these previous studies will be drawn on to inform the current research. However, this thesis will focus on the cover page of women's magazines. The cover page is what draws readers into the magazine, what sells them the idea that the publication can advise on how one can be the best woman they can be (McCracken, 1993). Therefore critically interpreting the ideas conveyed by these images is essential to furthering understanding about the lessons around femininity that may influence women.

The roots of this thesis sit firmly under the banner of sexual violence, gender inequality, and the role of the media in influencing, supporting, and reflecting societal ideas. By conducting an analysis of media content specifically targeted at women over a 40 year time period (1975-2015) it aims to capture any shifts linked to the changing status of women in society. The focus is on unravelling how women are depicted of particular interest is the sexualisation of women in images and the depiction of traditional gender stereotypes, because of the established link between these two features and sexual violence. Further to this, a wider discussion on how these media messages may influence women's ideas about themselves and others, and how this can impact attitudes towards and perceptions of sexual violence will be undertaken.

\section{Research Overview}

The objective of this thesis is to examine the cover pages of New Zealand women's magazines to investigate how women are depicted in a media form that is specifically tailored to a female audience. By using a content analysis this study set out to research these concepts with the following broad questions in mind:

1. How sexualised are the depictions of women on the cover pages of women's magazines?

2. Are traditional gender roles endorsed by the images on the cover of women's magazines? 
3. Has the material changed in regard to the level of sexual objectification and endorsement of traditional gender roles over a 40 year time period (19752015)?

This research is part of a larger Marsden-funded project that aims to critically examine the influence that three different media types may have on sexual violence within a New Zealand context. My own personal interest in the subject matter first piqued in 2013 after completing a university course on sexual violence. In hindsight it is fair to say I was naïve to be shocked as to how pervasive negative attitudes towards sexual violence are and how in this particular context gender inequality is still so pronounced. Eyes newly open, sexual violence and its ties to gender inequality seemed to be everywhere I turned. Sometimes there would be a step in a positive direction, such as the initial success of the UN Women's HeForShe campaign and the outpouring of support from both male and female celebrities (United Nations Women, 2016b). For the most part, though, incidents I observed were ones that conveyed damaging attitudes about women and sexual violence. This newfound sense of injustice on behalf of my gender was the motivation to engage with research which critically examines societal perceptions of women and sexual violence.

\section{Thesis Overview}

Having highlighted a broad overview of what this thesis aims to address in this first chapter, the following chapters expand on these ideas. Chapter Two provides a literature review which includes information about the four key areas that this thesis aims to address: sexual violence, the relationship between gender inequality and sexual violence, rape culture, and the role of the media in reinforcing these ideas. Chapter Three discusses the methodological approach for the current research including a detailed overview of the sample, coding schedule, reliability coding, and potential limitations of the research design. Chapter Four provides an overview of the quantitative results from the coding process, qualitative examples are used to illustrate the key differences found. Chapter Five discusses the results in a wider context and offers inferences about what the depiction of women on the cover pages of women's magazines may imply for gender inequality, rape culture, and sexual violence. In addition to this Chapter Five also details the potential applications of this research. Chapter Six concludes this thesis and discusses potential avenues for future research designed to delve further into the relationships between how women are depicted and their status in society. 


\section{Chapter Two: Literature Review}

This chapter entails a literature review that outlines the scope and prevalence of sexual violence within New Zealand, provides a historical backdrop to societal perceptions of this issue, and provides suggestions as to how this historical context influences our current culture. Further, it discusses the influence of the media on our perception of women, the connection between objectification, sexualisation, the depiction of gender stereotypes, and sexual violence within this society.

\section{Sexual Violence}

Globally, sexual violence is an under researched phenomenon. This contributes to the problem of establishing accurate prevalence rates. As discussed earlier, sexual violence has no universal definition that allows for consolidation of prevalence rates between studies, countries, or time periods. The most concerning barrier to assessing prevalence rates, however, is the research that estimates only $10 \%$ of women ever disclose their experiences of sexual violence (Mossman, Jordan, MacGibbon, Kingi \& Moore, 2009). Studies have shown that barriers to disclosure are varied and wide-ranging, but relatively stable across time (Sable, Danis, Mauzy \& Gallagher, 2006). Feelings of shame, guilt, and not wanting others to find out are reportedly the main concerns that dissuade disclosure. Other barriers include the fear of not being believed, concerns about confidentiality, and not acknowledging the incident as a sexual assault (Cohn, Zinzow, Resnick \& Kilpatrick, 2013; Jordan, 2012; Sable et al., 2006). Sable et al. (2006) acknowledge within their work that these reasons are the same as those given in studies conducted 30 years prior (before significant rape reform efforts were undertaken). The results then, from Cohn et al. (2013), who conducted their study within a similar population suggests that almost another decade has passed with little change to the barriers faced by victims of sexual violence. The issue of nondisclosure within the field of sexual violence is complex and this is a very brief summary of some of the reasons women feel unable to disclose their experiences. However, the pervasiveness of the reasons for nondisclosure suggests that changes in attitudes towards sexual violence on a broad societal scale have been difficult to attain. In addition, the very fact that women feel they cannot discuss their experiences of sexual violence is an example of society's failure toward victims of such crimes.

Globally, it is estimated that one in three women will experience some form of violence in their lifetime (World Health Organisation, 2013). Estimated prevalence rates vary 
from country to country, within the OECD nations estimated prevalence ranges from nearly one in two (47\%, in Mexico) to six in every hundred (6\%, in Canada) (Organisation for Economic Cooperation and Development, 2016). As a member of the OECD, New Zealand has the $5^{\text {th }}$ highest estimated prevalence rate (out of 33 countries) with data showing one in three $(33 \%)$ women will experience some form of violence in their lifetime (Organisation for Economic Cooperation and Development, 2016).

Estimated prevalence rates that specifically pertain to sexual violence also paint a grim picture for women in New Zealand. Abrahams et al. (2014) identified Australasia (New Zealand and Australia) as being a region with a high prevalence (16.4\%) for non-partner sexual violence - this is more than double the study's worldwide figure of $7.2 \%$ for nonpartner sexual violence. These percentages are based on an adult woman's lifetime risk of experiencing sexual violence in a non-partner context and are from a sample that includes 21 regions across Asia, Africa, Latin America, North America, Europe and the Pacific. This meta-analysis is not without its limitations but New Zealand based research and victim survey statistics also suggest a similar estimated prevalence rate.

Fanslow and Robinson (2004), for example, report non-partner sexual violence rates in populations from Auckland and North Waikato of 9\% and 12\% respectively. Prevalence rates for sexual violence within intimate partner relationships sit at a higher level of $14.1 \%$ in the Auckland region and $19.9 \%$ in North Waikato. In addition to this, figures from the New Zealand Crime and Safety victim surveys indicate that the lifetime prevalence for sexual victimisation is around $25 \%$ for women. The 2006 survey showed $28.3 \%$ of women had experienced at least one instance of sexual violence during the course of their life. In 2009 the figure was $24.8 \%$, and in the latest survey (2014) the figure was $23.8 \%$ (Ministry of Justice, 2007, 2010, 2015).

These estimates indicate that sexual violence in some form will be experienced by approximately one in four women in New Zealand. What is terrifying, though, is that research also tells us that nine out of ten women who experience sexual violence will never talk about their experiences of victimisation (Mossman et al., 2009) so the estimate of one in four women may actually be a drastic underrepresentation of the true figure.

\section{Gender Inequality and Sexual Violence}

Sexual violence is not a new experience or phenomenon; it is something that has been experienced by generations upon generations of women, and it has been perpetrated by generations upon generations of men (Jordan, 2001). This suggests that something within the 


\section{LOOK AT HER: WOMEN IN WOMEN'S MAGAZINES}

mechanics of society has enabled this pattern of violence to continue and, although it has changed form over time gender inequality is still recognised as an implicit factor in sexual violence (Jewkes, Flood \& Lang, 2015; Yodanis, 2004).

Examples of gender inequality are interwoven with the history of sexual violence; this is particularly evident if the framing of sexual violence in a legal context is examined. For instance, rape was initially considered as a type of property crime (D'Cruze, 2012). When a woman was perceived as little more than a domestic commodity, sexual violence against her was an affront to her father/brother/husband, or whoever could claim that his property was devalued by such an attack. Laws were in place to recompense father/brothers for lost value on a tradable asset, and to provide redress for husbands concerned with the potential impact on their lineage and social standing (Jordan, 2001). This notion of ownership is still an omnipresent part of some societies across the world. In New Zealand, the last legal remnant of this idea was not repealed until 1986; up until this point a woman was unable to successfully accuse her husband of rape as, sexually, she was still regarded as his property (Jordan, 2001).

In the $19^{\text {th }}$ century women were afforded some agency, in that they themselves were considered to be the victim rather than the recognition of harm being afforded to their male 'owner' (D’Cruze, 2012). However, the ubiquitous presence of patriarchal thought ensured the continued oppression of women victims of sexual violence. Definitions of sexual violence stated that the crime included any act that "all right-minded men, men of sound and wholesome feelings would say was indecent" (D’Cruze, 2012, p.34). This definition immediately discounted the experience of women and allowed men to set the terms for what was considered indecent behaviour. A further matter of importance to the men in power during the 1800s was the fear that rape accusations could be used against their gender as a tool of revenge. Rape was conceptualised as a crime that could only be perpetrated by a man against a woman. Therefore to ensure fairness to the accused (and to deny any power to the accusers), it was decreed that judges must advise jurors of the inherent danger of conviction based only on the testimony of the female victim (Gavey, 2005). Such suspicion still influences the small number of sexual violence cases that are actually reported to persons within the criminal justice system and undermines the very idea of a just legal system (Edwards \& Heenan, 1994). Further, these suspicions have also invaded public thought. In $1980,50 \%$ of those surveyed (men and women) believed that 'women lie about being raped' (Burt, 1980). This figure has lessened over time with only $22 \%$ of college-aged men agreeing that 'women lie about rape to get back at men' in a 2010 study (Edwards, Turchik, Dardia, 


\section{LOOK AT HER: WOMEN IN WOMEN'S MAGAZINES}

Reynolds \& Gidycz, 2011). Regardless of the decrease, the precedent set by these legal statutes for perceptions within and outside of the criminal justice system has been profoundly detrimental.

Another suspicion that has plagued victims of sexual violence is the unremitting fixation on the moral decency of the woman involved. The notion of appropriate femininity (the adherence to traditional feminine gender roles) has served as a tool to undermine women's testimonies and is still to this day a determining factor in the outcome of sexual violence cases (Edwards \& Heenan, 1994; Gavey, 2005). Women who did not conform to the preconceived ideas of 'respectability' or 'femininity' were often implicated in their own rapes. Their characters were demeaned, their morals questioned, and often their accusations were judged as an effort to preserve their façade of moral respectability. Rape was labelled as a woman's 'indiscretion' and her accusations were deemed reactions to the overwhelming feelings of regret, shame, or fear following this imprudence (D'Cruze, 2012; Edwards \& Heenan, 1994; Gavey, 2005).

The social status of women has determined thought around sexual violence throughout history. The treatment of female victims of sexual violence is an outcome of the socially constructed gender binary where masculine traits and feminine traits are diametrically opposed. This division reinforces the idea that one gender is superior to the other and this notion of superiority facilitates violence against the oppressed group (Jewkes, 2002). Society dictates what is and is not acceptable for both men and women (Jackson and Scott, 2002). For every action that teaches boys that they must be strong, stoic, adventurous, and dominant, there is an opposing action that teaches girls to be soft, emotionally open, reserved, and submissive (Kachel, Steffens \& Niedlich, 2016). These lessons reinforce a polarising divide between genders and facilitate a hierarchy in which men are more advantageously positioned than women. So when, in 2013, the 'Roastbusters' scandal (detailed earlier) caused some to question "how has New Zealand raised such sons?" (Trotter, 2013), the answer was relatively simple. It is how we have always raised sons. The male sense of privilege has been and continues to be the cornerstone of gender based violence as it enables men to view women as lesser beings (Vance, Sutter, Perrin \& Heesacker, 2015).

The historical examples highlighted above are just the tip of the iceberg when it comes to the oppression of women victims of sexual violence by patriarchal structures. This said, it is important to acknowledge where progress has been made. Thanks largely to the second-wave feminist movement (late 1960s-1980s) the profound negative effects of the male-centric perspective on sexual violence cases within the judicial system were exposed 
(Edwards \& Heenan, 1994; McDonald, 2014). Legislation now exists that is designed to protect women from judgment by gender 'norms' during the course of rape trials. This includes the proviso that a woman's sexual history cannot be questioned, or even referred to, during the course of the trial, unless it is specifically and substantially relevant to the case at hand (Evidence Act 2006, s44). Also, the mandatory warning about the victim's testimony that judges were once required to give is now something that is done at the judge's discretion (Edwards \& Heenan, 1994; McDonald, 2014).

Outside of the legal system, support for victims and acknowledgement of sexual violence as a serious issue has also progressed. There has been a significant increase in public awareness of sexual violence with the shifting of the issue from a 'private' matter to one of interest to public health (Carmody \& Carrington, 2000). A recent example of this has been the New Zealand-wide, student-led, Thursdays in Black movement. This movement encourages students to wear black every Thursday to increase visibility and show solidarity with survivors of sexual violence (Thursdays in Black, 2016). The emergence of specialist support services that provide victim-centred care with the aim of reducing harm after sexual violence was another victory for second-wave feminism (Jordan, 2001). However, increased state funding and recognition is still required for these services to achieve their full potential and to reach all victims of sexual violence (Ministry of Social Development, 2017).

Despite the notable progress made by the second-wave feminist movement, it is not the case that feminism has abolished gender inequality (Kinser, 2004). Although, the last 40 years have been a period of significant positive change equality is an illusion. Societal expressions of sexism have become more covert and an ethos of acceptance regarding the sexual victimisation of women is sustained through rape culture, sexual objectification, and the pervasiveness of traditional gender stereotypes. Each of these phenomena will be examined in detail in the following sections of this chapter.

\section{Rape Myths and Rape Culture}

\section{Rape Myths}

Rape myths are a series of falsehoods about sexual violence that are widely held and endorsed within society (Ryan, 2011). They are the by-product of a history of gender inequality and rigid traditional gender norms, and serve to reinforce historic notions of women victims of sexual violence (Bourke, 2007; Shapcott, 1988). The most prevalent myths about rape have ties to historical beliefs about sexual violence and the role of women in society (Edwards et al., 2011). There are a range of rape myths that exist, three of which are 
examined in this section. The idea that 'women cannot be raped by their husbands', 'women enjoy rape', and 'women ask to be raped' are beliefs that have existed in public consciousness across time and have ties to the historical status of women in patriarchal societies (Burt, 1980; Edwards et al., 2011; Ryan, 2011; Shapcott, 1988).

The first of these false assumptions, 'women cannot be raped by their husbands', is directly tied to the historical role of women as domestic commodities for their husbands. We see this idea framed within legislation, with many legal codes endorsing the idea that marriage was the ultimate symbol of consent and husbands had every right to sexual contact with their wives whenever they desired (Edwards et al., 2011). In New Zealand, women were not able to bring a case of rape against their husbands until 1986 when this idea was specifically addressed and repealed within New Zealand statutes (Jordan, 2001). The notion that men can still claim some form of sexual entitlement towards their partners is deeply rooted in the historical perspective of male superiority over women (Jewkes, 2002). This mentality relegates women to sexual objects there solely for the pleasure of the man who can claim 'ownership'.

The idea that 'women enjoy rape' has two points of origin. The first stems from the belief that if a woman truly does not want to be raped she will be able to physically resist the attack. Physical resistance will either be successful or the woman will sustain significant physical injury which renders her unable to continue to resist the attack, thus enabling her attacker to sexually assault her (Edwards et al., 2011). An infamous demonstration of this belief is the use of the 'Coke bottle and the pencil' where defence lawyers demonstrated that if a juror tried to put a pencil in a Coke bottle it could not be inserted if the bottle kept moving (Jordan, 2001). The Coke bottle represents a woman who is truly resisting rape and 'proves' that rape is preventable as long as there is active resistance. This 'metaphor' is another example of the male perspective being forced onto women's experiences because men are taught that it is acceptable to defend themselves whereas women are not (Dietz, 1998). Masculinity allows for strength, aggressiveness and dominance and these lessons reinforce for men that it is acceptable to resort to force if they are challenged. The socialisation of women and traditional femininity, however, teaches passive or avoidant responses to challenge or conflict (Dietz, 1998). How is it then reasonable to expect women to utilise their 'fight' instinct over 'flight' or 'freeze' when they have been taught that the latter are the expected and appropriate ways for them to respond?

The other source of the 'women enjoy rape' myth stems from the historical school of thought known as 'sexology'. This ideology posited that women's sexuality manifests as 


\section{LOOK AT HER: WOMEN IN WOMEN'S MAGAZINES}

coyness but instinctively they desire to be dominated and violated by men (Gavey, 2005). The creation of this sexual script is tied to traditional gender norms where men are expected to be the inciters of sexual behaviour and women to passively accept them. Removing the legitimacy of resistance or disinterest from women within sexual scripts sets a dangerous precedent for women in society. If all resistance from women is framed as part of the "game of courtship" (Gavey, 2005, p. 20), sexual aggression towards women is encouraged and normalised.

The idea that 'women ask to be raped' echoes sentiments that the extent to which women adhere to appropriate femininity somehow influences the occurrence of sexual violence (Edwards et al., 2011). The further female victims of sexual violence deviate from traditional feminine behaviour, the more blame is placed on them for said violence (Fraser, 2015). Invalidating experiences of sexual violence because of a victim's clothing, behaviour, or sexual history explicitly suggests that women who are not traditionally feminine are undeserving of support, justice, or recognition. This attitude furthers oppression because societal protections are afforded to those who fit in the mould society has created for them, while those who do not are threatened with rejection and hostility (Fraser, 2015).

Rape myth acceptance is prevalent across various populations within Western society and is instrumental in maintaining a climate that fails to support victims of sexual violence (Burt, 1980; Edwards et al., 2011; Lonsway \& Fitzgerald, 1994; Shapcott, 1988). What is particularly concerning is the lack of real change in contravening these beliefs, with literature from the 1980s showing the same patterns as literature from 2015. One of the older sources, and one of the first texts on rape myths published within a New Zealand context suggests that "institutions tend to embody the perspective that is acceptable to the patriarchal power structure" (Shapcott, 1988, p.198). This assertion, in tandem with the lack of diversity across time in these beliefs, suggests that similar power structures are still in place.

\section{Rape Culture}

Further evidence towards the idea that patriarchal thought still influences public opinion on sexual violence is the presence of rape culture in New Zealand. Rape culture, as defined in the previous chapter, refers to societies that tend to normalise, belittle or assume sexual violence is somewhat inevitable (Buchwald, et al., 1993). Rape myths help to facilitate the development of this cultural mind-set, but other derivatives of gender inequality, such as the sexual objectification of women, also help to create a climate in which sexual violence against women is facilitated. 
"[Rape is] nothing more or less than a conscious process of intimidation by which all men keep all women in a state of fear."

Brownmiller, 1975, p.15.

In the forty-two years since this quote was published, it has caused controversy and debate within and between societal groups (Bohner \& Schwarz, 1996). However, if 'rape' is considered in the sense of rape culture rather than the literal act, this is what occurs and is occurring now. Rape culture safeguards sexual violence against women - perpetrators are routinely protected and victims are routinely silenced (Johnson, 2014).

Rape culture is the mechanism at work when a man can describe kissing women and "grab[bing] them by the pussy" without their consent, explain it as "locker room talk... It's just words, folks", and still be elected President of the United States ${ }^{1}$ (Mahdawi, 2016). Rape culture enables a lawyer to successfully argue that an incident of rape was "reluctant consent" and about "a young man who thought he could... There's nothing horrible about that, it's just a reflection of life",2 (Feek, 2016). When New Zealand Rugby is more concerned with the image and reputation of a club than a woman's allegations of harassment and assault by players - that's rape culture ${ }^{3}$ (Sachdeva, 2016). Outside of specific incidents of sexual violence (which all the above examples describe) rape culture is also present. Hit songs that describe "[hating] these blurred lines" when it comes to a woman's consent ${ }^{4}$ (Lynskey, 2013), or schools that suspend teenage girls for wearing shorts or exposing their shoulders because their bodies are a distraction for male students and staff ${ }^{5}$ (Bates, 2015) are also facilitating rape culture.

${ }^{1}$ Comments made by President Donald Trump on camera to Billy Bush in an Access Hollywood segment in 2005. The tape was released in October 2016 during the final stages of the 2016 US Presidential Election campaign.

${ }^{2}$ Comments made by Philip Morgan QC in defence of his client, Scott Kuggeleijn. A young woman alleged that she had been raped the morning after inviting the defendant home with her. She had refused sexual intercourse the night before and alleged that the next morning she was pinned down as Kuggeleijn had intercourse with her against her will. The original trial resulted in a hung jury, then in February 2017 Kuggeleijn was found not guilty.

${ }^{3}$ In August 2016, players from New Zealand rugby team, the Chiefs, hired a stripper as part of their post-season celebrations. The woman, 'Scarlette', later alleged that players had touched her forcefully without her consent, physically intimidated her, and threw gravel and alcohol at her during the course of the evening. New Zealand Rugby conducted a widely criticised internal investigation and found no evidence to support the allegations. The organisation issued a public apology for hiring the stripper and refused to apologise for the other behaviours.

${ }^{4}$ These lyrics are part of the single "Blurred Lines" written by American artists Robin Thicke, T.I., and Pharrell Williams. This song was number one in 25 countries, including New Zealand.

${ }^{5}$ In the United States in particular there is growing outrage amongst high school students of the 'fairness' of being “dress-coded". Female students in particular are expressing frustration over being sent out of class for wearing clothes that may 'distract' male students from learning or even more disturbingly male teachers from teaching. 
Rape culture is omnipresent yet there is still debate as to its existence. In New Zealand, there has just been a protest on rape culture in response to an incident involving boys from Wellington College ${ }^{6}$ (Williams, 2017). Online news sites were flooded with comments and divisive opinion pieces. One comment struck what I think the heart of the issue is:

“...it would be evidence of rape culture if there were any adult support for what these boys said... it's an injustice to the school and to the great majority of boys in it."

Thorpe, 2017

Whilst the writer of this piece goes on to rather ironically lament that he is not arguing that 'boys will be boys', the above sentiment screams of that mentality. Framing examples of rape culture as 'one-offs' just serves to further trivialise the issue. How many 'one-offs' of this thematically identical behaviour are needed before the existence of rape culture is acknowledged? Before recognition of the trivialisation of sexual violence against women is truly framed as what it is - the continuation of the mind-set that women are inferior to men.

\section{Rape Culture and the Media}

The pervasive nature of rape culture is reinforced by various branches of the media (Finley, 2016; Greer, 2007). Criticisms have been cast on both news and fictional media (Greer, 2007). News media have been found to contribute to rape culture with their selective reporting of rape cases, their tendency to discount women's experiences, and their minimisation of the seriousness of offences (Greer, 2007). Coverage of sexual violence also tends to categorise women as 'virgins' or 'whores'. This grouping perpetuates the myth that 'women ask to be raped' and reinforces the assumption that if women adhere to traditional femininity they will be safe from sexual violence (Greer, 2007). Fictional media have also been criticised for their role in normalising sexual violence against women (Finley, 2016). One such example is the controversial video game, Grand Theft Auto V. This game allows players to purchase women who perform sexual acts on their avatar. Players are then encouraged to murder the female character in order to get their money back (Saada Saar, 2014). This harmful 'norm' construction in widely accepted and available popular culture

\footnotetext{
${ }^{6}$ In March 2017, senior pupils from Wellington College were revealed to have posted comments on a private Facebook page stating " $\mathrm{f} * \mathrm{k}$ women" and "if you don't take advantage of a drunk girl, you're not a true Wc [Wellington College] boy" inciting anger and debate about rape culture nationwide.
} 


\section{LOOK AT HER: WOMEN IN WOMEN'S MAGAZINES}

exemplifies rape culture as the status of women is demeaned and sexual violence is trivialised.

Previous literature has examined the potential effect of media images on the perceptions of women, and of sexual violence in various media types (Krassas et al., 2003; Milburn et al., 2000). These studies have tended to focus on aspects of the media that typically cater for a male audience. For example, Milburn et al. (2000) investigated the effects of viewing objectified women in R-rated cartoon films. The authors predicted that sexually objectifying images would be linked to higher endorsement of rape myths, gender stereotypes, and victim blaming. The results suggested that sexually objectifying images of women influences the perception of rape. Namely, participants who viewed the objectifying media were more likely to view an acquaintance rape scenario ${ }^{7}$ as consensual and something the victim 'wanted' (Milburn et al., 2000). Notably, the authors in this study made a point to use sexually objectifying images that, whilst R-rated, were not classed as pornographic (in that they did not explicitly contain sexual intercourse), to exemplify that images considered to be mainstream can have these effects.

In addition to this, Krassas et al. (2003) also examined the presence of objectifying images in gendered media spaces. The research examined magazine advertisements focussing on the presence of sexist ideology in images of women within men's magazines. It was found that in this media form, women were more likely than men to be depicted in sexually objectifying ways. The authors suggest that the clear commodification of women could reinforce gender differences and negative ideas about sexuality (Krassas et al., 2003).

Research that has focussed on the depiction of women in women's media has examined the creation of the 'ideal woman' (McCracken, 1993). Advertisements (a term which could arguably encompass magazine cover pages) are designed to present an idealised vision of life, designed to make viewers connect acquisition of a product to attaining that ideal (Richins, 1991). In this context, the selection of women to use in images is invariably tied to this idea of the 'ideal'. Choosing to almost exclusively present one "type" of woman reinforces this message, and thus the 'ideal' woman who is white, thin, young, and classically beautiful is created (Haboush, Warren \& Benuto, 2012). In creating an 'ideal' advertisers and publishers set unrealistic standards that although viewers can, in most cases identify as unrealistic, are still a used as a comparison point by women (Richins, 1991). Women comparing themselves to an unattainable 'ideal' on attributes solely relating to appearance

\footnotetext{
${ }^{7}$ Acquaintance rape refers to sexual violence committed by a person already known to the victim.
} 


\section{LOOK AT HER: WOMEN IN WOMEN'S MAGAZINES}

creates a situation in which three key areas that may also have a role in reinforcing rape culture, objectification, sexualisation, and gender stereotypes, can thrive.

This research focusses on media images of women (specifically images on the covers of women's magazines) and the impact that these may have on audiences. Images are more than just pictures, they have value, meaning, and can reflect prevailing societal ideas (Burton, 2005; Carrabine, 2012). Whilst there will always be an individual element to the connection people form with images they look at, those in power will seek to influence audiences with the images selected for publication (Croteau \& Hoynes, 2014; Sonnevend, 2012; Watt, 2012). Images presented to viewers are not chosen randomly, they are there to attract and entice consumers. As such there is a reluctance to present visual information that deviates from the stereotypical norm in any given context (Griffin, 2010; Schwalbe \& Dougherty, 2015; Sonnevend, 2012). Images in publications can therefore be analysed and interpreted as messages to audiences.

\section{Objectification}

Objectification refers to the conceptualisation of people as things. Objectification is deemed present in images where a person is there for the pleasure/consumption of the viewer (Fredrickson \& Roberts, 1997). It is more common for women than men to be depicted as such because of heterosexual power dynamics (Calogero et al., 2011). Women have long been defined by their relationships with and to men, and objectification is another manifestation of this. Objectification theory is a framework that aims to explain how women's bodies are viewed in various sociocultural contexts and the impact that objectifying images have on female viewers (Fredrickson \& Roberts, 1997). Studies have shown that women and young girls who are exposed to objectifying stimuli are socialised to expect being treated as objects and also begin to view themselves through this lens (Calogero et al., 2011). The internalisation of these messages is known as self-objectification and it is characterised by persistent scrutiny of one's body which impacts the perception of oneself and others (Calogero et al., 2011; Fredrickson \& Roberts, 1997).

Fredrickson, Roberts, Noll, Quinn and Twenge (1998), for example, found that women with a higher Body Mass Index (BMI; a rudimentary measure of health based on height and weight) had increased feelings of body shame and that variance in this was predicted by self-objectification. The study manipulated self-objectification between groups by having one group of women try on a sweater and fill out questionnaires on body shame, whilst the comparison group tried on a swimsuit and completed the same survey. The 


\section{LOOK AT HER: WOMEN IN WOMEN'S MAGAZINES}

relationship between high BMIs and body shame was amplified in the group who were asked to view themselves in a swimsuit rather than in a sweater. The findings of this study suggest that women have an internalised image of the 'ideal' and those who do not fit into this criteria are more critical of themselves (Frederickson et al, 1998).

Other studies have noted the negative impact of various forms of mass media that inspire feelings of self-objectification have in women, including eating disorders, depression, and sexual dysfunction (Aubrey, 2007; Fox, Ralston, Cooper \& Jones, 2015; Fredrickson et al., 1998; Halliwell, Malson \& Tischner, 2011; Morry \& Staska, 2001). Self-objectification has also been linked to increased levels of self-harm in college-aged women (Muehlenkamp, Swanson \& Brausch, 2005).

\section{Sexualisation}

Sexualisation is a subsection of objectification. It goes beyond viewing a person as a commodity to framing an individual in such a way that just their sexual functions are used to represent them as a whole (Calogero et al., 2011; Smolak \& Murnen, 2011). Sexual objectification in images is often driven by the male gaze, where women are depicted for the pleasure of men (McCracken, 1993). Sexualisation is seen as another tool of oppression as objectifying views can be internalised and thus play a role in the replication and indoctrination of gender norms (Smolak \& Murnen, 2011; Szymanski, Moffitt \& Carr, 2011). Sexualisation is driven by cultural norms and has come to be something that most women will experience either overtly (such as sexual comments, harassment, or in extreme cases experiences of sexual violence) or covertly, typically through different forms of media (Calogero et al., 2011; Szymanski et al., 2011). This makes sexual objectification a key point of interest in this thesis as if it is a feature of images in a media form specifically catered for women it may have a role in influencing perceptions of their own gender.

Past studies that have examined the content of women's magazines have found that the images of women both on the cover pages and from deeper within the publication reinforce gender norms and sexualisation which could contribute to the subordinate position of women in society (Hatton \& Trautner, 2011; Reichert, Lambiase, Morgan, Carstarphan \& Zavoina, 1999; Soley \& Kurzbad, 1986).

Soley and Kurzbad (1986) conducted a comparative analysis of advertisements from 1964 and 1984 within a range of general interest, women's and men's magazines. Their findings indicated that in both men's and women's magazines the proportion of sexualised adverts remained stable (around one quarter of all advertisements) but the overall number had 


\section{LOOK AT HER: WOMEN IN WOMEN'S MAGAZINES}

increased dramatically. In general interest magazines both the proportion and overall number had increased significantly. These findings indicate that sexualised portrayals of women within women's magazines have been relatively commonplace for decades (Soley \& Kurzbad, 1986).

Similarly, a study by Reichert et al. (1999) indicated that when compared to advertising images from 1983, images of women from 1993 were more sexually explicit. This study looked at all large scale advertisements within publications and as well as coding for sexual explicitness overall, split the data into audience groups (general interest, women's, and men's) for comparison. This suggests that the use of sexualisation may be increasing across time. These analyses showed the same pattern as Soley and Kurzbad (1986) whereby advertisements within general interest magazines were less sexually explicit compared to gendered publications.

Hatton and Trautner (2011) did not specifically analyse women's magazines, instead doing a comparative longitudinal study of the sexualisation of men and women on the cover pages of general interest magazine, Rolling Stone. This research found that women were more likely to be sexualised than men across the entire time period studied (1960s-2000s). In addition to this the authors found that for images of women the level of sexualisation increased significantly over the researched time period. Images from the 2000s had the highest level of hypersexualised images (images that scored over 10 on their coding schedule that had a maximum score of 23). Whilst not within the niche medium of women's magazines, these results show a similar trend of increases in sexualisation to the work of Reichert et al. (1999). Interestingly, Hatton and Trautner (2011) focussed on a general interest magazine which both Soley and Kurzbad (1986) and Reichert et al. (1999) found to be less sexualised than gendered publications. If the above pattern has held true, it could be predicted that depictions of women in women's magazines from the 2005 and 2015 time periods within this research will also be hypersexualised. This is an alarming hypothesis because the negative impact of sexualised depictions of women in women's magazines has been established by many studies (Aubrey, 2007; Aubrey, 2010; Morry \& Staska, 2001). Morry and Staska (2001), for example, found that reading beauty magazines led to the internalisation of societal ideals (such as 'thin is the ideal form for women') and selfobjectification. This result was replicated by Aubrey (2007) who identified a positive correlation between exposures to sexually objectifying magazines (and television) and body shame. A further correlation between exposure to sexualised images and an increased level of self-objectification was also found. In a later study, Aubrey (2010) found that messages that 


\section{LOOK AT HER: WOMEN IN WOMEN'S MAGAZINES}

could influence self-objectification were found in $40 \%$ of captions in health magazines for women. The study defined objectifying phrases as a specific mention of wanting/getting/changing a body part to make it more desirable (Aubrey, 2010).

Sexualisation, like objectification, can be internalised and have similar negative effects to self-objectification on women. Halliwell et al. (2011) found that magazine advertisements featuring provocative images (scantily clad women) were strongly linked to higher levels of self-objectification. This result was especially pronounced for advertisements with captions that emphasised the woman's sexual agency, which is troubling given the increasing trend for this within advertisements (Halliwell et al., 2011). In addition to creating and reinforcing negative mental states and self-opinion, self-sexual objectification can also impact behaviour. As described earlier, Fredrickson et al. (1998) in a secondary experiment found that women in the swimsuit condition performed worse on a rudimentary maths test than those in the sweater condition. When controlling for previous mathematical performance the relationship was still significant. This led to a tentative conclusion that self-sexual objectification may impact the attentional capacity of women which may then lead to poorer performance on academic tasks (Fredrickson et al., 1998).

Exposure to sexualised portrayals of their gender not only influences negative selfthought in women but can also affect their view of other women. One study, for example, showed how sexualised images can have a profound effect on challenging the oppression of women. Calogero (2013) found that higher levels of self-objectification correlated with higher levels of justification for stereotypical gender roles and were also predictive of lower levels of gender-based activism in a sample of college women from the United States. A secondary experiment manipulated self-objectification by asking one group of participants to remember a time where they themselves had been objectified. After being asked to think about a personal instance of sexual objectification, women were less willing to participate in activism that would challenge stereotypical gender roles (Calegero, 2013). This suggests that sexualisation may contribute to silencing women on issues of gender inequality.

Furthermore, research has shown that self-objectification can also have an impact on women's perceptions of sexual violence. Fox et al. (2014), for example, conducted a study and found (in another sample of American college-aged women) repeated exposure to sexualised avatars predicted higher levels of self-objectification. Higher levels of selfobjectification in turn predicted greater acceptance of rape myths (false beliefs about sexual violence). These results suggest that sexualised depictions of women within media forms have a lasting impact on perceptions and attitudes towards rape culture (Fox et al., 2014). The 


\section{LOOK AT HER: WOMEN IN WOMEN'S MAGAZINES}

relationship between rape myth acceptance and self-sexualisation highlights that objectification can lead to the dehumanisation of certain groups which enables the justification of violence against them (Vance, et al., 2015).

A further example of the dehumanising effect of sexualisation can be seen in the results of a study by Loughnan, Pina, Vasquez and Puvia (2013). These authors examined how an objectifying image can change the perception of rape victims. In their study participants were shown an image of a woman they called 'Laura' who was either nonsexually objectified (wearing jeans and a t-shirt) or an image of 'Laura' wearing a bikini (sexually objectified). They then had to read a short description of a scenario in which 'Laura' was raped by 'Mike' whom she had agreed to go on a date with after meeting him at a party. Participants were then surveyed on measures of victim blame, perceived suffering, and moral concern for the victim. Those who had been shown the objectifying image were found to attribute more blame to 'Laura', their moral concern for her was diminished, and she was perceived to have suffered less.

In sum, past research has established that a pattern of sexualisation exists in women's magazines (Hatton \& Trautner, 2011; Reichert, et al., 1999; Soley \& Kurzbad, 1986). Media images of women normalise the idea that the fairer sex is there for the viewing pleasure of men (Calogero et al., 2011; Smolak \& Murnen, 2011; Szymanski et al., 2011). These images of women also serve to oppress women by diminishing their self-worth and facilitating a divisive rhetoric within the gender (Aubrey, 2007; Fox, et al., 2014; Fredrickson, et al., 1998; Halliwell, et al., 2011; Loughnan, et al., 2013; Morry \& Staska, 2001; Muehlenkamp, et al., 2005).

\section{Gender Roles}

The third key area of interest is the depiction of traditional gender roles in images of women. As discussed in previous sections, gender inequality, specifically the dominant position of men in society, is thought to be a causal factor in the appalling rates of sexual violence towards women (Yodanis, 2004). Gender inequality is reinforced by notions of gender norms that, in the simplest terms, convey the idea that men are dominant and women are submissive (Kachel et al., 2016). Assessing the prevalence of these messages in women's magazines, a medium that shapes the notion of what it means to be a woman, is important to understand how this could be affecting the reinforcement of a harmful societal norm.

Erving Goffman's 1979 work, Gender Advertisements, is considered an influential text in the analysis of gender within images. Goffman (1979) provides a framework for the 


\section{LOOK AT HER: WOMEN IN WOMEN'S MAGAZINES}

ways that traditional gender roles are conveyed in images and argues that the reinforcement of such stereotypes contributes to a continuing gender imbalance. Content analyses suggest that traditional gender norms are present in women's magazines and have been consistently since the earliest studies (inspired by the second-wave feminist movement in the early 1970s) investigated this phenomenon (Lindner, 2004). Sullivan and O'Connor (1988) conducted a comparative analysis of advertisements from the 1950s, 1970s, and 1983. This study examined the depiction of women within the workforce and it was found that advertisements from 1983 were more likely to show women as independent and in a range of careers. However, during the same time period, women were increasingly likely to be depicted as 'ornaments' or in sexualised roles. The authors inferred from these results that the depiction of gender roles has transformed rather than lessened which counterbalances progress towards gender equality (Sullivan \& O'Connor, 1988).

Similarly, more recent studies that utilised Goffman's framework have found few changes in the portrayal of gender stereotypes in images of women (Kang, 1997; Lindner, 2004). Kang (1997) examined advertisements in women's magazines for the presence of traditional gender roles across the time period 1979-1991. The results showed very few changes in the overall depiction of stereotypical gender roles. However, Kang (1997) did find a similar change to that of Sullivan and O'Connor (1988) in the how to the way gender roles had changed in their depiction. Namely, the more modern advertisements were less likely to feature overt depictions of masculine dominance (where men were larger than women in images or where men had control over women in images) and more likely to show women as sexualised or lacking agency within images. Lindner (2004) examined magazine advertisements from 1955 to 2002 in order to examine patterns in the depiction of gender roles across that time period. Concurring with the results from Sullivan and O'Connor (1988) and Kang (1997), results showed few changes across the time period and reinforced that stereotypical images were common within advertisements (Lindner, 2004). Lindner (2004) also compared advertisements between a general interest magazine (Time) and a woman's publication (Vogue). Interestingly, the results showed that more overt depictions of gender roles were significantly more prevalent in Time than in Vogue but in all other categories advertisements from Vogue were significantly more stereotypical than those in Time. This result again reinforces the idea that the presence of stereotypical gender roles is ubiquitous within magazines but its form is fluid (Lindner, 2004). 


\section{Summary}

Sexual violence is irrevocably linked to gender inequality and the dehumanisation of women (Johnson, 2014; Vance, et al., 2015). The normalisation of sexual objectification and the reinforcement of traditional gender stereotypes helps to sustain a damaging and pervasive rape culture and ultimately contributes to gender inequality. Images can reflect norms that societies want to create and sustain (Burton, 2005; Carrabine, 2012; Sonnevend, 2012) and media images of women reflect patriarchal ideals that are, on the whole, damaging to the gender (Aubrey, 2007; Calogero, 2013; Fox, et al., 2014; Fredrickson, et al., 1998; Halliwell, et al., 2011; Loughnan, et al., 2013; Morry \& Staska, 2001; Muehlenkamp, et al., 2005). By examining images of women in media aimed at women this thesis aims to investigate, within a New Zealand context, the prevalence, patterns, and power images in a specific media form - women's magazines - have in reinforcing gender inequality and a culture supportive of sexual violence against women.

\section{The Research}

Women's magazines cater for a diverse age range and harbour a wide range of niche foci, but share one overarching theme - to define and uphold the idea of what it means to be a woman (Burton, 2005; Croteau \& Hoynes, 2014). A simple interpretation may make this seem advantageous for women, that this corner of the media is for women to be women, free from the interests of men. However, this genre of media is designed to take advantage of the emergence of women as consumers (Burton, 2005). The cornering of this new market has meant many of the traditional practices have carried over, and women's magazines offer an escape into the life of the 'ideal' woman. The cover page is therefore key because without an enticing cover the magazine cannot hook consumers (McCracken, 1993). The cover page must be alluring to its target audience, and it must set the tone for what is to follow within the pages of the publication (McCracken, 1993). This part of a publication has a crucial role and is carefully designed which allows for the interpretation of deeper messages for an exclusively female audience (Sonnevend, 2012). It is for these reasons that the cover page is the focus of this thesis.

The evidence of the construction of harmful normative standards within women's media make it all the more important to investigate in different contexts. My research focusses on one specific type of media image (magazine cover page photos) and examines depictions of women within a space designed exclusively for women. This study aims to address how stereotypical and sexualised depictions of women create a harmful 'norm' that 
contributes to the perpetration of rape culture and can lead to negative outcomes for women and society. By analysing magazines across a 40 year time period I will also investigate how the mechanisms of norm-creation within the media may have changed over time. Based on the results of previous literature, the hypotheses that will guide the analysis are as follows:

\section{Sexualisation}

1. I predict that women will be depicted in a sexualised manner within cover images;

2. I also hypothesis that the level of sexualisation will have increased across the timespan (1975-2015).

\section{Gender Roles}

1. I predict that images of women on cover pages will be depicted in a way that is consistent with traditional gender stereotypes;

2. In addition, I predict that there will be little overall change in gender role 'scores' across time;

3. Further, I hypothesise that where women are shown in stereotypical ways it will be more likely that this is through the removal of agency in images rather than the overt positioning of a dominant man within the image.

The next chapter will give an overview of the methodology used in the current study. The development of the coding schedule as well as a detailed overview of the coding categories and potential limitations of the research design are discussed. 


\section{Chapter Three: Methodology}

\section{Overview}

The overarching aim of this research project is to examine the visual depiction of women, in women's magazines, from 1975 until 2015. Undertaking a longitudinal examination of cover pages from New Zealand women's magazines will allow for the identification and study of patterns of sexualisation and objectification over time. Cover pages, were chosen as the focus of this study as the cover page of a magazine represents carefully selected images designed to appeal to the target audience and bolster sales. The conscious selection of these images for an audience of women enables the examination of the intended messages from the media to women (the primary audience for the magazines in this study). Under particular scrutiny is the depiction of women in regard to the level of sexualisation and the portrayal of gender roles in the images on the cover pages. The project contributes to a larger, Marsden-funded study which aims to analyse and discuss the portrayal of women across multiple media types.

From the outset of the project it was determined that content analysis would be the most appropriate way to explore this topic as it is a way of quantitatively examining patterns and messages that exist within different media forms (Hsieh \& Shannon, 2005). Hsieh and Shannon (2005) describe three main branches of this methodology: conventional, directed, and summative content analysis. Conventional content analysis is primarily used where the existing research base on a phenomenon is limited. Researchers approach content without specific coding categories, noting down key features as the material is analysed. Coding categories are thus shaped from the material. Directed content analysis is used where a large literature base can contribute towards the knowledge of the material prior to the coding phase. This allows for coding categories related to a certain phenomenon to be developed for the material as key features are already known to the researcher. Summative content analysis aims to explore the usage of certain features within material - this can be as a count of the use of a certain word or image device and is typically utilised to study the context in which certain features are used (Hseih \& Shannon, 2005).

There are several theoretical areas and previous research studies that informed the direction of my analysis. The most appropriate research technique was therefore a directed content analysis as the features of interest could be developed into coding categories prior to the commencement of the analysis. The process that produced the finalised coding scheme 


\section{LOOK AT HER: WOMEN IN WOMEN'S MAGAZINES}

(detailed later in this section) involved going over several content analyses that had studied similar concepts to my own and trialling draft versions of the coding scheme. This process was reworked until I was confident that as much relevant information was being extracted from the cover pages as possible. Originally, my coding scheme drew inspiration from a range of research work (Colson-Smith, 2005; Frith, Shaw \& Cheng, 2005; Lindner, 2004; Pawlowski, 2007; Soley \& Kurzbad, 1986). This approach allowed for the scope I was looking for but combining coding categories from so many sources led to an issue in operationalising the features.

To overcome this issue I decided to refine the coding scheme with the intention of preserving the scope without saturating the data with too many coding categories. Locating the study by Hatton and Trautner (2011) aided this process immensely. This study examined cover pages across time with a specific focus on sexualisation. Their work was the closest comparison to the sexualisation part of my study and included many of the features I had identified as important in the trialling phase. Using their work as a starting point for my own, I added in elements that were necessary for my coding scheme and removed features that were shown in testing to be less relevant to the current content.

The codes for examining gender roles were developed in a similar fashion. Most of the literature I engaged with in developing this section of the coding scheme used modified versions of Goffman's (1979) framework (Kang, 1997; Lindner, 2004). Lindner (2004), alongside Goffman's 1979 coding categories, also utilised codes from more recent content analyses that reflect the subtle changes that have occurred in depiction of gender stereotypes in more modern images (Kang, 1997; Lindner, 2004). The similarities between these previous studies led to the decision that the coding scheme from Lindner (2004) could be used without modification for my study as the framework incorporated both old and new features of gender role stereotyping that are found in images of women.

Limitations of the methodological approach are discussed at the end of this chapter. Critiques of the use of content analyses in general and also specific issues that may have arisen because of the coding scheme designed for this study are also detailed there.

\section{Magazine Selection}

The magazines selected for the current study were chosen for their longevity, popularity, and because together they cover a wide range of age demographics. The New Zealand Woman's Weekly (NZWW) is New Zealand's longest running women's magazine. Its current circulation figures are at 52,904 with an estimated readership of 634,000 (Bauer 


\section{LOOK AT HER: WOMEN IN WOMEN'S MAGAZINES}

Media New Zealand, 2016a). The target demographic is women who are in the 40+ age bracket, and the magazine has weekly issues that span the entire time period the current study is focussed on examining (1975-2015).

New Zealand's Woman's Day (NZWD) is currently the top selling New Zealand based women's magazine. The current circulation is at 90,037 with an estimated readership of 633,000 (Bauer Media New Zealand, 2016b). The target demographic is women who are in the 20-49 age bracket. NZWD has been published weekly since 1989 until the present day so there is no available data for the first of the time periods (1975).

Cosmopolitan Australia (Cosmopolitan) was first published in 1972 and is still in circulation at present. This is the only magazine in my sample that has not been specifically published in New Zealand but it has been available in the country since the Australian version of the worldwide magazine was established. The 2015 circulation figures are 64,278 in New Zealand alone, with an estimated readership of 280,000 (Bauer Media Australia, 2016). The target audience of this magazine are women in the 18-35 year old age bracket and it is published monthly.

CLEO magazine was originally a monthly Australian publication that was first printed in 1972. A New Zealand branch of the magazine, CLEO NZ, was established in 1995 and recently closed down with its last issue published in March 2016. The 2015 circulation figures were 6,557 with an estimated readership of 59,000 (Bauer Media New Zealand, 2015). The target audience was women aged between 18 and 29. For this study the cover pages will be analysed throughout the time period as the Australian version was available in New Zealand during the 1970s. Within this thesis the magazine will be referred to as CLEO as during collection and analysis there was no separation of the Australian and New Zealand version.

Girlfriend magazine is the final magazine included in the sample. This magazine is targeted at teenage girls in the 13-19 age bracket. Girlfriend is currently the most popular teen magazine in New Zealand with circulation figures at 19,121 and an estimated readership of 185,000 (Pacific Magazines, 2016). Girlfriend has been published monthly from 1999 until the present day.

Taken together these magazines target the age range of 18-40+ across the study's time frame (1975-2015) and include the bracket of 13-19 years old for the last three year groups (1999, 2005, and 2015). An analysis of a sample of these should therefore provide a good overview of the messages with regards to gender roles and sexualisation that exist in media targeted at women audiences across this time frame. 


\section{Sample Overview}

To achieve a holistic overview of my timeframe, magazines will be analysed from the years $1975,1985,1995,2005$, and 2015 . The exception to this will be where magazines have been published outside of this timeframe (e.g. NZWD was first issued in 1989 so magazines from this year will be included in the 1985 data collection and Girlfriend was first published in 1999 so data from this year will be included in the 1995 bracket).

The magazines within this sample are a mix of weekly and monthly publications. To ensure a similar sample size for each magazine, two approaches to sample selection were used. For monthly publications (Cosmopolitan, CLEO, and Girlfriend), all available covers from the years specified above were analysed. For weekly publications ( $N Z W W$, and $N Z W D)$, months were randomly selected for analysis. Stratified random sampling was used to aid this selection process. Magazines often have seasonal content and therefore having a month from each season was important to get a complete overview of cover pages within each year. The 12 months of the year were split into seasonal strata (spring, summer, autumn, winter) and for each season a month was randomly selected by a computer generator. In New Zealand the spring months are September, October and November; summer covers December, January and February; autumn is March, April, May; and winter is June, July and August (NZ Tourism Guide, 2016). The random selection resulted in magazine covers from each week, of March (autumn), August (winter), November (spring) and December (summer) forming the sample.

The materials (cover pages) for this study were sourced from the archives of Alexander Turnbull Library: National Library of New Zealand (New Zealand based magazines) and the State Library of New South Wales (Australian published magazines). Full colour copies or photographs of the original cover pages were used to allow for annotation and analysis.

Based on the number of issues in each month, for each year the initial sample size was 311 magazine covers. During data collection 43 magazine issues were found to be missing from the archives of the National Library of New Zealand or the State Library of New South Wales. This left a sample size of 270. Table 1 provides a breakdown of the number of issues across year and magazine. Of the total number a further 18 were excluded from the main analyses as the cover photos were unable to be coded (e.g. where the main image was of a solo man). 
Table 1.

Sample Size by Magazine and Year

\begin{tabular}{|c|c|c|c|c|c|c|}
\hline Year & $N Z W W$ & $N Z W D$ & Cosmopolitan & CLEO & Girlfriend & Total \\
\hline 1975 & 15 & N/A & 2 & 10 & N/A & 27 \\
\hline 1985 & 15 & 14 (1989) & 12 & 10 & N/A & 51 \\
\hline 1995 & 15 & 13 & 11 & 12 & 3 (1999) & 54 \\
\hline 2005 & 17 & 17 & 12 & 11 & 10 & 67 \\
\hline 2015 & 18 & 18 & 12 & 12 & 11 & 71 \\
\hline Total & 80 & 62 & 49 & 55 & 24 & 270 \\
\hline
\end{tabular}

\section{Summary of Research Design}

As noted above, the first of the two concepts that are being examined in this study is the sexualisation of women. Sexualisation within the context of this study refers to the framing of women as sexual objects (Calogero et al., 2011). In this study the specific focus is on the extent to which images of women in women's magazines are sexualised. Drawing primarily from the work of Hatton and Trautner (2011), an adapted version of their sexualisation coding scheme was used for this study. In the original article the authors regard sexualisation on cover pages as operating on a continuum; where low scores indicate low sexualisation and higher scores indicate high sexualisation within the image (each category's continuum has its own range). Each category developed had its own spectrum of sexualisation scores that coders' judged and these scores were then added to form an overall score for the cover page as a whole. The sexualisation part of the coding scheme for this study works on the same principle and is detailed later in this chapter.

The second concept to be examined is gender roles. This refers to the beliefs held by society about individuals based solely on their gender. These can be both descriptive, indicating what men and women usually do, and prescriptive, indicating what each gender should do (Eagly, 2009). To explore gender roles, coding categories from Lindner (2004) were utilised. These categories are based on Goffman's (1979) work in analysing advertisements to assess subtle messages about gender relations. These categories were originally scored on a yes-no-NA system with 'yes' indicating the presence of the feature, 'no' indicating the absence of the feature, and NA representing images where a judgment cannot be made. During the course of the analysis this scoring system was adapted to allow for parametric testing (detailed in the gender roles coding scheme summary in a later section of this chapter). 
The Code Book and Coding Checklist have been included in the Appendices section of this thesis; these two resources were developed for data collection and used throughout the coding process.

\section{Coding Schedule}

A coding schedule is a tool of content analysis to develop categories for the data which then allows it to be assessed quantitatively. Using previous studies that have explored similar concepts to what this paper is examining, a coding schedule compromising three main sections was developed.

The two guiding articles that have been heavily drawn on for the methodology of this study are the 2011 analysis of Rolling Stone magazine covers by Hatton and Trautner and the 2004 study by Lindner that analysed gender roles in advertising using aspects from Goffman's 1979 Gender Advertisements work.

\section{Sexualisation}

Hatton and Trautner (2011) conducted a longitudinal study that examined the sexualisation of both men and women on the covers of Rolling Stone magazine from the 1960s until the 2000s. Rolling Stone magazine is an American publication focussed on the full spectrum of popular culture (music, film, television, current affairs). Hatton and Trautner's (2011) original coding scheme had 11 coding categories: clothing/nudity, touch, pose, mouth, breast/chest, genitals, buttocks, text, head vs. body shots, sex acts, and sexual role play. Each of these categories could be scored on a spectrum of sexuality which would be tallied to result in an overall sexualisation score for the cover page.

Prior to the start of the content analysis, sample coding was done to ensure the coding scheme was suitable for the material that would be examined in the current study. Due to the differences in the content of the current sample of magazines, two categories were removed from Hatton and Trautner's coding scheme. The removal of sex acts and sexual role play is based on the lack of presence of these when sample testing was conducted.

During sample testing it was also decided that the facial expression of the model needed to be recognised in the sexualisation score. Facial expression alludes to the proposed relationship between the model and the person viewing the image. It was observed during sample testing that a model's face was a feature point of the cover pages and that there was a difference in perception of sexualisation that was tied to the expression of the model (Bell 2001; Shim, Kwon \& Cheng, 2015). This coding category was operationalised for this study in the same way as the codes derived from Hatton and Trautner (2011). 


\section{LOOK AT HER: WOMEN IN WOMEN'S MAGAZINES}

With these changes made to the original coding scheme, nine coding categories remained (clothing/nudity, touch, pose, mouth, breasts, genitals, buttocks, head vs. body shot, and text) and one new category (facial expression) was added. Each was scored individually for sexualisation, combined, and a cumulative score for the cover page as a whole was generated. These ten coding categories are now each outlined.

\section{Clothing/Nudity}

This category pertains to the level of dress of the person on the cover page (Hatton and Trautner, 2011). This coding category is scored out of five. A score of 0 indicates unrevealing clothing whereas a score of 5 signifies nakedness (or minimal clothing such as just socks/shoes). A score of 1 would be applied where the subject is wearing clothing that is modest, such as low necklines/exposed arms and/or shoulders. A score of 2 would be applied to a moderately revealing image, for example exposed midriffs. A score of 3 indicates highly revealing and/or skin tight clothes. To score a 4 the subject would have to be clothed in apparel that most would not consider clothing (e.g. lingerie, swimwear).

\section{Touch}

Touch, within the coding scheme is a concept that can refer to self-touch, touching others, and being touched (Hatton \& Trautner, 2011). A 4-point scale for sexualisation is used within this category. Scores of 0 indicate no touch of any kind, a score of 3 signifies sexually explicit touching (by self or other) which is defined as the touching/covering of an intimate area. A score of 1 indicates casual touching (e.g. clasping own hands, resting arm on shoulder of another). A score of 2 refers to provocative touching defined as self or other touching that suggests potential sexual contact (e.g. hand resting on thigh of another).

\section{Pose}

Hatton and Trautner (2011) derive this category from the work of Goffman (1979) which suggests that pose is an inherent part of the sexualisation of images. Pose refers to the positioning of the subject and is ranked on a 3-point scale to indicate how suggestive of sexual activity it is. A score of 0 refers to a pose that is in no way related to sexual activity (e.g. standing or engaged in another non-sexual activity). A score of 1 indicates suggested/invited sexual activity (e.g. lifting arms overhead in absence of activity that would require this or any kind of leaning or sitting). A score of 2 indicates an overt positioning of the subject for sexual activity (e.g. lying down, legs spread wide apart). 


\section{Mouth}

The mouth is a facial feature that has been noted to indicate a high level of sexualisation in images and can also indicate 'licensed withdrawal' (described in the Gender Roles section of this chapter), which is a feature of Goffman's gender analysis (Pawlowski, 2007). Based on Hatton and Trautner's (2011) scale, this is coded on a 3-point scale with 0 indicating no suggestion of sexual activity (e.g. closed lips, broad smiles, active use of mouth (e.g. talking or singing)). A score of 1 refers to somewhat sexualised mouth positioning (e.g. slightly parted lips). A score of 2 is indicative of explicitly suggestive mouth positioning (e.g. passive wide open mouths, tongue showing, or something in the mouth).

\section{Breasts/Genitals/Buttocks}

These features have been grouped into one explanation as for each the coding category requires a judgement of whether these regions are the focal point of the image. Each feature is coded on a 3-point scale with 0 indicating that particular body part is not visible and/or not the focal point of the image. A score of 1 refers to an image where the body part is the focal point but is fully covered. A score of 2 indicates the body part is the focal point of the image and is exposed/suggestive of exposure (e.g. unbuttoning of clothes).

\section{Head/Body Shot}

The type of image used is also an important feature to examine. Whether the cover image is a head shot of the model (including images that are also of the subject's head and shoulders only) or an image that shows more of their body can denote the level of sexualisation (Hatton \& Trautner, 2011). Studies have shown that body shots are tied to increased sexualisation because where emphasis on the face can personalise a women in an image, increasing focus on the body can do the opposite (Lambiase \& Reichert, 2006; Schwarz \& Kurz, 1986). Images that personalise women are typically perceived as less sexually objectifying (Lambiase \& Reichert, 2006). As per Hatton and Trautner's (2011) coding scheme, head shots score 0 on the scale and body shots score 1 .

\section{Text}

The text category refers to the text that directly relates to the main image on the cover page and was designed by Hatton and Trautner (2011) to assess the level of sexual innuendo in the caption. Coded on a 3-point scale, scores of 0 refer to the absence of any sort of sexual reference/innuendo in the caption. Scores of 1 indicate the caption contains a sexual innuendo - an allusion to a sexualised topic of some sort (e.g. From Cosmopolitan, September 2015: 
"Lauren Conrad talks style and scandal"). Scores of 2 show that there is an explicit reference to sex or sexuality (e.g. From NZWD, December $18^{\text {th }} 1995$ : "Madonna Uncensored: My KINKY sex romps with LOTS of superstars").

\section{Facial Expression}

The facial expression category refers to the relationship suggested between model and viewer based on the model's gaze. Bell (2001), described four types of relationship suggested by expression. Offer/ideal, where the model offers herself as an idealised version of a certain attribute and their gaze is directed away from the viewer. Demand/affiliation, where the model holds a straight line of direct eye contact with the viewer, smiling or not smiling. Demand/submission, where the model looks down at the viewer, not smiling, and demand/seduction, where the model looks up at the viewer with a tilted head, smiling or pouting. To operationalise in the same way as Hatton and Trautner (2011), these codes had to be ranked for sexualisation scores, and assigning numerical values to these features enabled the overall sexualisation score to be used as a representation of the entire coding scheme.

It was decided that the facial expression with the lowest sexualisation was demand/affiliation. Of the four facial expressions it gives the most personalisation and power to the model, with these two features linked to lower levels of sexualisation as personalisation gives the model purpose beyond being looked at (Bell, 2001; Pawlowski, 2007). Offer/ideal is the next category as the model is given status and power in the image. What they are portraying is an ideal standard of being and whilst this is objectifying, the aloofness of the facial expression reduces the sexualisation (Bell, 2001). Demand/submission was judged to be the second highest category because this facial expression denotes sexual submissiveness, an invitation to the viewer to 'come hither' (Bell, 2001). Demand/seduction suggests agentic sexuality which has been judged as the highest ranking facial expression in regards to sexualisation as the model is actively expressing sexual desire through their facial expression (Bell, 2001).

In line with the scoring system from Hatton and Trautner (2011), demand affiliation is scored as 0 , offer/ideal is scored as 1 , demand/submission is scored as 2 , and demand/seduction is scored as 3 .

\section{Gender Roles}

Lindner (2004) performed a longitudinal content analysis examining the portrayal of women in advertisements and comparing this between general interest and women's fashion magazines over a 50 year period (1955 - 2002). A coding scheme was developed that drew 


\section{LOOK AT HER: WOMEN IN WOMEN'S MAGAZINES}

heavily from the work of Goffman (1979) which focusses on examining non-overt messages about gender roles. Goffman's original work specifies five categories designed to capture these subtle communications: relative size, function ranking, feminine touch, ritualisation of subordination, and licensed withdrawal. Alongside these Lindner (2004) adds four categories: body display (originally added to the analysis by Kang, 1997), movement, location (originally added by Umiker-Sebeok, 1996), and objectification, which was created specifically by Lindner (2004). For this study the coding scheme from Lindner (2004) was replicated. All codes are scored on a yes-no-NA (not applicable) basis - NA is utilised where categories require both a man and woman to be present in the image and this dynamic does not occur. As mentioned earlier, this categorical scale was adapted to an additive one during the course of the analysis to allow for parametric testing. Creating a system that allowed for a total score to be analysed means that within this analysis the theme of 'gender roles' can be looked at as a holistic variable as well as individual categories. The total score was calculated by allocating one 'point' for a "yes" response on the original scale. If a feature was coded as "no" or "NA" a score of zero was given. Frequency data is reported with the original scoring system so differences between "no" and "NA" are not overlooked.

\section{Relative Size}

Relative size can be assessed in images where both genders are present. To code it as present, the man in the image must take up more space than the woman. This can be interpreted as a subtle message of male superiority (Lindner, 2004).

\section{Function Ranking}

Lindner (2004) details that function ranking can again only be assessed in images featuring both men and women. To be coded as present, the man must be shown as the one in control - so in a more powerful executive role in the image or having some form of control over the woman.

\section{Feminine Touch}

Feminine touch can be assessed in any image where touch (of self, object, or other person) is present. It refers to the manner of positioning women to have unnatural touch with their own bodies or objects. The touch itself is unusual in that it is detached from how said object would be utilised in a real-life scenario - often the touch is gentle and caressing rather than controlling/manipulating an object. This is a visual form of objectification because it suggests that the woman in the image is there to show the object rather than actively use it 
(Lindner, 2004). If the touch fits this description, it is to be coded as present but not if there is touch within the image but it involves normal/controlled use of the self/object/other person. If there is no touch within the image it is coded as "NA"

\section{Ritualization of Subordination}

This can be coded in both images of women alone or with a male counterpart. To be coded as present the woman must demonstrate some sort of physical lowering/shrinking of the body (e.g. lying down, canting postures, or sitting). This can also be coded if a male is present and is embracing the female (in a way that restricts movement) or she is leaning on him or using him for support in some way (Lindner, 2004).

\section{Licensed Withdrawal}

This can be coded in any image of a woman. To be judged as present the woman must appear to psychologically remove herself from the situation/context of the image. This can be demonstrated in many ways such as an expansive smile/laughter, covering of the face/mouth, withdrawal of gaze, or engaging in another activity outside of the larger context, such as talking on the phone (Lindner, 2004).

\section{Body Display}

Body display is determined as present by Lindner (2004) in an image where a women is in revealing/minimal/no clothing.

\section{Movement}

Lack of movement can be determined in any image. This is coded as present where a woman is inhibited in her movement (e.g. wrapped in a blanket). Lack of movement is seen as a representation of a lack of control over the occupied space (Lindner, 2004).

\section{Location}

Location is another category that can be coded for in any image. To be coded as present in an image, the woman in the image must either be shown in a domestic location (e.g. kitchen, bedroom, or bathroom) or she is in a decontextualized setting that cannot be identified for any specific/purposeful activities (Lindner, 2004).

\section{Objectification}

Lindner (2004) defines objectification as where the purpose of the woman in the image is to be looked at. This is to be coded as present in images where the woman's major function is to be looked at rather than engaging in any focussed activity. 


\section{Demographic Information}

A variety of demographic information were also collected. These categories include: the gender(s) of those in the main image; age of those in the main image (18-25, 26-35, 3650, 50+); ethnicity (New Zealand European/Caucasian, Maori, Pacific Islander, African American, Asian, Indian, Other (specify)); local/international figure (New Zealand/Australian or from elsewhere); public figure/cover model; whether the image is a staged shot (posed) or a paparazzi/candid image where the subject is not explicitly posing for the photo. Captions were also coded for theme/topic. These themes included: beauty/body image, career, celebrity infatuation, celebrity news, family, fashion, health, home, love/relationship, marriage, money, news, sex, leisure). A more detailed overview of the demographic information collected and how decisions were made can be found in the Code Book (Appendix 1).

\section{Reliability Coding}

Photocopies of the 270 magazine covers were coded by the researcher with another research assistant coding 30 of the overall sample. The research assistant was given an early draft of the methodology chapter of this thesis prior to training with the researcher and the Code Book (Appendix 1) was discussed in detail over a three hour training session prior to the sample being coded. The research assistant was then given a sample of 10 cover pages for training, once coded they were compared to the researcher's and any disagreements were resolved with discussion. The final sample of 30 cover pages were selected through a process of random sampling. One of the five magazines was randomly selected (using a computer generator) for each of the five year groups. Following this the cover pages were then sorted into chronological order, numbered, and the first 30 covers as indicated by a randomised numerical list were used as the reliability sample. Within this sample two covers were excluded as they featured images of men on the cover - the remaining sample of 28 cover pages (approximately $10 \%$ of the total sample) were analysed to establish inter-rater reliability.

Reliability was calculated by looking at agreements and disagreements between the coders within each coding category. Across the covers the average agreement rate for the sexualisation section of the coding scheme was $88.2 \%$. Cohen's Kappa ${ }^{8}$ scores for each individual variable ranged from fair agreement $\kappa=.40, p=.03$ ("touch") to substantial agreement $\kappa=.79, p<.001$ (“facial expression") (Viera \& Garrett, 2005). For some variables

\footnotetext{
${ }^{8}$ Cohen's kappa is a statistical measure of agreement between judgements made by 'raters' who have interpreted the same data (Viera and Garrett, 2005).
} 
one or both raters were 'constant', meaning that across the sample of 28 magazines for that particular variable they had given the same score in that category for each cover. This created an error with the Kappa formula that would not allow for it to be calculated. For these variables ("genitals", "buttocks", and "text") the percentage agreement was used as an indication of reliability. Taking into account Kappa statistics and levels of percentage agreement (see Table 2), all coding categories had an adequate level of reliability within this section of my coding scheme.

Average agreement for the gender roles section of the coding scheme was $91.7 \%$. Cohen's Kappa scores for each individual variable ranged from substantial agreement $\kappa=$ $.63, p<.001$ ("licensed withdrawal") to perfect agreement $\kappa=1.00, p<.001$ ("function ranking”) (Viera \& Garrett, 2005). The 'constant' error caused issues with the Kappa analysis for the "movement" and "objectification" variables in this section of the coding scheme. Also, a non-significant Kappa result was calculated for the variable "location". In these instances the percentage agreements were used as an indication of reliability for these codes. Table 2 displays percentage agreements and Cohen's Kappa results for each coding category. The figures indicate an adequate level of reliability for each code within this part of the coding scheme.

Table 2.

Percentage Agreement and Cohen's Kappa Results by Coding Category

\begin{tabular}{rccc}
\hline \multicolumn{1}{l}{ Coding Category } & Percentage Agreement & Cohen's Kappa \\
Sexualisation & $78.6 \%$ & $\kappa=0.68^{* * *}$ \\
Clothing/Nudity & $85.7 \%$ & $\kappa=0.74^{* * *}$ \\
Touch & $71.4 \%$ & $\kappa=0.40^{*}$ \\
Pose & $85.7 \%$ & $\kappa=0.58^{* *}$ \\
Mouth & $85.7 \%$ & & $\kappa=0.79^{* * *}$ \\
Facial Expression & $96.4 \%$ & & $\kappa=0.74^{* * *}$ \\
Breasts & $100 \%$ & rater constant error \\
Genitals & $100 \%$ & rater constant error \\
Buttocks & $82.1 \%$ & & $\kappa=.62^{* *}$ \\
Tead/Body Shot & $96.4 \%$ & rater constant error \\
\hline
\end{tabular}




\begin{tabular}{rcc} 
Gender Roles & & \\
Relative Size & $89.3 \%$ & $\kappa=0.706^{* * *}$ \\
Function Ranking & $100 \%$ & $\kappa=1.00^{* * *}$ \\
Feminine Touch & $89.3 \%$ & $\kappa=0.727^{* * *}$ \\
Ritualisation of & $89.3 \%$ & $\kappa=0.779 * * *$ \\
Subordination & $92.9 \%$ & $\kappa=0.632^{* * *}$ \\
Licensed Withdrawal & $96.4 \%$ & $\kappa=0.837^{* * *}$ \\
Body Display & $78.6 \%$ & rater constant error \\
Movement & $92.9 \%$ & $\kappa=-.037, n s$ \\
Location & $96.4 \%$ & rater constant error \\
\hline Objectification & &
\end{tabular}

Note. $* p<.05, * * p<.01, * * * p<.001$. Percentages rounded to $1 \mathrm{dp}$.

\section{Limitations}

Content analysis, whilst the most appropriate way to examine data like that presented in this thesis, has some inherent flaws. Judgement on categories is to some extent always influenced by personal experience, knowledge and values (Macnamara, 2005). Where the primary researcher and coder are one and the same this can potentially result in a certain level of bias (Kolbe \& Burnett, 1991). The use of reliability coding heightens the validity of these results as it indicates the ability for the data to be replicated which implies little subjectivity and/or bias within the dataset. However, as the secondary coder was trained by the primary researcher there is the potential that the secondary coder was trying to provide results that the researcher wanted instead of providing a truly objective judgement of the data. One step that researchers can take to overcome the hurdle of subjectivity and researcher bias is to employ blind coders for data collection and reliability analysis. These coders would rely solely on a code book to make judgements on the data and would have no knowledge of the research objectives whilst the coding process was being undertaken (Kolbe \& Burnett, 1991). Due to both timing and funding this method was outside the bounds of the current research.

The second limitation of content analysis in general is that the act of quantifying data that is qualitative in nature will almost always result in a loss of detail and depth (Krippendorff, 1989). The use of directed content analysis in this research (where coding categories are established prior to data collection) may have led to a loss of unique data within this sample (Krippendorff, 1989; Macnamara, 2005). Whilst analysing images by specific features increases reliability and replicability of data, the holistic examination of 
LOOK AT HER: WOMEN IN WOMEN'S MAGAZINES

cover pages may have resulted in a descriptively richer (albeit significantly more subjective) dataset.

The next chapter details the analyses undertaken, the descriptive and inferential statistics produced, and some qualitative examples of cover pages from the sample to help highlight the significant differences found. 


\section{Chapter Four: Results}

The first part of this section provides an overview of the initial checks that were conducted on the dataset. These give an indication of what the data look like and the rationale behind the choice of analyses. The second section provides an overview of frequency data and average scores for sexualisation and gender role scores both in total and across year groups. The third section presents the results generated from a series of statistical tests on total sexualisation and gender role scores. Example cover pages are used throughout this chapter to illustrate key differences within the sample.

Both key themes (sexualisation and gender roles) were looked at in respect to their individual features (with descriptive statistics) and as holistic concepts (with parametric analyses). In order to examine patterns in the depiction of women in cover page images, scores were compared across year groups, magazines, and other demographic variables collected within the coding scheme.

\section{Preliminary Analyses}

Normality tests were conducted on the overall sexualisation score and gender role score variables as well as on these variables when the dataset was split into year groups. Table 3 shows the results of the Kolmogorov-Smirnov test which assesses the distribution of the data and Levene's tests which calculate the equality of variance. The results indicate that whilst equal variances can be assumed for both sexualisation and gender roles scores, most variables do not meet the assumption for data distribution which indicates that the data are not normally distributed. Due to the nature of the dataset, removing outliers would not have been appropriate and transforming the data tended to improve the Levene's statistic rather than correcting the normality of the distribution. Histogram plots show a positive skew within the data, however, there was a reasonable range of variability so robust parametric tests were used on the raw data (Field, 2009). 
LOOK AT HER: WOMEN IN WOMEN'S MAGAZINES

Table 3.

Normality Tests

\begin{tabular}{|c|c|c|}
\hline Variable & Kolmogorov-Smirnov Statistic & Levene's Statistic \\
\hline Overall Sexualisation & $D(252)=.14, p<.001^{*}$ & \\
\hline Sexualisation -1975 & $D(22)=.21, p=.01^{*}$ & \\
\hline Sexualisation - 1985 & $D(44)=.13, p=.06$ & \\
\hline Sexualisation - 1995 & $D(53)=.13, p=.02^{*}$ & $F(4,247)=2.09, p=.08$ \\
\hline Sexualisation -2005 & $D(67)=.11, p=.05$ & \\
\hline Sexualisation - 2015 & $D(66)=.18, p<.001^{*}$ & \\
\hline Overall Gender Roles & $D(252)=.21, p<.001^{*}$ & \\
\hline Gender Roles - 1975 & $D(22)=.18, p=.06$ & \\
\hline Gender Roles - 1985 & $D(44)=.26, p<.001 *$ & \\
\hline Gender Roles - 1995 & $D(53)=.23, p<.001 *$ & $F(4,247)=1.46, p=.21$ \\
\hline Gender Roles - 2005 & $D(67)=.20, p<.001^{*}$ & \\
\hline Gender Roles - 2015 & $D(66)=.20, p<.001 *$ & \\
\hline
\end{tabular}

Note. ${ }^{*}=p<.05$, result does not meet normality assumptions.

\section{Descriptive Statistics}

Frequency data for the demographic variables of the cover image as well as the theme of the image caption are reported in Table 4. This data shows that across all time periods the typical woman depicted on cover pages is a solo, New Zealand European woman, aged between 18 and 35. This pattern will be discussed in more detail in the following chapter. Another interesting feature was the image caption frequency, which shows the overwhelming emphasis of celebrity culture within the images used on the cover of women's magazines. 
LOOK AT HER: WOMEN IN WOMEN'S MAGAZINES

Table 4.

Frequency Data for Demographic Variables across Year Groups

\begin{tabular}{|c|c|c|c|c|c|c|c|c|c|c|c|c|}
\hline \multirow[b]{2}{*}{ Variable } & \multicolumn{2}{|c|}{1975} & \multicolumn{2}{|c|}{1985} & \multicolumn{2}{|c|}{1995} & \multicolumn{2}{|c|}{2005} & \multicolumn{2}{|c|}{2015} & \multicolumn{2}{|c|}{ Total } \\
\hline & $\mathrm{N}$ & $(\%)$ & $\mathrm{N}$ & $(\%)$ & $\mathrm{N}$ & $(\%)$ & $\mathrm{N}$ & $(\%)$ & $\mathrm{N}$ & $(\%)$ & $\overline{\mathbf{N}}$ & $(\%)$ \\
\hline \multicolumn{13}{|l|}{ Gender(s) } \\
\hline Solo Female & 14 & (51.9) & 38 & $(74.5)$ & 44 & $(81.5)$ & 52 & (77.6) & 54 & (76.1) & 202 & (74.8) \\
\hline Solo Male & 5 & (18.5) & 7 & (13.7) & 1 & (1.9) & 0 & $(0.0)$ & 4 & (5.6) & 17 & (6.3) \\
\hline Group Mixed & 8 & (29.6) & 5 & $(9.8)$ & 7 & (13.0) & 12 & (17.9) & 8 & (11.3) & 40 & (14.8) \\
\hline Group All Male & 0 & $(0.0)$ & 0 & $(0.0)$ & 0 & $(0.0)$ & 0 & $(0.0)$ & 1 & (1.4) & 1 & $(0.4)$ \\
\hline Group All & 0 & $(0.0)$ & 1 & (2.0) & 2 & (3.7) & 3 & $(4.5)$ & 4 & (5.6) & 10 & (3.7) \\
\hline
\end{tabular}

Age

$\begin{array}{rcccccccccccc}\text { Under 18 } & 0 & (0.0) & 0 & (0.0) & 0 & (0.0) & 1 & (1.5) & 0 & (0.0) & \mathbf{1} & (\mathbf{0 . 4}) \\ 18-25 & 3 & (11.1) & 21 & (41.2) & 24 & (44.4) & 25 & (37.3) & 18 & (25.4) & \mathbf{9 1} & \mathbf{( 3 3 . 7 )} \\ 26-35 & 13 & (48.1) & 18 & (35.3) & 22 & (40.7) & 24 & (35.8) & 29 & (40.8) & \mathbf{1 0 6} & \mathbf{( 3 9 . 3}) \\ 36-50 & 3 & (11.1) & 4 & (7.8) & 4 & (7.4) & 15 & (22.4) & 10 & (14.1) & \mathbf{3 6} & \mathbf{( 1 3 . 3}) \\ 50+ & 3 & (11.1) & 1 & (2.0) & 3 & (5.6) & 2 & (3.0) & 9 & (12.7) & \mathbf{1 8} & \mathbf{( 6 . 7 )}\end{array}$

Ethnicity

$\begin{array}{rcccccccccccc}\text { NZ European } & 20 & (74.1) & 41 & (80.4) & 50 & (92.6) & 60 & (89.6) & 54 & (76.1) & \mathbf{2 2 5} & \mathbf{( 8 3 . 3 )} \\ \text { Maori } & 1 & (3.7) & 1 & (2.0) & 1 & (1.9) & 0 & (0.0) & 3 & (4.2) & \mathbf{6} & \mathbf{( 2 . 2 )} \\ \text { Pacific Islander } & 0 & (0.0) & 0 & (0.0) & 1 & (1.9) & 1 & (1.5) & 2 & (2.8) & \mathbf{4} & \mathbf{( 1 . 5 )} \\ \text { African } & 0 & (0.0) & 1 & (2.0) & 0 & (0.0) & 1 & (1.5) & 1 & (1.4) & \mathbf{3} & \mathbf{( 1 . 1 )}\end{array}$

American

$\begin{array}{lllllllllllll}\text { Asian } & 1 & (3.7) & 1 & (2.0) & 1 & (1.9) & 0 & (0.0) & 2 & (2.8) & \mathbf{5} & \mathbf{( 1 . 9 )} \\ \text { Other } & 0 & (0.0) & 0 & (0.0) & 0 & (0.0) & 5 & (7.5) & 4 & (5.6) & \mathbf{9} & \mathbf{( 3 . 3}\end{array}$

Image Caption

$\begin{array}{lllllllllllll}\text { Celeb Infatuation } & 5 & (18.5) & 11 & (21.6) & 19 & (35.2) & 57 & (85.1) & 25 & \text { (35.2) } & 117 & \mathbf{( 4 3 . 3 )}\end{array}$

$\begin{array}{lllllllllllll}\text { Celeb News } & 7 & (25.9) & 14 & (27.5) & 13 & (24.1) & 10 & (14.9) & 43 & (60.6) & 87 & \mathbf{( 3 2 . 2 )}\end{array}$

$\begin{array}{lllllllllllll}\text { Fashion } & 0 & (0.0) & 0 & (0.0) & 1 & (1.9) & 0 & (0.0) & 0 & (0.0) & 1 & (0.4)\end{array}$

$\begin{array}{lllllllllllll}\text { Love/relationship } & 0 & (0.0) & 1 & (2.0) & 0 & (0.0) & 0 & (0.0) & 0 & (0.0) & 1 & (\mathbf{0 . 4})\end{array}$

$\begin{array}{lllllllllllll}\operatorname{Sex} & 0 & (0.0) & 0 & (0.0) & 1 & (1.9) & 0 & (0.0) & 0 & (0.0) & 4 & (1.5)\end{array}$

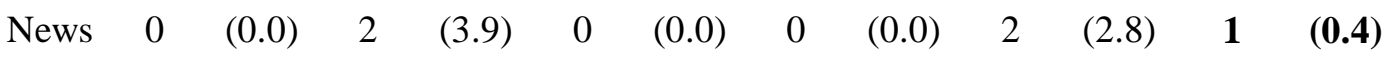

$\begin{array}{lllllllllllll}\text { Leisure } & 4 & (14.8) & 0 & (0.0) & 0 & (0.0) & 0 & (0.0) & 0 & (0.0) & 4 & (\mathbf{1 . 5})\end{array}$

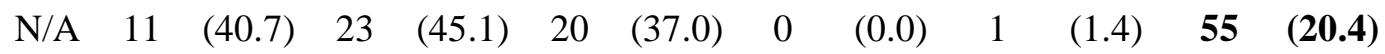

Note. Male covers were excluded from the age and ethnicity data collection so the total $\mathrm{N}$ for these data is 252 rather than 270 . 
Frequency data for each individual code within the sexualisation and gender roles coding schemes are displayed in Tables 5 and 6 . Whilst the parametric analyses (detailed in the next section) were computed on the total scores for each section of the coding scheme, frequency data for each code is presented to give a more detailed picture of the data.

Frequency data show a clustering of data on the low scoring ends of each individual code within the sexualisation section of the coding scheme. One interesting feature of note is within the "facial expression" code where the most frequent score is for "demand/affiliation" (57.8\%) but the second most frequent score is "demand/seduction" (20.7\%) (See Figure 1 of examples). This suggests that, for facial expression, images of women are likely to be either personal or highly sexualised with very few images sitting in the middle of this scale.

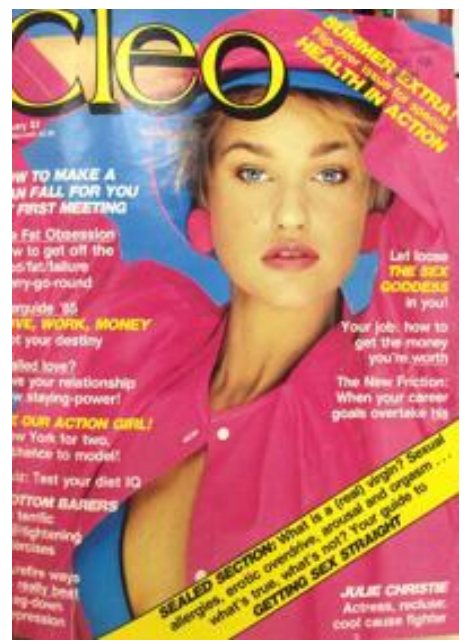

Demand/affiliation, score $=0$

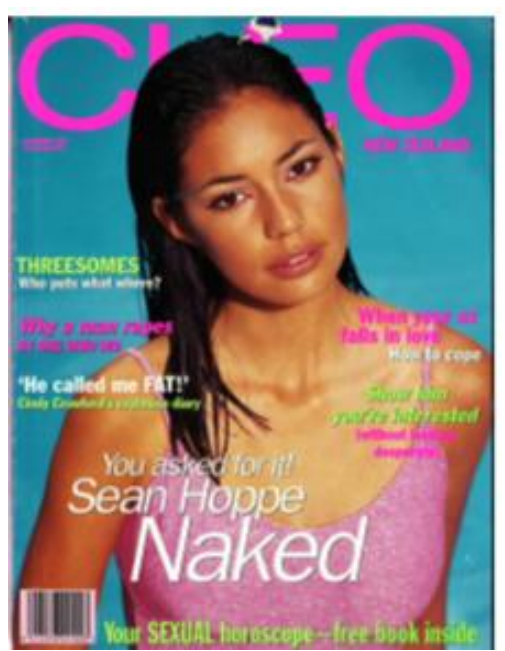

Demand/seduction, score $=3$

Figure 1. Examples of the two most common facial expression codes.

Left: From CLEO January 1985. Copyright 1985 by Bauer Media Pty Limited. Reprinted with permission. Right: From CLEO November 1995. Copyright 1995 by Bauer Media Pty Limited. Reprinted with permission.

I have highlighted this result because the absence of a middle ground between nonsexualised and overtly sexualised facial expressions could be a manifestation of the polarisation between "good" and "bad" women. This polarisation is known as the 'Madonna/whore' dichotomy and exists largely due to traditional gendered prescriptions around sex and sexual desire (Tolman, 1994). The dichotomy refers to the idea that women are either 'good' (Madonna) because they adhere to the traditional feminine role of controlling sexual desires or 'bad' (whore) because they actively pursue their sexual urges (Tolman, 1994). To see these two extremes represented in the facial coding category is concerning given that women's magazines have a role in creating the image of the 'ideal' 


\section{LOOK AT HER: WOMEN IN WOMEN'S MAGAZINES}

woman and reinforce ideas about what it means to be a woman (Burton, 2005; Croteau \& Hoynes, 2014). However, the 'facial expression' code is just one part of the overall sexualisation measure. The implications of the sexualisation of women on women's expressions of their own sexuality are discussed holistically in more detail in the next chapter. 
LOOK AT HER: WOMEN IN WOMEN'S MAGAZINES

Table 5.

Frequency Data for Each Sexualisation Code across Year Groups

\begin{tabular}{|c|c|c|c|c|c|c|c|c|c|c|c|c|}
\hline \multirow{2}{*}{ Code } & \multicolumn{2}{|c|}{1975} & \multicolumn{2}{|c|}{1985} & \multicolumn{2}{|c|}{1995} & \multicolumn{2}{|c|}{2005} & \multicolumn{2}{|c|}{2015} & \multicolumn{2}{|r|}{ Total } \\
\hline & $\overline{\mathrm{N}}$ & $(\%)$ & $\overline{\mathrm{N}}$ & $(\%)$ & $\overline{\mathrm{N}}$ & $(\%)$ & $\overline{\mathrm{N}}$ & $(\%)$ & $\overline{\mathrm{N}}$ & $(\%)$ & $\mathbf{N}$ & $(\%)$ \\
\hline \multicolumn{13}{|l|}{ Clothing/Nudity } \\
\hline Unrevealing & 11 & $(40.7)$ & 19 & $(37.3)$ & 14 & $(25.9)$ & 10 & $(14.9)$ & 23 & $(32.4)$ & 77 & (28.5) \\
\hline Modest & 3 & (11.1) & 12 & $(23.5)$ & 24 & $(44.4)$ & 24 & $(35.8)$ & 24 & $(33.8)$ & 87 & (32.2) \\
\hline Moderate & 1 & (3.7) & 2 & (3.9) & 4 & (7.4) & 18 & (26.9) & 8 & $(11.3)$ & 33 & $(12.2)$ \\
\hline Highly Revealing & 5 & $(18.5)$ & 7 & $(13.7)$ & 5 & (9.3) & 12 & $(17.9)$ & 8 & (11.3) & 37 & (13.7) \\
\hline Non-clothing & 1 & (3.7) & 4 & (7.8) & 3 & (5.6) & 2 & (3.0) & 3 & $(4.2)$ & 13 & (4.8) \\
\hline Naked & 1 & (3.7) & 0 & $(0.0)$ & 3 & (5.6) & 1 & $(1.5)$ & 0 & $(0.0)$ & 5 & (1.9) \\
\hline \multicolumn{13}{|l|}{ Touch } \\
\hline No Touch & 9 & $(33.3)$ & 21 & $(41.2)$ & 27 & $(50.0)$ & 25 & $(37.3)$ & 15 & $(21.1)$ & 97 & (35.9) \\
\hline Casual & 11 & $(40.7)$ & 19 & $(37.3)$ & 17 & $(31.5)$ & 24 & $(35.8)$ & 39 & $(54.9)$ & 110 & (40.7) \\
\hline Provocative & 2 & (7.4) & 4 & (7.8) & 8 & $(14.8)$ & 17 & $(25.4)$ & 12 & $(16.9)$ & 43 & (15.9) \\
\hline Explicit & 0 & $(0.0)$ & 0 & $(0.0)$ & 1 & (1.9) & 1 & (1.5) & 0 & $(0.0)$ & 2 & $(0.7)$ \\
\hline \multicolumn{13}{|l|}{ Pose } \\
\hline Innocuous & 15 & $(55.6)$ & 25 & $(49.0)$ & 26 & $(48.1)$ & 36 & $(53.7)$ & 37 & $(52.1)$ & 139 & (51.5) \\
\hline Suggestive & 6 & $(22.2)$ & 18 & $(35.3)$ & 22 & $(40.7)$ & 26 & $(38.8)$ & 26 & $(36.6)$ & 98 & (36.3) \\
\hline Explicit & 0 & $(0.0)$ & 1 & $(2.0)$ & 5 & (9.3) & 5 & $(7.5)$ & 3 & $(4.2)$ & 14 & $(5.2)$ \\
\hline
\end{tabular}


LOOK AT HER: WOMEN IN WOMEN'S MAGAZINES

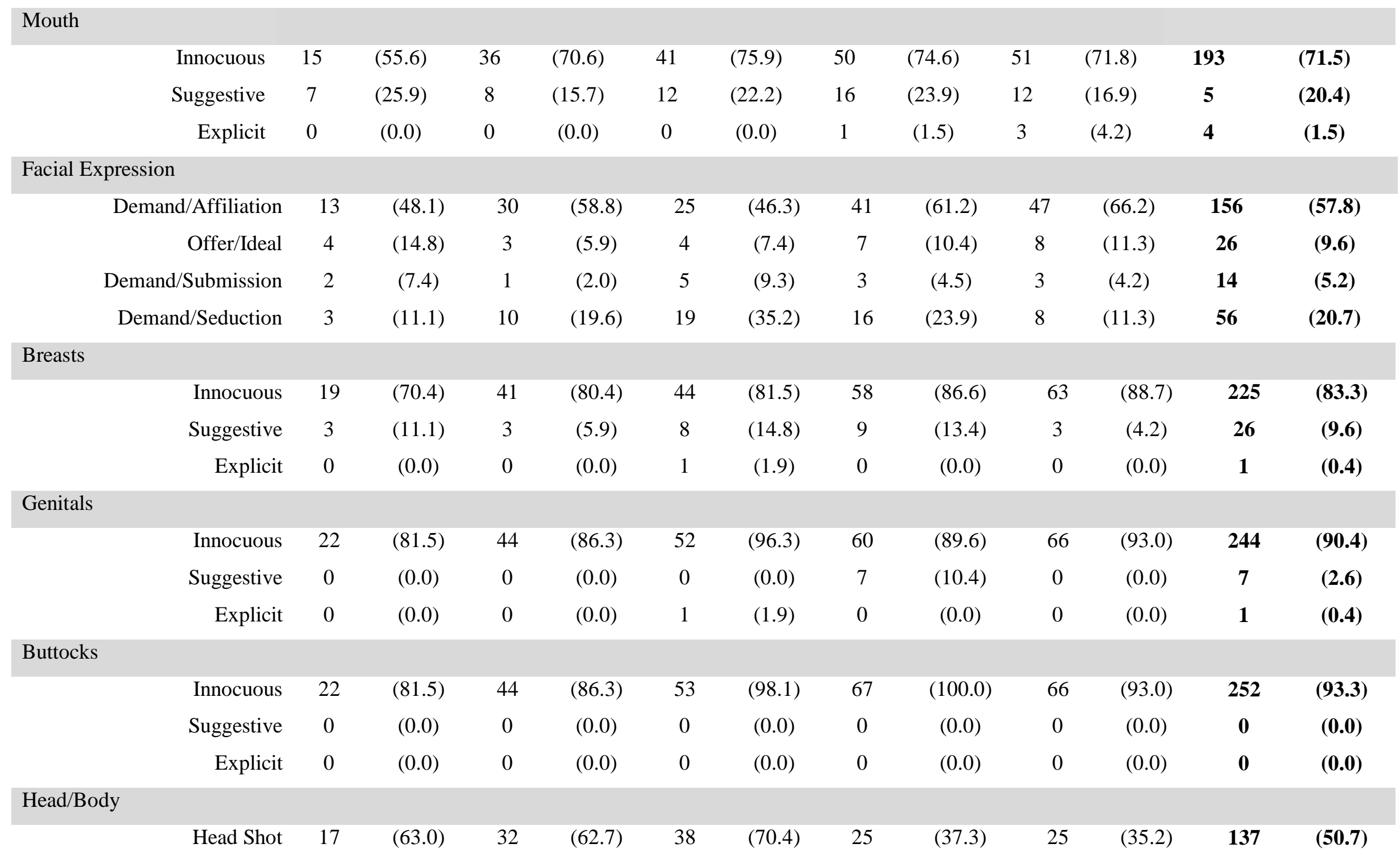


LOOK AT HER: WOMEN IN WOMEN'S MAGAZINES

\begin{tabular}{lcccccccccccccc} 
& Body Shot & 5 & $(18.5)$ & 12 & $(23.5)$ & 15 & $(27.8)$ & 42 & $(62.7)$ & 41 & $(57.7)$ & $\mathbf{1 1 5}$ & $\mathbf{( 4 2 . 6 )}$ \\
Text & & & & & & & & & & \\
& Innocuous & 21 & $(77.8)$ & 38 & $(74.5)$ & 45 & $(83.3)$ & 51 & $(76.1)$ & 56 & $(78.9)$ & $\mathbf{2 1 1}$ & $\mathbf{( 7 8 . 1 )}$ \\
& Suggestive & 1 & $(3.7)$ & 3 & $(5.9)$ & 5 & $(9.3)$ & 13 & $(19.4)$ & 7 & $(9.9)$ & $\mathbf{2 9}$ & $(\mathbf{1 0 . 7})$ \\
& Explicit & 0 & $(0.0)$ & 3 & $(5.9)$ & 3 & $(5.6)$ & 3 & $(4.5)$ & 3 & $(4.2)$ & $\mathbf{1 2}$ & $\mathbf{( 4 . 4 )}$ \\
\hline
\end{tabular}


LOOK AT HER: WOMEN IN WOMEN'S MAGAZINES

Table 6.

Frequency Data for Each Gender Role Code across Year Groups

\begin{tabular}{|c|c|c|c|c|c|c|c|c|c|c|c|c|c|}
\hline \multirow{2}{*}{ Code } & & \multicolumn{2}{|c|}{1975} & \multicolumn{2}{|c|}{1985} & \multicolumn{2}{|c|}{1995} & \multicolumn{2}{|c|}{2005} & \multicolumn{2}{|c|}{2015} & \multicolumn{2}{|c|}{ Total } \\
\hline & & $\mathrm{N}$ & $\overline{(\%)}$ & $\mathrm{N}$ & $(\%)$ & $\bar{N}$ & $\overline{(\%)}$ & $\mathrm{N}$ & $\overline{(\%)}$ & $\bar{N}$ & $\overline{(\%)}$ & $\overline{\mathbf{N}}$ & $(\%)$ \\
\hline \multicolumn{14}{|c|}{ Relative Size } \\
\hline & Yes & 5 & $(18.5)$ & 3 & (5.9) & 4 & (7.4) & 8 & (11.9) & 4 & (5.6) & 24 & (8.9) \\
\hline & No & 3 & (11.1) & 2 & (3.9) & 3 & (5.6) & 4 & $(6.0)$ & 5 & $(7.0)$ & 17 & (6.3) \\
\hline & N/A & 14 & (51.9) & 39 & $(76.5)$ & 46 & $(85.2)$ & 55 & $(82.1)$ & 57 & (80.3) & 211 & (78.1) \\
\hline \multicolumn{14}{|c|}{ Function Ranking } \\
\hline & Yes & 8 & (29.6) & 1 & $(2.0)$ & 2 & (3.7) & 9 & (13.4) & 4 & (5.6) & 24 & (8.9) \\
\hline & No & 0 & $(0.0)$ & 4 & (7.8) & 5 & (9.3) & 3 & $(4.5)$ & 5 & $(7.0)$ & 17 & (6.3) \\
\hline & N/A & 14 & (51.9) & 39 & $(76.5)$ & 46 & $(85.2)$ & 55 & $(82.1)$ & 57 & (80.3) & 211 & (78.1) \\
\hline \multicolumn{14}{|c|}{ Feminine Touch } \\
\hline & Yes & 6 & $(22.2)$ & 15 & $(21.6)$ & 16 & $(29.6)$ & 34 & $(50.7)$ & 34 & $(47.9)$ & 105 & (38.9) \\
\hline & No & 6 & $(22.2)$ & 11 & $(29.4)$ & 13 & $(24.1)$ & 7 & (10.4) & 22 & (31.0) & 61 & (22.6) \\
\hline & N/A & 10 & $(37.0)$ & 18 & (35.3) & 24 & (44.4) & 26 & $(38.8)$ & 10 & (14.1) & 86 & (31.9) \\
\hline \multirow{2}{*}{\multicolumn{14}{|c|}{$\begin{array}{l}\text { Ritualisation of } \\
\text { Subordination }\end{array}$}} \\
\hline & & & & & & & & & & & & & \\
\hline & Yes & 8 & (29.6) & 20 & (39.2) & 32 & (59.3) & 21 & $(31.3)$ & 35 & (49.3) & 116 & (43.0) \\
\hline & No & 14 & (51.9) & 24 & (47.1) & 21 & (38.9) & 46 & (68.7) & 31 & (43.7) & 136 & (50.4) \\
\hline
\end{tabular}


LOOK AT HER: WOMEN IN WOMEN'S MAGAZINES

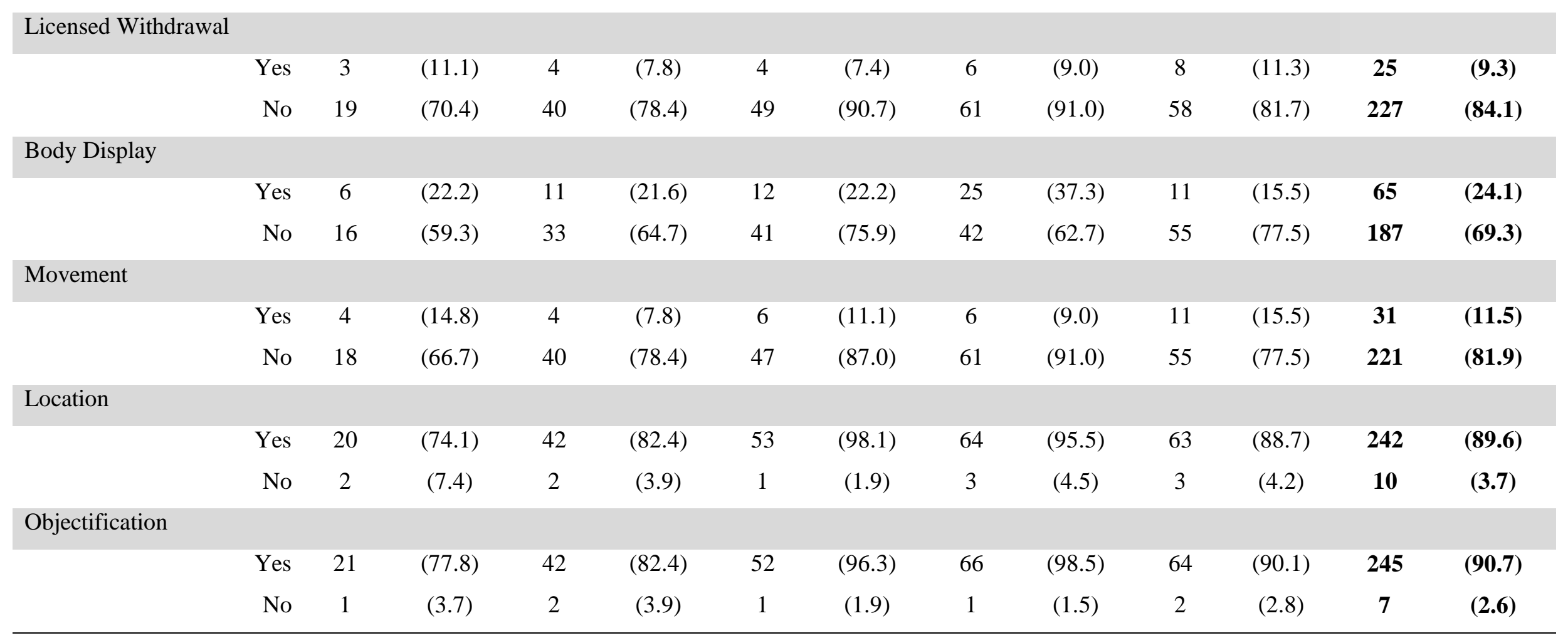

Note. Percentages may not add up to $100 \%$, not reported are covers that were not coded as they depicted a solo man. 
Frequency data for gender role categories also provide some interesting detail. Whilst most codes show a fairly even split within the data, "feminine touch", "location"10, and "objectification" 11 are all frequently used within the images of women in the sample. All three of these codes share a trait: they all relate to context and functionality being removed from images of women. This is consistent with the fifth prediction made for these results, it was expected that the data would show a tendency for stereotypes to be conveyed through the removal of agency from women in images. By 'removal of agency' I am referring to an erasure of purpose from images of women. Removing functional touch, an identifiable location, and focussed activity from images of women eradicates the idea that women have functionality, resigning them instead to decorative, ornamental roles.

Means and standard deviations for sexualisation and gender role scores in total and across the five year groups are shown in Table 7. The scale for sexualisation ranged from 0 to 24, within the current sample the lowest recorded score was 0 and the highest score was 16. Mean scores in the table below indicate that sexualisation scores are low when compared to both the highest and maximum score indicate. However, the high standard deviation speaks to the variability of the data. Figures 1 and 2 (located in the inferential statistics section of this chapter) show example cover pages that scored the average level of sexualisation between time periods and magazines. The results indicate that a low level of sexualisation is present in images on the cover pages of women's magazines. However, Figures 1 and 2 help to show the differences in how sexualisation scores manifest and illustrate the differences between these average scores. Higher numerical scores were expected; however, the presence of even a low level of sexualisation has important implications and supports the first hypothesis of the research.

On the gender roles scale the range was from 0 to 9 and within the current dataset the lowest score was 1 and the highest was 8. Gender role scores on average are also relatively low although some are close to the mid-point of the scale. This is consistent with the third hypothesis of this research.

\footnotetext{
${ }^{9}$ Feminine touch refers to unnatural touch of the subject's own body, another person, or an object. The touch itself is unusual in that it is detached from how said object would be utilised in a real-life scenario.

${ }^{10}$ Location refers to images set in a domestic location (e.g. kitchen, bedroom, or bathroom) or in a decontextualized setting that can't be identified for any specific/purposeful activities.

${ }^{11}$ Objectification refers to images where the sole purpose of the woman is to be looked at (so an absence of any engaged or suggested activity).
} 
LOOK AT HER: WOMEN IN WOMEN'S MAGAZINES

Table 7.

Descriptive Statistics for Sexualisation and Gender Role Scores across Magazines and Year Groups

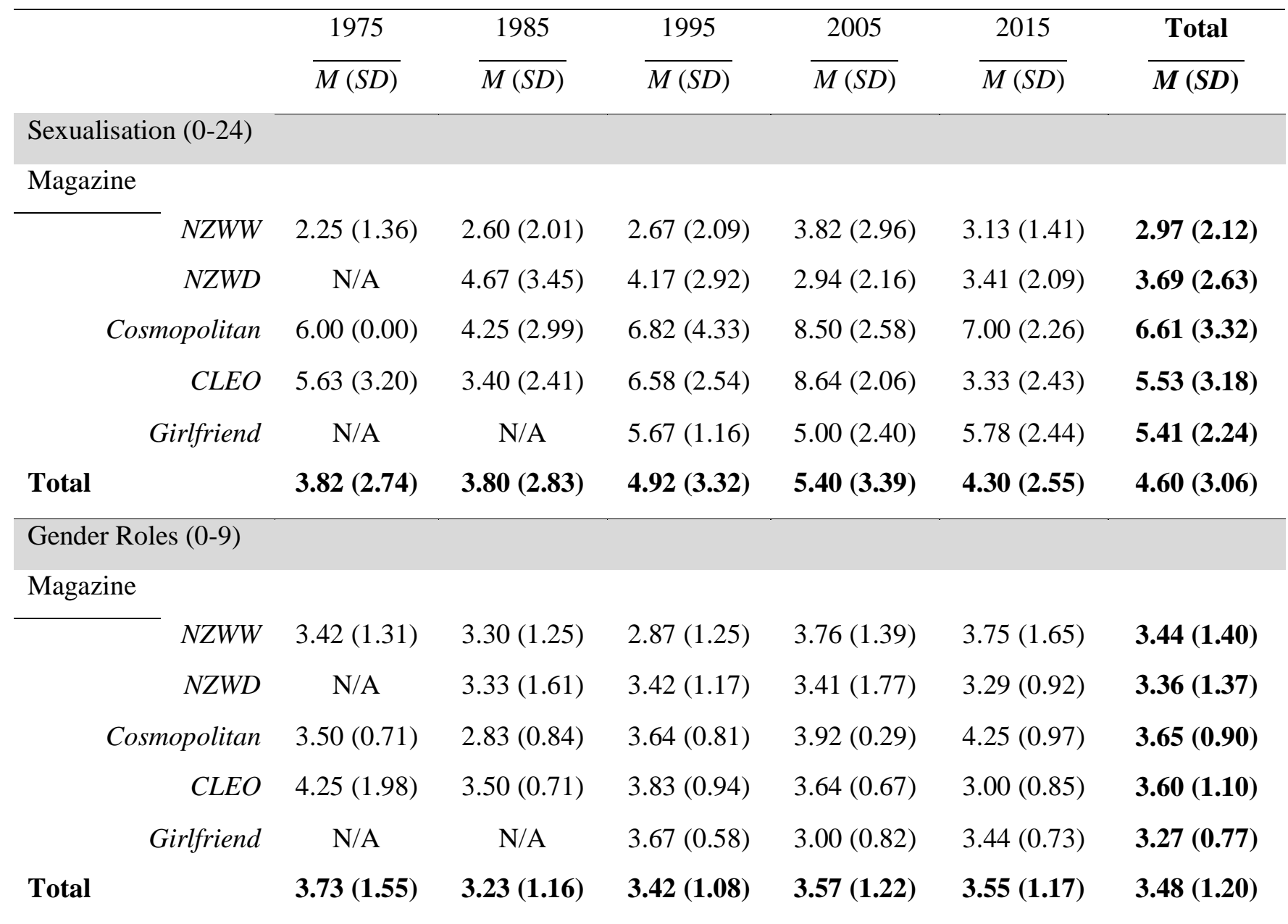

Note. $M$ indicates the mean score, $S D$ refers to the standard deviation.

\section{Inferential Statistics}

Differences in the dataset are noticeable in both quantitative and qualitative ways. The coding scheme was developed so that statistical tests could determine differences between groupings of magazine covers. However, one should not ignore the power of using visual examples for also illustrating significant differences. Therefore, alongside the statistical analyses that provide evidence of differences in my dataset, in this section I have also included examples of cover pages that illustrate the numerical differences.

\section{Sexualisation}

To examine the differences between the levels of sexualisation in the dataset, a oneway analysis of variance (ANOVA) was conducted. The initial ANOVA result indicated that there was a significant difference between year groups for sexualisation scores $F(4,247)=$ $2.64, p=.03$. A least-significant difference (LSD) post-hoc test was also computed as the 
overall ANOVA was significant and this allows for examination of how the groups differed (Field, 2009). The results of the LSD post-hoc test indicated that the sexualisation scores from cover pages published in $2005(M=5.40)$ differed significantly from those published in $1975(M=3.82), 1985(M=3.80)$ and 2015 ( $M=4.30)$. Namely, magazine covers published in the year 2005 featured images that were significantly more sexualised than covers from the year 1975, 1985 and 2015. There were no other significant interactions between year groups indicated in the post-hoc analysis. Based on previous literature a more linear pattern of increase over time (e.g. lowest score in 1975, highest in 2015) was expected from these interactions. However, this result offers some support for the second hypothesis of this study, that sexualisation would increase over time.

To highlight differences over time Figure 2 displays magazine covers that scored the average level of sexualisation for each year group ${ }^{12}$.

The cover pages from 1975 and 1985 both scored ' 4 ' on the sexualisation measure of the coding scheme. The image from 1975 scored ' 1 ' for touch as the model is casually holding an object, and ' 3 ' for facial expression as she is looking upwards at the viewer, smiling, with a tilted head. All other codes scored ' 0 '. The cover page from 1985 also scored a ' 4 ' in total for sexualisation. This cover scored ' 1 ' for clothing/nudity as the neckline of this dress is low and a modest amount of skin is revealed. Touch scored ' 1 ' as the woman is casually clasping her hands behind her back, facial expression also scored ' 1 ' as she is looking away from the viewer. Finally, the image also scored ' 1 ' on the head/body shot code as this is a full photo of the subject. All other codes scored ' 0 '.

The total score of the images presented from 1995 and 2005 was ' 5 '. The image from 1995 scored ' 1 ' on clothing/nudity as the models arms/shoulders are exposed. The image also scored ' 1 ' on mouth as her lips are slightly parted. Finally, this image also scored a ' 3 ' on facial expression as the model is looking up at the viewer, with a tilt to her head and a slight pout. The cover page from 2005 also scored ' 5 '. Clothing/nudity was scored as '2' because the arms and shoulders are revealed and the neckline was judged as slightly plunging. Touch also scored ' 1 ' because there is casual touch present between the two figures. Head/body shot also scored ' 1 ' as the majority of the woman's body is shown. Also text scored ' 1 ' as the image caption reads "Emily and Callum... sizzling!" which was judged to be slightly suggestive of sex/sexualisation.

\footnotetext{
${ }^{12}$ Mean scores for the magazines were rounded to the nearest whole number to select the example covers.
} 
The cover page from 2015 also scored '4' for total sexualisation. Clothing/nudity scored ' 2 ' as the shorts that are being worn in the image are revealing. Touch scored ' 1 ' as she has her hands casually on her own hips. Head/body shot also scored ' 1 ' as the image shows more of her body than just her head and shoulders.

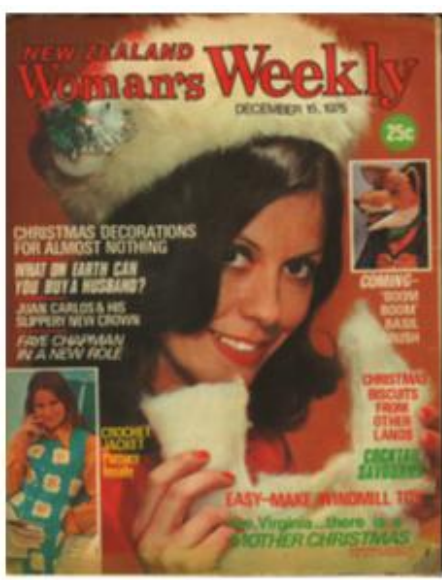

1975

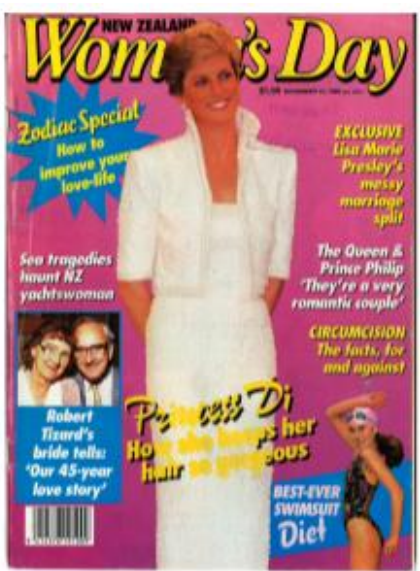

1985

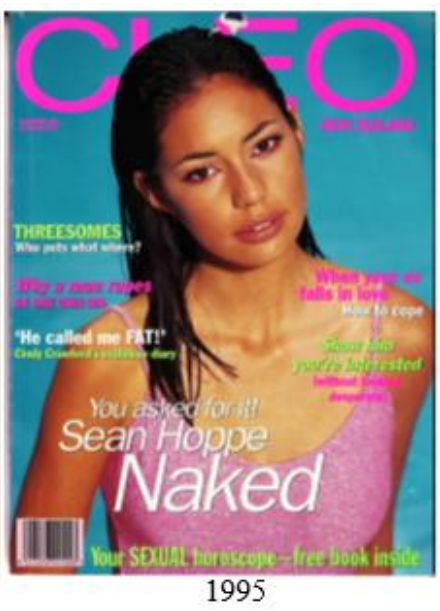

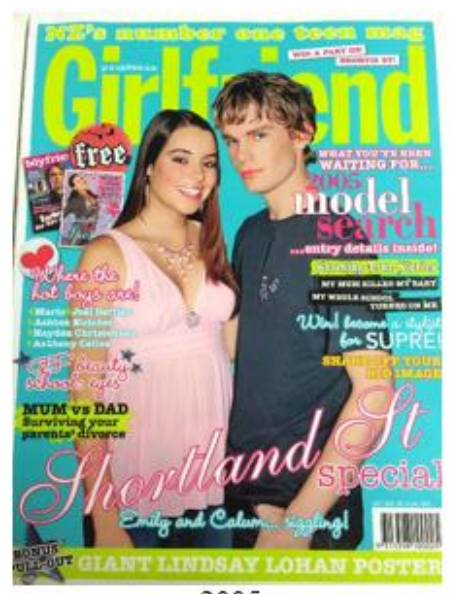

2005

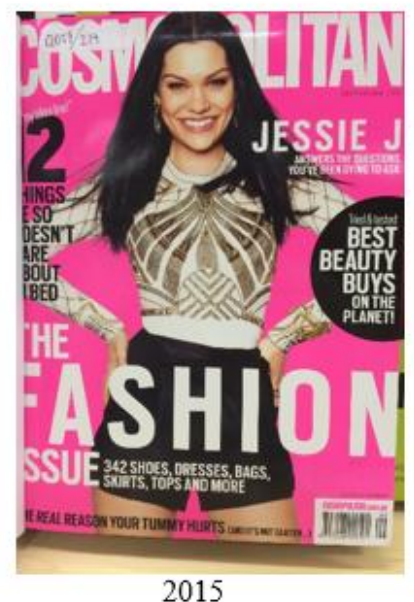

Figure 2. Example cover pages for mean scores across year groups.

1975: From New Zealand Woman's Weekly December $16^{\text {th }} 1975$. Copyright 1975 by Bauer Media Pty Limited. Reprinted with permission. 1985: From New Zealand Woman's Day November $15^{\text {th }} 1989$. Copyright 1989 by Bauer Media Pty Limited. Reprinted with permission. 1995: From CLEO November 1995. Copyright 1995 by Bauer Media Pty Limited. Reprinted with permission. 2005: From Girlfriend July 2005. Copyright 2005 by Pacific Magazines. Reprinted with permission. 2015: From Cosmopolitan Australia Septemeber 2015. Copyright 2015 by Bauer Media Pty Limited. Reprinted with permission.

These images help to illustrate the significant differences between images that may not appear, numerically, to be that large. Also on show is the importance of capturing overt and covert features of sexualisation - for example the image from 1995 and 2005 scored the same but the image from 1995 is more overt in its expression of sexualisation as the majority of the score comes from the facial expression code. On the other hand, the cover from 2005 scored ' 5 ' because of the combined effect of multiple low level activations of coding categories. 
The next stage in this analysis was to split the data file by magazine to examine whether any differences existed between the publications. There was a significant difference between magazines, $F(4,247)=16.32, p<.001$. The results of the LSD test showed that women on cover pages from Cosmopolitan $(M=6.61)$, CLEO $(M=5.53)$, and Girlfriend $(M=$ $5.41)$ were significantly more sexualised than those from $N Z W W(M=2.97)$ and $N Z W D(M=$ 3.69). Post-hoc tests also indicated that Cosmopolitan was significantly more sexualised than CLEO. Figure 3 contains example cover pages that scored the mean level of sexualisation for each magazine.

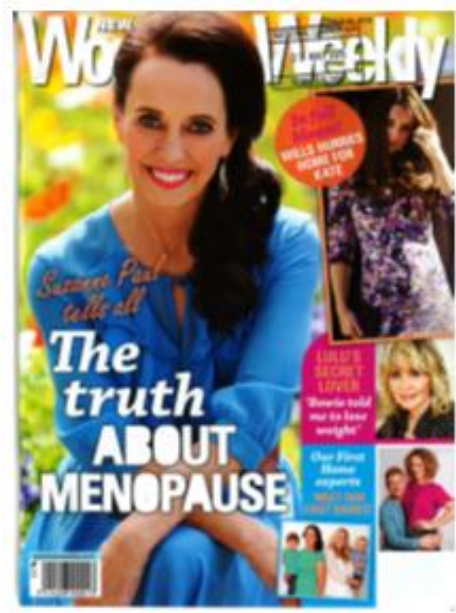

$N Z W W$

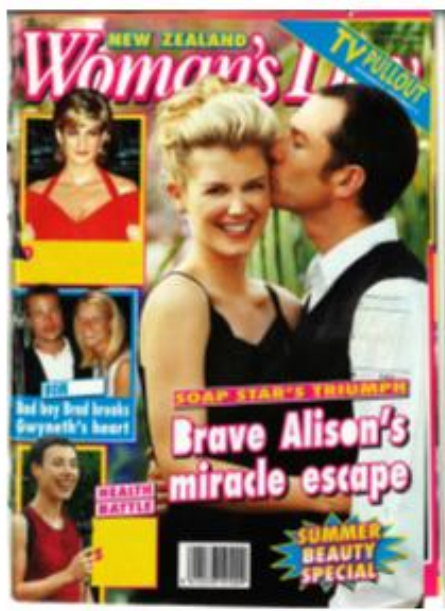

NZWD

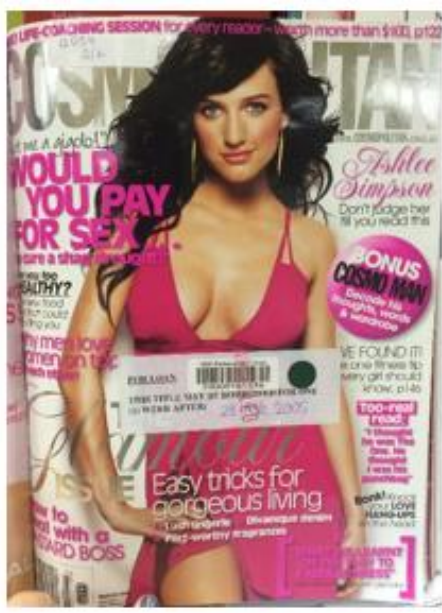

Cosmopolitan

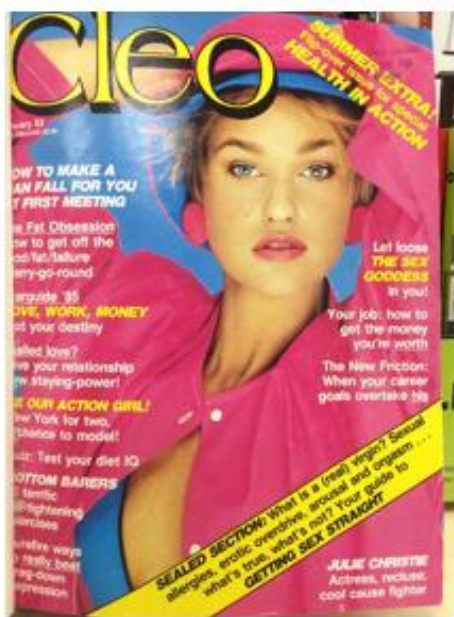

$C L E O$

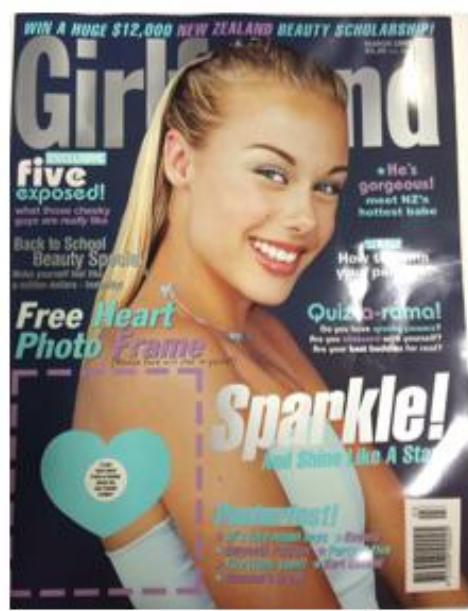

Girlfriend

Figure 3. Example cover pages for mean scores across publications.

NZWW: From New Zealand Woman's Weekly March $16^{\text {th }} 2015$. Copyright 2015 by Bauer Media Pty Limited. Reprinted with permission. NZWD: From New Zealand Woman's Day December $11^{\text {th }} 1995$. Copyright 1995 by Bauer Media Pty Limited. Reprinted with permission. Cosmopolitan: From Cosmopolitan Australia March 2005. Copyright 2005 by Bauer Media Pty Limited. Reprinted with permission. CLEO: From CLEO January 1985. Copyright 1985 by Bauer Media Pty Limited. Reprinted with permission. Girlfriend: From Girlfriend March 1999. Copyright 1999 by Pacific Magazines. Reprinted with permission. 
These example cover pages illustrate the differences between what the average sexualisation score looks like between magazines. The exemplar from $N Z W W$ has a score of ' 3 '. This publication had the lowest overall mean for sexualisation out of all the magazines. In the example image a score of ' 1 ' was given for 'touch' as the woman has her hands folded on her lap in a casual way. A score of ' 1 ' was also give for pose as she is sitting. Head/body shot was also scored as ' 1 ' because the majority of her body can be seen in the image.

The cover from $N Z W D$ was scored '4', a point was given for clothing/nudity as the dress worn by the woman reveals her arms and shoulders. Touch was scored as ' 2 ' because both figures have their arms around each other and the woman is being kissed on the cheek; these together are indicative of slightly sexualised behaviour. Head/body shot was also scored as ' 1 ' because again the majority of the woman's figure is included in the image.

The cover from Cosmopolitan scored ' 7 ', a significant jump in sexualisation from the average scores of $N Z W W, N Z W D$. In this image a score of ' 3 ' was given for clothing/nudity, this is because the outfit has a plunging neckline and is pulled up to also reveal the woman's thigh. Touch was scored ' 2 ' because the woman in the image is resting her hand on the top of her thigh, gathering the material of her skirt in the process, this was judged as being slightly sexually provocative. A score of ' 1 ' was also given to the breasts code because in this image the woman's breasts are a focal point, but they are still mostly covered. Head/body shot was also scored ' 1 ' because the shot encompasses more than just the woman's head and shoulders.

The example cover page from CLEO scored ' 6 ', this is again a significantly higher sexualisation score than that of $N Z W W$ and $N Z W D$. Clothing/nudity scored ' 3 ' as the model is wearing a top with a plunging neckline beneath her unbuttoned jacket. Touch was scored as ' 1 ' as her hands are clasped behind her head, pose was also scored as ' 1 ' because the model is lifting her arms behind her head without specific purpose. Mouth was also scored as ' 1 ' because the model has her lips slightly parted and is pouting slightly.

The cover page from Girlfriend scored a total of ' 5 ' on the sexualisation part of the coding scheme, an average score that is significantly higher than those from $N Z W W$ and $N Z W D$. One point was assigned for clothing/nudity as the model's arms and shoulders are revealed in a modest way. Touch was also scored as ' 1 ' as the image implies the model has her hands clasped in front of her. Facial expression was scored as ' 3 ' because the model is smiling, with her head tilted, looking up at the viewer.

These examples illustrate the differences between publications beyond the numbers. Cover pages from Cosmopolitan, CLEO, and Girlfriend are at first sight different from the 


\section{LOOK AT HER: WOMEN IN WOMEN'S MAGAZINES}

images used on the covers of $N Z W W$ and $N Z W D$. The results from this study provide empirical evidence towards the idea that these differences are related to the level of sexualisation within the images.

Following this analysis, the next step was to split the data file by magazine and test if the interaction between year group and sexualisation scores was still present. The results indicated that there were significant differences between year group and sexualisation scores in just Cosmopolitan $(F(4,44)=2.99, p=.029)$ and $C L E O(F(4,48)=8.84, p<.001)$. The results of the LSD test indicated that for Cosmopolitan magazine covers from 2005 ( $M=8.50)$ and $2015(M=7.00)$ were significantly more sexualised than those from $1985(M=4.25)$. For CLEO magazine the post-hoc results indicated that covers from $1995(M=6.58)$ were significantly more sexualised than covers from both $1985(M=3.40)$ and $2015(M=3.33)$. Also, CLEO covers from $2005(M=8.64)$ were significantly more sexualised than those from $1975(M=5.63), 1985(M=3.40)$ and $2015(M=3.33)$.

Again, example cover pages from Cosmopolitan and CLEO that scored the average level of sexualisation for these year groups illustrate the differences beyond their numerical value. Figure 4 shows example covers from Cosmopolitan for the higher sexualised years (2005 and 2015) and the lowest sexualised year (1985). The cover image from Cosmopolitan 1985 scored ' 4 ': one point came from the clothing/nudity code as beneath the jacket you can see that the neckline is rather low, the other 3 points came from the facial expression code as the model is looking up, with head slightly tilted and lips slightly pouted at the viewer. In contrast to this relatively low level of sexualisation, the covers from 2005 and 2015 scored ' 9 ' and ' 7 ' respectively. The cover from 2005 scored ' 3 ' on clothing/nudity; '2' on touch for the placement of her hands (on upper thigh and chest); ' 1 ' for pose (hip cocked with leg open), ' 1 ' for mouth (slightly parted); ' 1 ' from breasts (suggestive of exposure by hand placement and straining of the single button on the shirt); and ' 1 ' for head/body shot. The cover from 2015 scored ' 2 ' for clothing/nudity (the neckline is low and the skirt is high on the thigh); ' 1 ' for touch (casual placement of hand on ankle); ' 1 ' for pose (sitting/kneeling); ' 1 ' for head/body shot; and ' 2 ' for text as the caption reads "50 Shades' Dakota Johnson: the girl who shot those sex scenes" which is a direct reference to sex. 


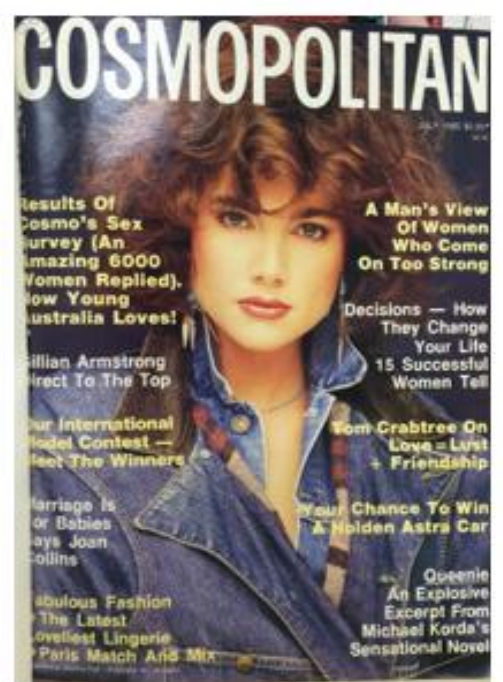

1985

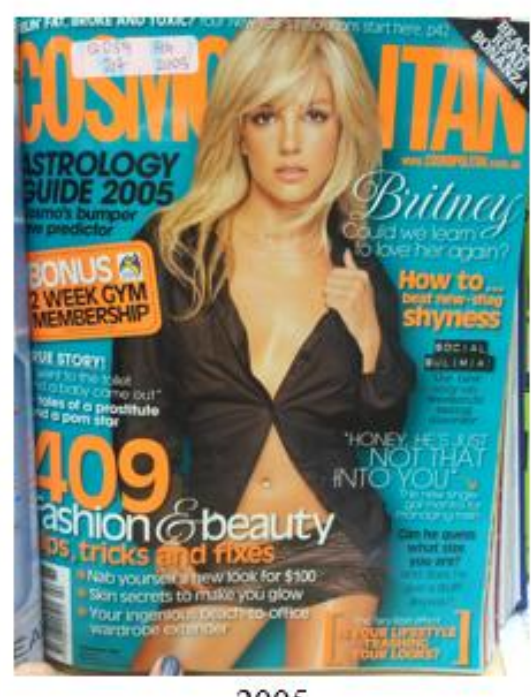

2005

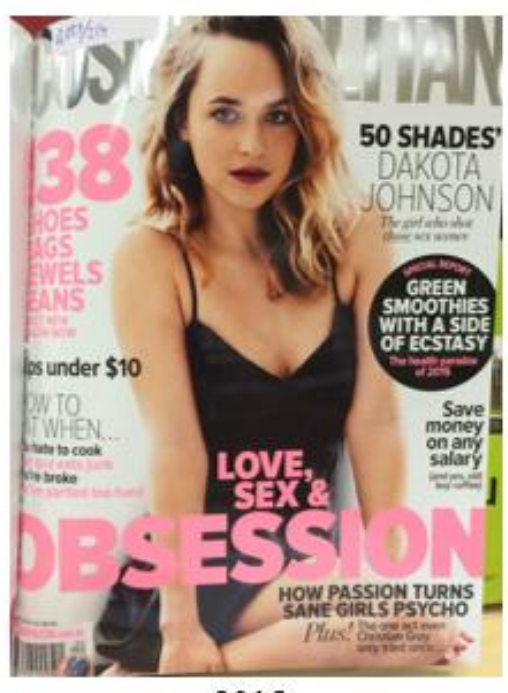

2015

Figure 4. Example Cosmopolitan cover pages with average sexualisation scores from 1985, 2005, and 2015.

1985: From Cosmopolitan Australia July 1985. Copyright 1985 by Bauer Media Pty Limited. Reprinted with permission. 2005: From Cosmopolitan Australia February 2005. Copyright 2005 by Bauer Media Pty Limited. Reprinted with permission. 2015: From Cosmopolitan Australia April 2015. Copyright 2015 by Bauer Media Pty Limited. Reprinted with permission.

The difference between these images is not only apparent numerically but also from visual inspection. Whilst the model's face from the 1985 cover page is overt in its allure, you can see that the images selected for 2005 and 2015 are carefully positioned to convey sexuality in heightened ways. These images are not candid shots that one might see recreated in a normal setting; they are crafted in specific ways, some of which convey heightened sexual objectification of their subjects. 


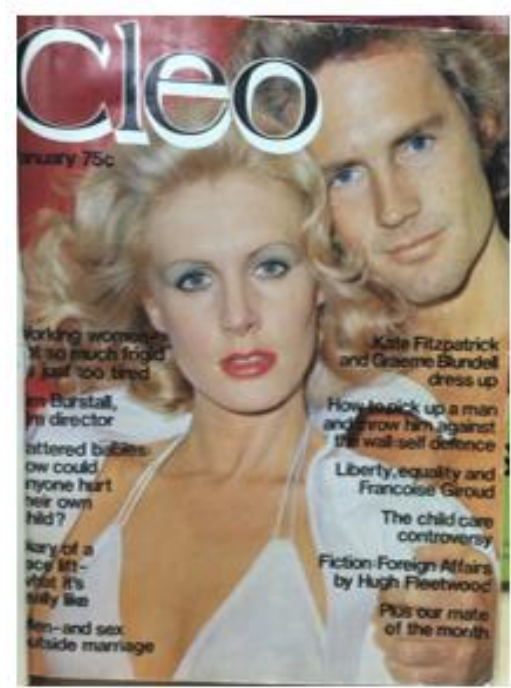

1975

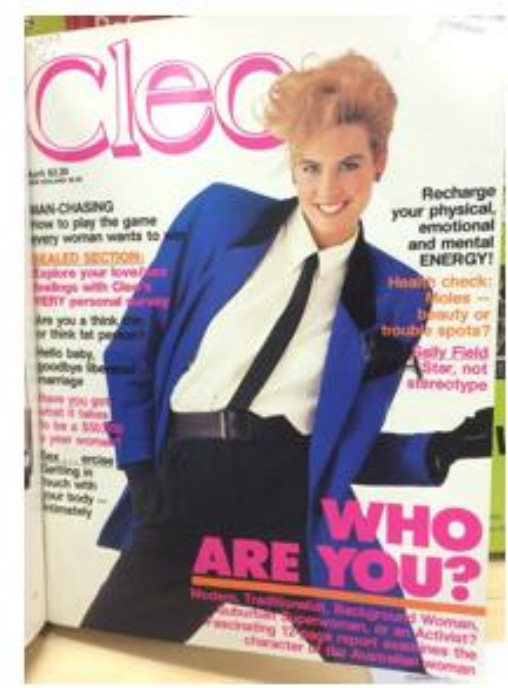

1985

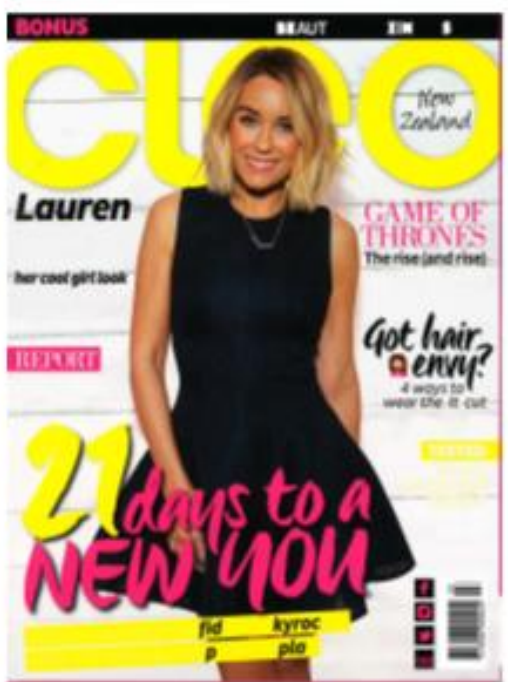

2015

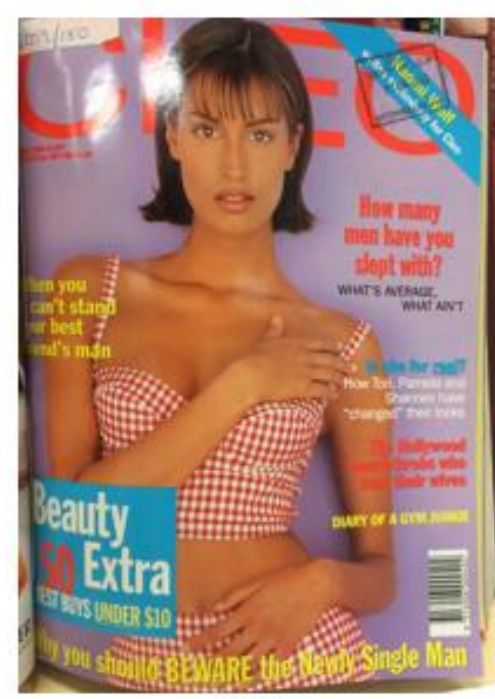

1995

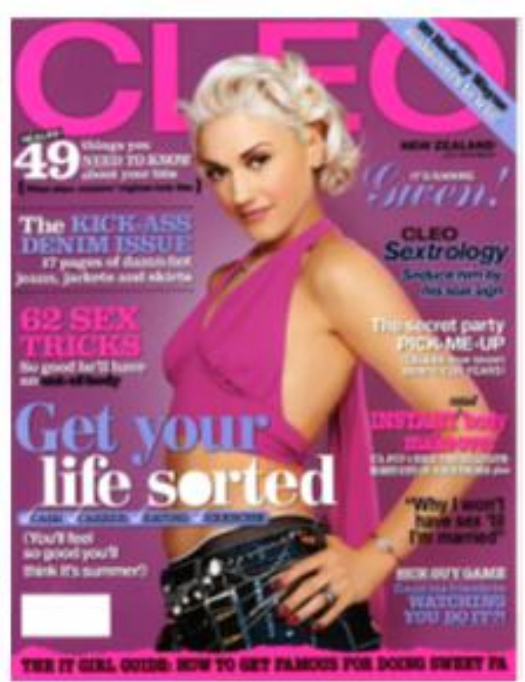

2005

Figure 5. Example CLEO cover pages with average sexualisation scores from 1975, 1985, 1995, 2005, and 2015.

1975: From CLEO January 1975. Copyright 1975 by Bauer Media Pty Limited. Reprinted with permission. 1985: From CLEO April 1985. Copyright 1985 by Bauer Media Pty Limited. Reprinted with permission. 2015: From CLEO March 2015. Copyright 2015 by Bauer Media Pty Limited. Reprinted with permission. 1995: From CLEO March 1995. Copyright 1995 by Bauer Media Pty Limited. Reprinted with permission. 2005: From CLEO July 2005. Copyright 2005 by Bauer Media Pty Limited. Reprinted with permission.

Figure 5 shows example cover pages from $C L E O$ for the lowest sexualised years (1975, 1985 and 2015) and highest sexualised years (1995 and 2005). The lower scoring images from 1975, 1985 and 2015 scored '6', '3', and '3' respectively. The image from 1975 scored ' 3 ': for clothing/nudity (plunging neckline); ' 1 ' for touch (of the male model on the woman's upper arm); ' 1 ' for mouth (slightly parted); ' 1 ' for text as the image caption reads "Kate Fitzpatrick and James Blundell dress up" which was judged to be a slight innuendo. The cover page from 1985 scored '3': one point from touch (casual); one point from pose 
(leaning), and ' 1 ' for head/body shot. Likewise, the cover from 2015 scored '3', one point from clothing/nudity (exposed arms); one point for touch (casual); and one point for head/body shot.

The higher scoring years, 1995 and 2005, scored ' 7 ' and ' 8 '13 respectively. The image from 1995 scored ' 2 ' for clothing/nudity (visible midriff); '2' for touch as the left hand is in a position indicative of clothing removal; ' 1 ' for mouth (slightly parted); ' 1 ' for breasts as the hand positioning is indicative of exposure; and ' 1 ' for head/body shot. The cover from 2005 scored '2' for clothing/nudity (visible midriff); ' 1 ' for touch (casual); ' 1 ' for pose (leaning backwards rather than standing straight); ' 3 ' for facial expression (looking up at viewer, head tilted); and ' 1 ' for head/body shot.

As with the examples from Cosmopolitan, the images from CLEO in the higher scoring years seems to be deliberately staged for sexual objectification. The implications of these differences are discussed in more detail in the next chapter.

Non-significant differences were found when raw sexualisation scores were compared across seasons and solo/group gender make-up. However, when the data-set was split by magazine there was a significant difference in sexualisation levels across gender make-up for $N Z W W$ covers, $F(2,67)=3.74, p=.029$. LSD tests indicated that for $N Z W W$ covers with a mixed gender group were more sexualised than covers depicting a woman by herself. Solo/group image and gender make-up were demographic variables collected within the coding scheme. They refer to whether the image on the cover is of a solo person or a group and in the event of a group photo which genders are represented in the image.

\section{Gender Roles}

ANOVA tests were also used to examine any differences between gender role scores across year groups and other variables. When data was compared across year groups, magazines, and seasons no significant differences were found. However, when data was split by magazine and then compared across year groups significant differences were found for Cosmopolitan magazine, $F(4,44)=5.62, p=.001$. Post-hoc LSD tests indicated that these differences are between $1985(M=2.83)$ and $1995(M=3.64), 2005(M=3.92)$, and $2015(M=$ 4.25). The results indicate that less traditional gender roles were depicted in covers from 1985 compared to those from 1995, 2005, and 2015.

\footnotetext{
${ }^{13}$ The rounded average score for 2005 was actually 9 but no covers from this year were scored exactly 9 on the sexualisation measure. This cover scored 8 and is the closest representation of the mean score from within the sample.
} 


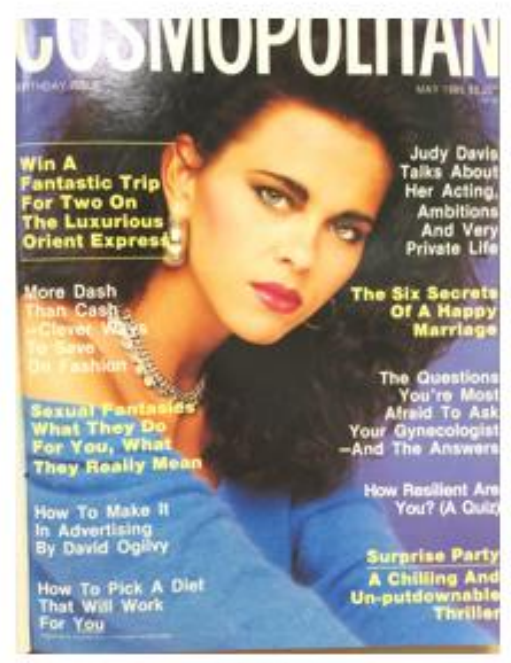

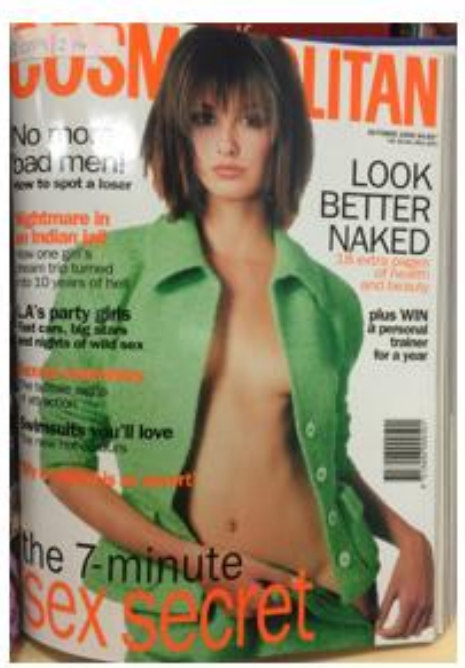

1995

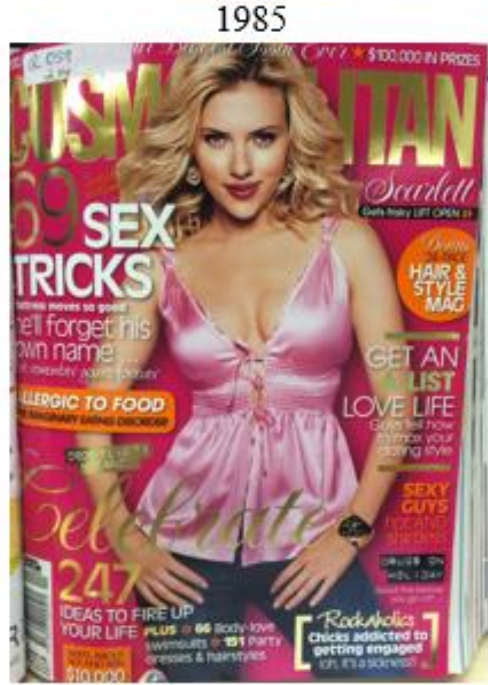

2005

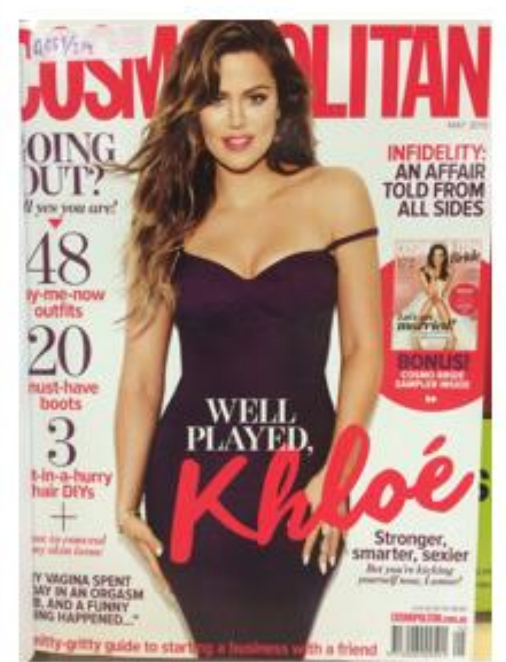

2015

Figure 6. Example Cosmopolitan cover pages with average gender role scores from 1985, 1995, 2005, and 2015.

1985: From Cosmopolitan Australia May 1985. Copyright 1985 by Bauer Media Pty Limited. Reprinted with permission. 1995: From Cosmopolitan Australia October 1995. Copyright 1995 by Bauer Media Pty Limited. Reprinted with permission. 2005: From Cosmopolitan Australia November 2005. Copyright 2005 by Bauer Media Pty Limited. Reprinted with permission. 2015: From Cosmopolitan Australia May 2015. Copyright 2015 by Bauer Media Pty Limited. Reprinted with permission.

Figure 6 provides examples of cover pages that had the average gender role score for each of the year groups. The lower scoring cover (1985) has a score of ' 3 ' and the higher scoring covers $(1995,2005$, and 2015) all have a score of ' 4 '. For the cover from 1985 the codes present are: ritualisation of subordination, as the model is sitting and hunched over, therefore lowering/lessening her physical presence; location, as the model is in an unidentifiable location; and objectification as the model has no purpose in this image aside from being looked at. The covers from 1995, 2005, and 2015 all have the same codes present. Feminine touch - in the images from 2005 and 2015 this is because the women in the images 
are touching themselves in a soft, gentle manner as if they were caressing themselves in some way. In the image from 1995 'feminine touch' was judged as present because the model is removing her skirt in a way that is for show rather than how one would practically unzip a skirt. All three images also scored a point for body display as the women are dressed in revealing (by lack of coverage or tightness of the outfit) clothing. All three images also have indiscernible locations, and all the women have no purpose in the images aside from being viewed.

Finding that gender role scores differed significantly from 1985 compared to 1995 , 2005 and 2015 is contrary to the fourth hypothesis made for this research. It was predicted that there would be no overall change in gender role scores. It is very interesting that 1985 shows a drop in the depiction of traditional gender roles, this will be discussed further in the next chapter.

Gender role scores were also compared across group/gender make up. An initial significant ANOVA result $(F(2,249)=45.66, p<.001)$ and LSD post-hoc results indicated that higher gender role scores were to be expected in covers where there was a mixed gender group compared to both solo female covers and groups made up entirely of women. Given two of the codes for the gender roles coding scheme cannot be 'scored' without the presence of a male, this result is unsurprising. Indeed, when scores from these two codes ("relative size" and "function ranking") were controlled, the difference between the two groups was non-significant.

\section{Summary}

The data shows that sexualisation scores were relatively low in the overall sample of magazines. Comparisons between year groups indicated that images of women from 2005 were significantly more sexualised than those from 1975, 1985, and 2015. Analyses between publications indicated that women on the cover pages of Cosmopolitan, CLEO, and Girlfriend were significantly more sexualised than those on the covers of both $N Z W W$ and NZWD. Cosmopolitan also had significantly higher levels of sexualisation than CLEO. Examining year group differences within each publication indicated significant differences only for Cosmopolitan and CLEO. In Cosmopolitan, images of women were significantly more sexualised in cover pages from 2005 and 2015 than those from 1985. In CLEO, images from 1995 and 2005 were more sexualised than those from 1985 and 2015. Also images from CLEO in 2005 were more sexualised than those from 1975. Differences are described numerically and illustrated through the use of examples from the sample. 


\section{LOOK AT HER: WOMEN IN WOMEN'S MAGAZINES}

Gender role average scores indicated that the depiction of gender roles in the present sample were at the mid-point on the scale. No significant differences were found when data were compared by year group or magazine. When the data was split by magazine and then compared by year group, only Cosmopolitan showed a significant difference with images of women from 1985 showing less traditional gender role depiction than cover page images from 1995, 2005, and 2015. This was highlighted by example cover pages. Despite these nonsignificant comparisons, frequency data when the coding scheme was split into each individual code showed some interesting patterns that will be discussed in further detail in the next chapter. 


\section{Chapter Five: Discussion}

This chapter comprises a discussion that locates the key findings within a wider context, suggests potential implications and applications of the results.

To recap, the purpose of this thesis was to provide an analysis of the depiction of women on the cover pages of women's magazines across a 40 year time period in a New Zealand context. Findings showed that on average the level of sexualisation of women, in relation to the scale (maximum score 24) is relatively low. However, across year groups and magazine types there are interesting fluctuations that will be discussed in further detail. Analyses on the depiction of traditional gender roles indicate little variability over time and magazine with average scores sitting near the mid-point on the scale. Whilst parametric analyses found few significant differences in this half of the dataset some interesting patterns were found in the frequency data which will be discussed in more detail in this chapter.

\section{The Findings in Context}

\section{Demographic Variables}

Frequency data collected from the magazine covers in this sample indicate overwhelmingly that women represented in these images are likely to be NZ European (83.3\%), aged 18-35 (73.0\%). Firstly, this highlights that the women depicted on the cover of magazines are not representative of all women in New Zealand society (Statistics New Zealand, 2013). Census data tells us that the median age for women in New Zealand is 38.9 years. The data shows that $73.0 \%$ of women depicted in cover images are below this median age which reinforces the idea that youthfulness is tied to the image of the 'ideal' woman. In addition to this, census data also shows that $74.0 \%$ of the population identify as NZ European, $14.9 \%$ as Maori, $11.8 \%$ as Asian, and $7.4 \%$ as a Pacific person (Statistics New Zealand, 2013). The current results therefore have an overrepresentation of those who could be deemed New Zealand European and an underrepresentation of other ethnicities that have a significant presence in New Zealand society. These overrepresentations are important to highlight, because image selection within a publication (as discussed earlier) is not a random process: those who select these images do so with the intention to entice consumers and sell the idea that the magazine could help the reader achieve this 'ideal' (McCracken, 1993; Sonnevend, 2012).

The saturation of young, NZ European women within this sample of cover pages could therefore be a further reflection of the normalisation of the 'ideal'. This choice is in line 


\section{LOOK AT HER: WOMEN IN WOMEN'S MAGAZINES}

with other research that has shown this is the 'type' of woman presented in women's media (Haboush et al., 2012). Standardised images of women can be harmful because even with acknowledgement that this 'ideal' is unrealistic, it is still a point of comparison for women (Richins, 1991). This can be a trigger for self-objectification within women who do not fit these narrow criteria (Frederick, Rorbes, Grigorian \& Jarcho, 2007). Women's magazines have carved out a section of the media market by constructing themselves as tableaus for dispensing information about how to be a woman and what it means to be a woman (Burton, 2005; Croteau \& Hoynes, 2014). The current findings provide evidence in the demographic variables of the chosen cover figures that women's magazines can perpetuate harmful ideals.

An interesting extension of these demographic findings would be to statistically analyse sexualisation and gender role scores between demographic variables (such as age or ethnicity of the cover figure). The current research was unable to do this because the severely skewed sample sizes (see Table 4) would have hindered the ability to calculate reliable statistics (Field, 2009). This prevents an intersectional analysis of the sexualisation and depiction of traditional gender roles in images of women between different age and ethnic groups. Intersectionality refers to the connections between all forms of inequality and how each form is influenced by the others (Collins, 2015). Gender inequality can therefore be experienced differently within different races, classes, ages, sexualities, ethnicities, nations, and abilities (Collins, 2015). The inability to analyse the dataset across demographic boundaries mean that inferences and conclusions drawn within this thesis are limited. The generalisability of inferences from this research is restricted as studies have shown that the impact sexualised images have is affected by variables such as the cohesive age or ethnicities between the subject of the image and the viewer (Haboush et al., 2012).

Research has shown that there are differences in the depiction of women between ethnicities (Baker, 2005). A comparative analysis looked at media for White women and men and for Black women and men and found that White women were objectified more than Black women in their respective media forms. As well as this, White women were portrayed as more submissive and dependant compared to Black women (Baker, 2005). This suggests that portrayals of sexual objectification and gender roles could be different depending on the potential audience and ethnicity of the image subject. In addition to this, research has also shown that age is a protective factor against self-objectification. It was found that as age increased levels of self-objectification decreased (Tiggemann \& Lynch, 2001). Viewing objectifying/sexualising images and comparing oneself to them is a key part of the process of self-objectification (Frederickson \& Roberts, 1997). It could potentially be inferred that the 


\section{LOOK AT HER: WOMEN IN WOMEN'S MAGAZINES}

decrease in self-objectification with age might be related to a decrease in sexually objectifying images of that age group. A comparison of the level of sexualisation across age and ethnicity of the subject of the cover image would have been a starting point for an analysis that explored this idea further. Not being able to explore the potential effects of these due to the skewed sample size in greater detail is a limitation of the current dataset.

\section{Sexualisation}

In this section the findings from the analysis of the sexualisation part of the coding schedule will be discussed. The level of sexualisation in images on the cover pages of women's magazines was important to investigate because the sexualisation of women can contribute to rape culture, and normalising sexual objectification within women's media sets a dangerous precedent for women. This section will discuss the overall mean scores, differences across year groups, and differences across magazines with reference to previous literature and the hypotheses made at the end of the second chapter. Further inferences about these results are detailed in the implications section of this chapter.

The sexualisation scale developed for this research focussed on key features of how women are sexualised in magazine images in ways identified in previous literature (Bell, 2001; Hatton \& Trautner, 2011). The scale had ten coding categories and scores could range from a minimum of ' 0 ' to a maximum of ' 24 '. With the results from the current study it is important to firstly acknowledge that the average sexualisation scores across the sample of magazine covers were at the lower end of the scale. However, the analytic strategy used in Hatton and Trautner's (2011) study shows that whilst low, some of the mean scores from the current study indicate that the covers were still sexualised. Hatton and Trautner (2011) grouped covers into non-sexualised (scores of 0-4), sexualised (scores of 5-9), and hypersexualised (scores of 10+) groups. The logic behind these divisions is that within their research, the cut-off point between each category signified a change in the impact of the cover images. Those in the non-sexualised category were innocuous, those in the sexualised category presented a subtle level of sexuality, and the hyper-sexualised category exhibited an overt display of sexuality. This analytic strategy was not utilised in the current study because grouping covers this way would have resulted in skewed groups sizes that may have impacted the validity of the overall results. However, as the current coding scheme and overall methodologies are very similar, some tentative inferences can be made. If Hatton and Trautner's (2011) categories are used as a framework then some of the mean scores from the present study would fall into the sexualised category, namely, average scores for cover pages 
from the year groups 1995, 2005, and those from Cosmopolitan, CLEO, and Girlfriend magazine. Mean scores for 1975, 1985 and the publications NZWW and NZWD would fall into Hatton and Trautner's (2011) non-sexualised category.

Based on the framework from this comparable study, the overall mean score for sexualisation (4.60) straddles the boundary between non-sexualised and sexualised. This suggests that while scores are low on the scale, this should not be considered indicative of an absence of sexualisation. Referring back to Figure 1, the example cover pages illustrate this difference. The example cover pages from 1975, 1985, and 2015 in Figure 1 all score '4' which place them in Hatton and Trautner's (2011) non-sexualised group. A score of '4' places these images at the higher end of this category and there are elements in these images that suggest sexualisation. However, when compared to the cover pages from 1995 and 2005 (which scored ' 5 ', meaning they would be in Hatton and Trautner's (2011) sexualised group) the difference is small but significant - this is noticeable from just an initial impression. The sense that an invisible boundary from non-sexualised to sexualised has been crossed is palpable in these images. The implications of these low level changes in sexualisation are discussed further in a later section of this chapter.

The first hypothesis of this study predicted that women would be depicted in a sexualised manner within cover page images. The descriptive statistics indicate that this is somewhat correct. There is a residual, low level of sexualisation throughout the cover page images in this research.

The statistical analyses indicate that there are several interesting significant differences between sexualisation scores in the data. The first difference directly relates to the second hypothesis made in the study, predicting that sexualisation scores would increase across the time period examined (1975-2015). ANOVA results indicate that this prediction was not correct, with the pattern of fluctuation being more complex than a simple linear increase. It was found that images of women from 2005 were the most sexualised and images from 1985 were the least sexualised. Sexualisation scores from 2005 were significantly larger than those from 1975, 1985, and 2015. There were no other significant differences between year groups. This, along with mean scores, indicated that rather than a gradual increase over time there is a peak in the data in what otherwise would have been a differing, but not significantly changeable, dataset (when compared across years).

This result has both similarities and differences to past research. The early work by Soley and Kurzbad (1986) indicated that sexualised portrayals of women were relatively commonplace in their analysis of advertisements from 1964 and 1984. Their study was 
angled towards addressing the prevalence of sexualisation rather than a content analysis of how women are sexualised. However, their findings do indicate that the use of visual sexualisation increased between the two time periods in their study with prevalence figures rising from $69.9 \%$ in 1964 to $79.1 \%$ in 1984 (Soley \& Kurzbad, 1986). This result indicates a rising prevalence of sexualisation amid publications. The current results differ from this in that there are fluctuations across year groups with a distinct peak. Of course, Soley and Kurzbad (1986) had a different focus and year groups so the current results are not directly comparable. One feature to note is the proportion of sexualised advertisements that was found in their 1984 group. Soley and Kurzbad (1986) found that 79.1\% of advertisements were visually sexualising towards women from publications printed in 1984. The results of the current study indicate that 1985 had the lowest sexualisation scores for cover page images which is contrary to the increase in the amount of sexualised images found by Soley and Kurzbad (1986). This could indicate that there is a difference in the depiction of women within different sorts of images within a single publication. This presents an avenue for future research which will be discussed further in the next chapter.

The study by Reichert et al. (1999) found results that support those found in the current research. Reichert et al. (1999), like Soley and Kurzbad (1986), examined the level of sexualisation within advertisements across two distinct time periods (1983 and 1993). They found that the images from 1993 were more sexually explicit than those from 1983. The current results show a similar pattern for the results from 1985 and 1995 although the difference in this sample is not significant. The similarities in this trend are important as it shows that although my results are not quite what was expected, there is literature that has found similar trends. However, as the research by Reichert et al. (1999) does not cover the time period of specific interest in the current research (2005), it is hard to draw further inferences from this piece.

The most comparable study to the current research is that by Hatton and Trautner (2011). In this longitudinal analysis of the cover pages of Rolling Stone magazine the authors looked at the sexualisation of women from 1967 to 2009. Their results indicate, in line with the current study, that cover images from the 2000s are the most sexualised. Interestingly, Hatton and Trautner (2011) found that sexualisation increased dramatically from the 1980s to the 1990s with a small increase into the 2000s. In the current study the increase happened at a more gradual pace (as indicated by the non-significant difference for the time periods 1985 1995, and 1995 - 2005) which resulted in the peak year for significant differences in 2005. This difference is interesting because of the comparisons made in this previous literature 


\section{LOOK AT HER: WOMEN IN WOMEN'S MAGAZINES}

between gendered publications and general interest magazines. The research by Soley and Kurzbad (1986) and Reichert et al. (1999) found that women's magazines had higher levels of sexualised images (within advertisements) than general interest magazines did. Hatton and Trautner (2011) have found that within a general interest magazine (Rolling Stone) there was a sharp increase in sexualised, and hyper-sexualised images. The current results indicate that within women's magazines that over time increases have been gradual. Considering that Hatton and Trautner (2011) also found that in the 2000s hyper-sexualised images of women (those scoring over 10 on the coding schedules) made up $60 \%$ of the sample, it can be inferred that this general interest publication had significantly greater levels of sexualisation than the gendered magazines examined by current research. This is curious because it goes against the pattern established by previous literature.

No hypothesis was made about the difference in sexualisation levels between publications. However, the dataset showed some interesting significant differences. The results of this study showed that depictions of women on the cover pages of Cosmopolitan, CLEO, and Girlfriend magazines were all significantly more sexualised than those from $N Z W W$ and NZWD. As well as this, Cosmopolitan images were significantly more sexualised than cover pages from $C L E O$. The example cover pages in Figure 2 illustrate this. The images from Cosmopolitan, CLEO and Girlfriend give the impression that they are more sexual than those from $N Z W W$ and $N Z W D$. These results will probably not be surprising to anyone who has seen a copy of $N Z W W$ sitting next to Cosmopolitan while they have been standing in line at a supermarket checkout (see Figure 7). However, the numbers implore the question - why is it that these monthly 'glossies' have markedly more sexualised depictions of women on the cover? 


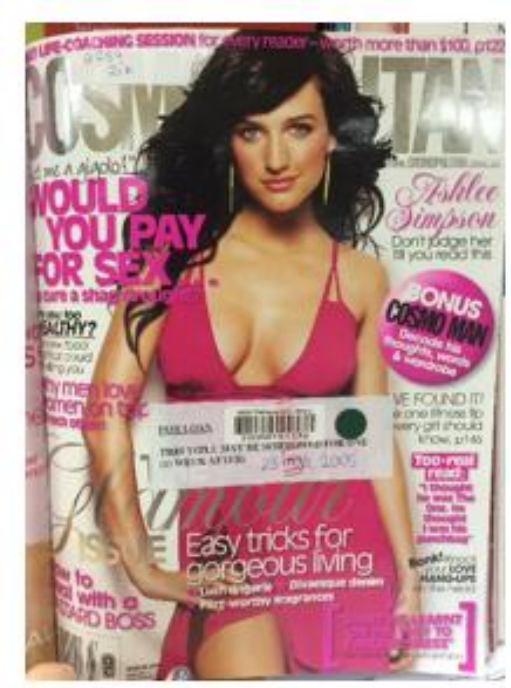

Cosmopolitan sexualisation score 7

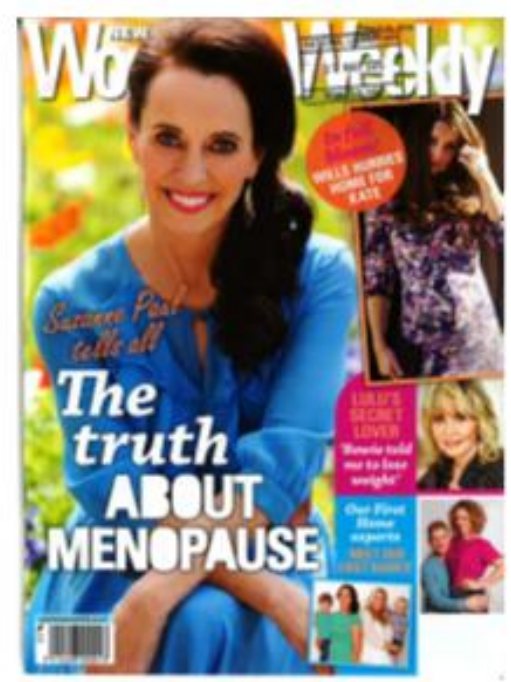

$N Z W W$ sexualisation score 3

Figure 7. Comparison of average sexualisation scores from Cosmopolitan and NZWW.

Left: From Cosmopolitan Australia March 2005. Copyright 2005 by Bauer Media Pty Limited. Reprinted with permission. Right: From New Zealand Woman's Weekly March $16^{\text {th }} 2015$. Copyright 2015 by Bauer Media Pty Limited. Reprinted with permission.

Women's magazines are not created equal, and all have their own niches within the market. Cosmopolitan, CLEO and Girlfriend market themselves on being "a girl's best friend" and pride themselves on pushing the boundaries within women's publications (Bauer Media New Zealand, 2015; Bauer Media Australia, 2016; Pacific Magazines, 2016). NZWW and NZWD, on the other hand, have carved out a section of the New Zealand market as the everyday (or every week) staples in women's lives: they are the traditional publications that offer material that is meant to offer relaxation and not challenge readers (Bauer Media New Zealand, 2016a, 2016b). In tandem with these publication blurbs the results of the current study suggest that heightened sexualisation may be being twinned with empowerment and independence within the monthly 'glossies'.

Both the descriptive and parametric results from the sexualisation section of the analyses show interesting trends both numerically and from visual inspection of example cover pages. These results show both similarities and differences to previous literature and the implications of this will be discussed further in a later section of this chapter.

\section{Gender Roles}

The depiction of gender roles was important to examine with women's magazines because of the explicit connections between gender inequality and sexual violence (Yodanis, 2004). Images that are specifically selected for magazine covers are not innocuous, as they 


\section{LOOK AT HER: WOMEN IN WOMEN'S MAGAZINES}

can reflect ideas about their subjects that are reflective of wider societal ideas (Burton, 2005; Carrabine, 2012). Therefore investigating whether traditional gender roles are reinforced for women in women's magazines is important because, as with sexualisation, normalising ideas that can facilitate sexual violence sets a concerning precedent. The depiction of traditional gender roles were examined individually by looking at frequency data and holistically by creating an additive scale that resulted in a total score for each cover page in the sample. The coding schedule had nine coding categories and scores could range from ' 0 ' to ' 9 ' on the overall scale. This section expands on the results reported in the previous chapter. Wider implications of these findings are discussed in a later section of this chapter.

Parametric testing for this part of the coding schedule produced mostly nonsignificant results. This is indicative of a consistent level of gender role stereotyping within cover page images across the publication. Average scores indicate that features of traditional gender roles were not overt but, as with sexualisation, have a regular subtle presence within cover page images. The lack of variation over the year groups is consistent with previous literature as well as the third and fourth hypotheses of the current study. The exception to this pattern is the results across year groups from when the dataset was split by magazine. The results of this analysis showed that in 1985 Cosmopolitan magazine covers had significantly fewer stereotypical depictions of gender roles than those published in 1995, 2005, and 2015. These differences are illustrated in Figure 3 where the cover page image from 1985 (score of '3') when compared to those from 1995, 2005 and 2015 (scores of '4') appears less like a generic, contrived image of a woman. The results from within Cosmopolitan are interesting because the fluctuations deviate from the rest of the dataset and also from the trends in previous literature.

Previous literature found that the overall presence of stereotypical gender roles did not change across the time periods they investigated. Sullivan and O'Connor (1988), studied and compared gender roles within advertisements from the 1950s, 1960s, and 1983. Within this previous study, it was found that women in advertisements from 1983 were depicted as more independent and as more versatile members of the workforce. This is arguably the impression that the example cover pages in Figure 5 convey. Images from 1985 were more individualistic and personal where as those from 1995, 2005, and 2015 give the impression that they have been staged to make the woman (and thus the magazine) look as 'good' as possible. However, the change found by Sullivan and O'Connor (1988) was mediated by the increasing depiction of women as decorative objects rather than active participants in images (Sullivan \& O'Connor, 1988). This is interesting as in the current research it is the 1985 


\section{LOOK AT HER: WOMEN IN WOMEN'S MAGAZINES}

images that are the least decorative, yet a study from the same time period (1988) found an increasing trend to depict women as decorative.

Similarly to the results of the current study, in an examination of advertisements from 1979 and 1991, Kang (1997) found no significant change in the overall depiction of gender roles across their timeframe. Lindner (2004) examined advertisements from 1955 to 2002. This research again concluded that the depiction of gender roles was stable across this time period. This result suggests that for Cosmopolitan magazine a conscious decision was made to change the depiction of women on the cover pages in 1985 to reflect less gender stereotypes than 'normal'.

The other notable result from this section of the coding scheme comes not from the inferential analyses but the frequency data. This data show an overwhelming presence of three codes within the sample of cover pages, namely, "feminine touch", "location" and "objectification". The exaggerated presence of these codes within my sample is consistent with the fifth hypothesis of this study that the type of gender stereotyping present in images would be through subtle insinuations rather than overt positioning of dominant male figures. What these three codes have in common is that each of them contributes to the perception of women within images as purely decorative which is a similar finding to the study by Sullivan and O'Connor (1988).

Figure 8 highlights the difference between feminine touch and normal touch. In the image where feminine touch is present the touch is unnatural, it is not touch that would occur in a scenario outside of the staging for this (or another) image. Conversely, the touch exhibited in the second example cover page is a natural position that one may see or enact in an everyday situation. The effect of this makes the coded image appear less natural which adds to the decorative effect of the woman in the image. It is as if she has been arranged for this image. 


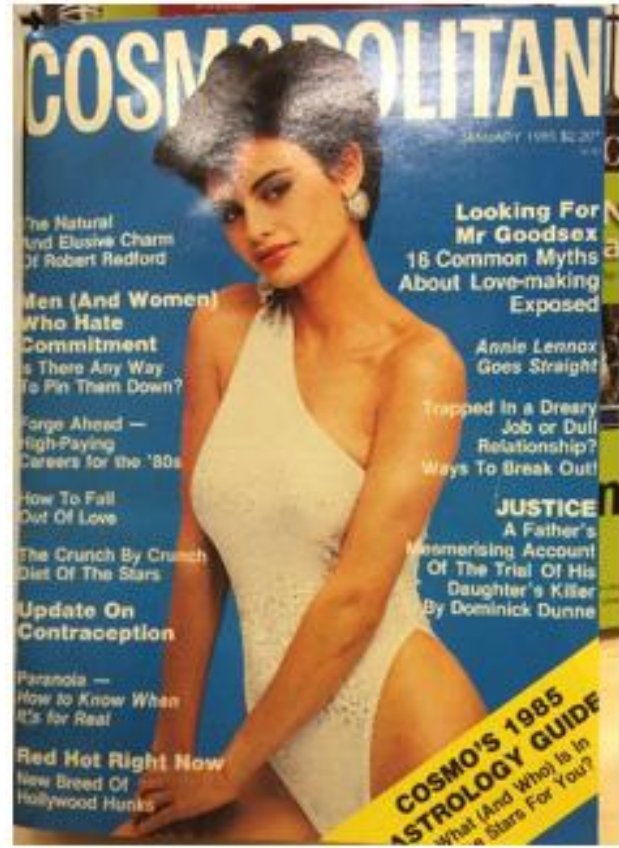

Feminine Touch present

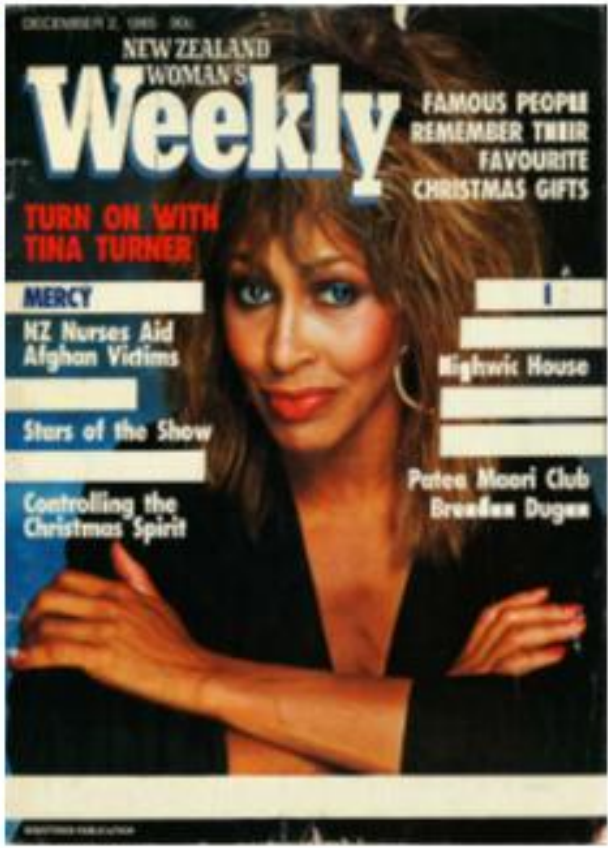

Feminine Touch not present

Figure 8. Example cover pages where feminine touch is present and not present.

Left: From Cosmopolitan Australia January 1985. Copyright 1985 by Bauer Media Pty Limited. Reprinted with permission. Right: From New Zealand Woman's Weekly December $2^{\text {nd }} 1985$. Copyright 1985 by Bauer Media Pty Limited. Reprinted with permission.

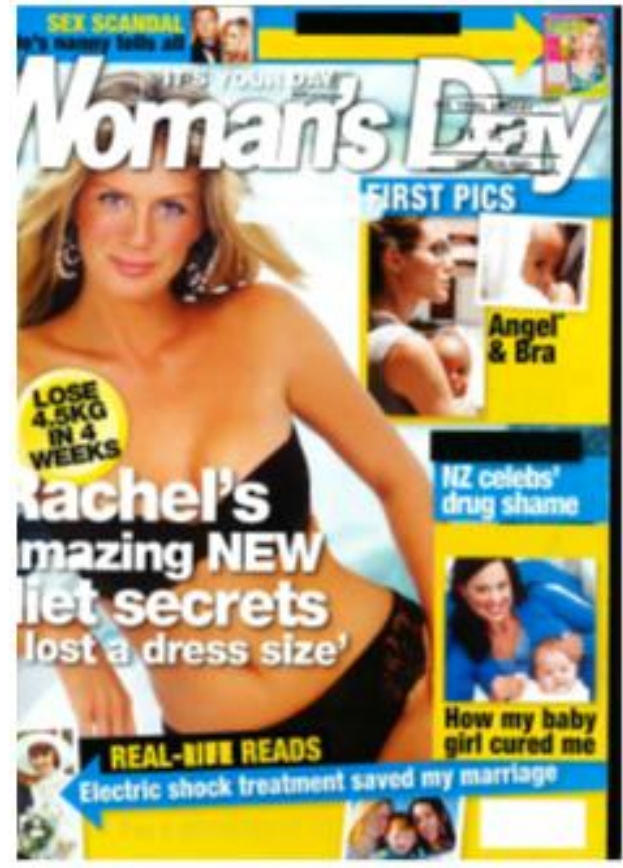

Location coded

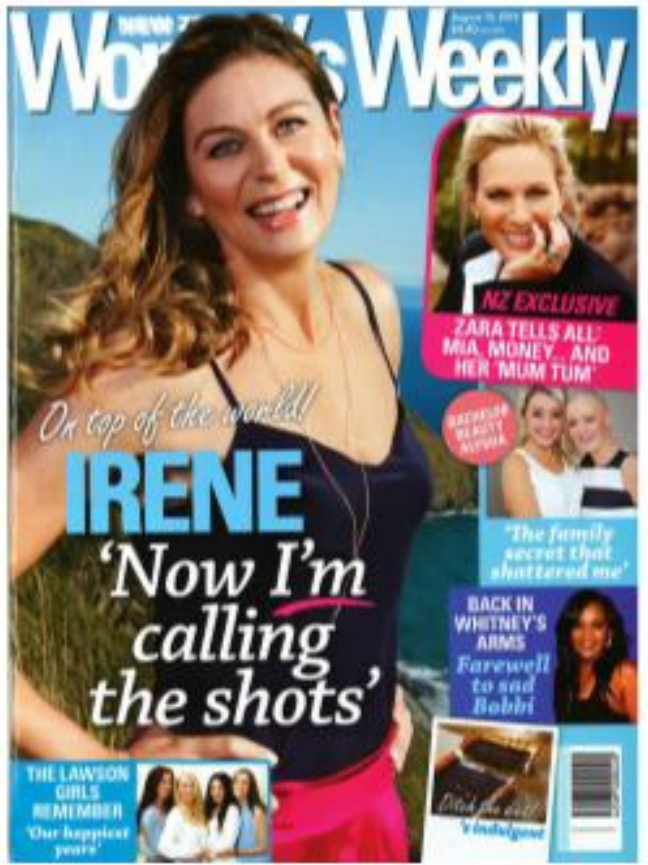

Location not coded

Figure 9. Example cover pages where location was coded for and where it was not coded for.

Left: From New Zealand Woman's Weekly August $1^{\text {st }} 2005$. Copyright 2005 by Bauer Media Pty Limited. Reprinted with permission.

Right: From New Zealand Woman's Weekly August $10^{\text {th }} 2015$. Copyright 2015 by Bauer Media Pty Limited. Reprinted with permission. 
"Location" was coded for when an image featured a woman either in a domestic location (e.g. kitchen or bedroom) or when the setting was unidentifiable (Lindner, 2004). Figure 9 illustrates the difference between when location was coded for and when it was not. As with when 'feminine touch' was coded for, the lack of background in the coded image in Figure 9 depersonalises the example image and again furthers the impression that the woman is there for purely decorative purposes. The non-coded image where the location is identifiable (as an outdoor, hillside, coastal setting) gives some context to the image which also restores some sense of personalisation creating the idea that this person has a life outside of the image. The majority of images where location was coded for were where the setting was unidentifiable, like the example image in Figure 9 (either by being out of focus or because the background was a plain screen). The non-coded image in Figure 9 was the best example from the sample for a lack of this particular feature. Given that in the 'not coded' image the location is still a relatively generic outdoor setting, it highlights how even subtle, personalising changes that restore some sense of agency can have an impact on the interpretation of the image.

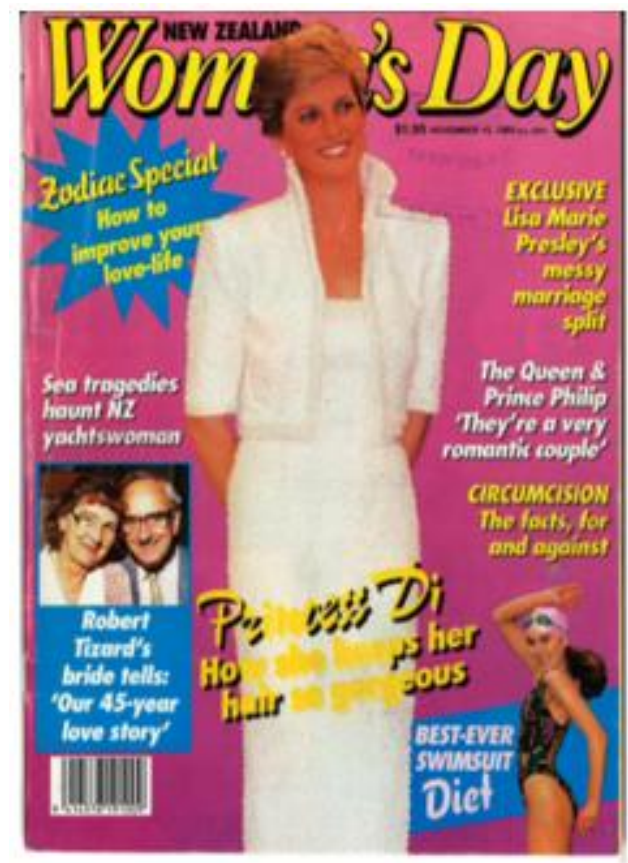

Objectification coded

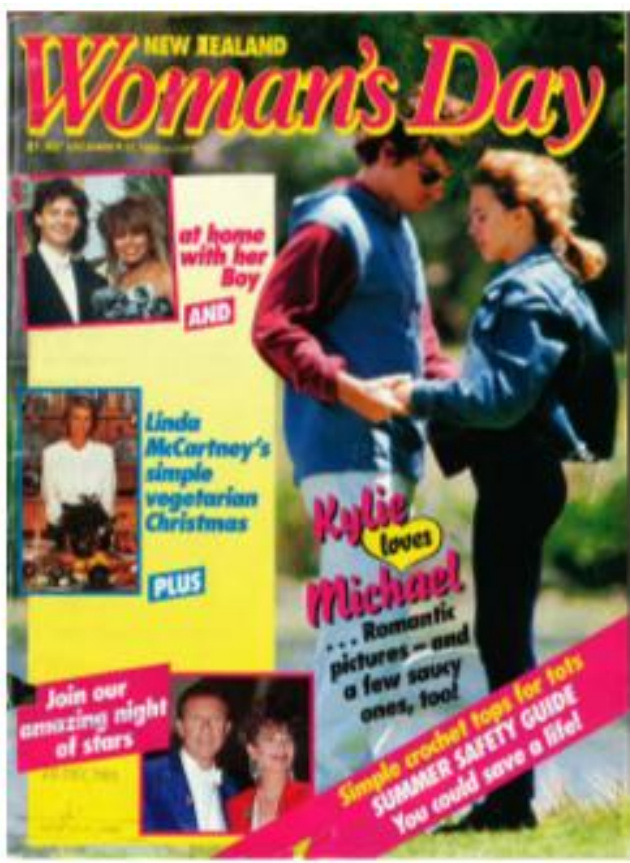

Objectification not coded

Figure 10. Example cover pages where objectification was coded and where it was not coded.

Left: From New Zealand Woman's Day November $15^{\text {th }} 1989$. Copyright 1989 by Bauer Media Pty Limited. Reprinted with permission. Right: From New Zealand Woman's Day December $27^{\text {th }} 1989$. Copyright 1989 by Bauer Media Pty Limited. Reprinted with permission. 
"Objectification" was deemed present in images of women where the purpose of the woman in the image was to be looked at rather than being engaged in any form of focussed activity or additional purpose (e.g. photographs of women playing sports) (Lindner, 2004). Figure 10 illustrates when objectification was coded for and when it was not. Most of the images in the sample that were not coded for objectification were, like the image presented above, where the subject was unaware that they were being photographed at the time the image was being taken. In contrast, the example cover page where objectification was coded for represents images where the subject is being photographed outside of any focussed activity or with any additional purpose aside from being the subject of an image. The difference exhibited in Figure 10, where objectification is present in one image and is not present in the other, is similar to the impression of the other codes identified as agency removing. Where the image is not objectified it appears personal, like the viewer is intruding on a real moment in the life of the subject. Conversely, in the image where objectification is coded for, it is clear that the viewer has the 'right' to look at this image as the purpose of the subject in this image is to be viewed.

The prevalence of these codes is similar to trends found in previous literature. Kang (1997) and Lindner (2004) used coding schedules based on the work of Goffman (1979), which means the features they have coded for are directly comparable to the ones in the current study. Kang (1997) found that the codes "relative size" and "function ranking" were not prevalent within the advertisements sampled from women's magazines. The two codes require the presence of a male figure alongside women in images. "Relative size" refers to the literal domination of space by the man in the image in that it is coded as present if the male figure is larger than the woman (Kang, 1997). "Function ranking" is coded as present if the man in the image is shown in an executive, or controlling, role over the woman (Kang, 1997). Both these codes are overt displays of gender stereotypes that can be depicted in images. The current results also show that these codes are infrequent within the dataset. In addition to this, Kang (1997) also found that the most frequent codes used to convey gender stereotypes were "feminine touch", "licensed withdrawal", and "ritualization of subordination". "Licensed withdrawal" refers to women in images appearing to distance themselves from the scene they are being depicted in (Kang, 1997). "Ritualisation of subordination" refers to either women in images physically lowering/shrinking themselves in images or being depicted using a male counterpart for support in some way (Kang, 1997). The current results show that "ritualisation of subordination" and "licensed withdrawal" were not the most prevalent features used in the depiction of traditional gender roles. However, these two codes are 


\section{LOOK AT HER: WOMEN IN WOMEN'S MAGAZINES}

thematically similar to the current study as they both relate to the removal of agency from women in images.

Lindner (2004), found that the most prevalent codes in their sample of women's

magazines were "feminine touch", "ritualization of subordination", "licensed withdrawal", "location", and "objectification". Again, "relative size" and "function ranking" were infrequently coded in the gendered publication. These codes were also prevalent across the entire timespan of the research (1955-2002) although both "feminine touch" and "licensed withdrawal" were significantly less prevalent in more recent publications (Lindner, 2004). This is interesting as frequency data in the current research shows the opposite pattern, with an increase in the use of feminine touch in 2005 and 2015 (see Table 6). However, the overall finding from Lindner (2004) showing the use of subtle features that promote stereotypes rather than overt ones is similar to the patterns found in the current study.

In sum, parametric analyses showed there were few significant changes in the overall extent of the depiction of gender roles across year groups. The exception to this was within Cosmopolitan magazine, where gender stereotypes were depicted less in cover pages from 1985 when compared to those from 1995, 2005, and 2015. The lack of change across gender role depictions is in line with previous literature and consistent with the third hypothesis, which predicted that there would be a consistent presence of traditional gender roles across the time period. Interesting patterns were found in the frequency data that identify how traditional gender roles are reinforced. The pattern suggests that images of women reflect subtle, insidious, stereotypes about gender. In particular, by excluding depictions of ability, agency, and usefulness in images of women, the damaging notion that women's worth is determined by the way they look rather than by their actions is reinforced.

\section{Implications}

In this section the implications of the findings relating to sexualisation and depiction of gender roles will be discussed. Inferences will be made about what the differences and patterns within the data may mean and how they relate back to the wider context of this thesis, the role of the media in facilitating gender inequality, rape culture, and sexual violence.

\section{Sexualisation}

The results from this research have heralded some interesting trends. Firstly, the results from the current study show that sexualisation on the cover pages of women's magazines is not overt, but is present at a sustained low level across the timespan of this 


\section{LOOK AT HER: WOMEN IN WOMEN'S MAGAZINES}

study. The exception to this is scores from 2005 which show a significant spike in overall sexualisation compared to cover pages from 1975, 1985, and 2015. The results from this research also indicate that monthly 'glossies' (Cosmopolitan, CLEO, and Girlfriend) have higher levels of sexualisation than the weekly publications $N Z W W$ and NZWD.

Finding an overall low level of sexualisation within the current dataset was somewhat contrary to what was expected. The spread within the data indicated that there were high levels of variability within these scores, however, the averages across both year groups and magazines indicate predominantly low scores. Comparisons to the analytical framework outlined by Hatton and Trautner (2011), whose coding scheme was slightly modified for the current research, indicate that whilst the scores were low they can be considered within the "sexualised" range. This pattern has traits that can be linked to the increasing use of microaggressions in society to demean oppressed groups (Sue, 2010). Microaggressions are, as the name suggests, affronts that are covert and brief. They can be delivered both intentionally and unconsciously (Sue, 2010). Microaggressions can cause immense stress for those on the receiving end as they try to process a) whether they have really been insulted, b) whether the offence was purposeful, and c) how best to respond (Kaskan \& Ho, 2016). The low level of sexualisation throughout the sample could be viewed as an example of a microaggression. Rather than an overt display of sexualisation, which women may feel justified to actively criticise, subtlety lessens the risk of alienating the target market while still conveying the message that women are sexual objects.

The most concerning feature about the use of microaggressions in sexualising images is that they can limit the ability for women to respond to them. Covert sexualisation can enable "gaslighting" of women. The term "gaslighting" was coined to describe when one person tries to convince another that their experiences or response to an experience is unwarranted (Abramson, 2014). Due to the fact that microaggressions are fleeting, commonplace, and sometimes unintentional, confronting them can be met with responses of "you're overreacting" and "don’t be so sensitive" (Abramson, 2014, p.1). These dismissive phrases manipulate individuals into thinking that their perception of an experience is wrong and it is a form of silencing (Abramson, 2014). The use of low level sexualisation may therefore be more harmful to gender inequality than overt displays because low level sexualisation not only triggers the same negative responses (such as self-objectification) but women also face more extreme barriers to be able to challenge them. Where barriers to challenging sexual objectification are created it can lead to the sexualisation of women being normalised and this normalisation can contribute to rape culture. If women are framed as 
sexual objects there is an implicit assumption that they are to be used for sex (Calogero et al., 2011). This contributes to rape culture because it is a viewpoint that minimises sexual violence since the idea of a woman's active participation (and therefore active consent) is removed from the framing of sex. In addition to this, the sexualisation of women can have a dehumanising effect (Vance et al., 2015). Loughnan et al. (2013) found that showing sexualised images of women increased victim blaming in hypothetical sexual assault scenarios. These images also had the effect of lessening concern for and the perceived suffering of the female victim. This can further perpetuate rape culture because it contributes to the trivialisation of sexual violence. If an image of a woman in a bikini (Loughnan et al., 2013) can influence the extent to which people care about or blame her in a rape scenario, a normalisation of these images throughout society could well be contributing to these harmful attitudes towards female victims of sexual violence on a wider scale.

Another interesting finding from the inferential results of the current research is that the monthly publications (Cosmopolitan, CLEO, and Girlfriend) have significantly higher levels of sexualisation than the weekly magazines ( $N Z W W$ and $N Z W D)$. This pattern was interesting because despite the fact that all these magazines cater for women, it appears that cover image selection was very different for some than for others. The most notable difference between the higher scoring magazines and the lower scoring magazines (aside from the frequency by which they are published) is that the higher scoring publications target a noticeably younger readership. Cosmopolitan is marketed at 18-35 year olds, CLEO at 1829 year olds, and Girlfriend at 13-19 year olds (Bauer Media New Zealand, 2015; Bauer Media Australia, 2016; Pacific Magazines, 2016). On the other hand, NZWW targets the 40+ age bracket and NZWD has the broadest target demographic, branding itself for women aged 20-49 years old (Bauer Media New Zealand, 2016a, 2016b). The presence of higher sexualisation in publications designed for younger women is concerning as studies have shown that younger women are more susceptible to self-objectification (Szymanski \& Henning, 2007; Tiggemann \& Lynch, 2001). This finding is particularly insidious when other factors are also considered. Firstly, studies have also shown that whilst self-objectification decreases with age, feelings of body dissatisfaction remain stable (Tiggemann \& Lynch, 2001). This means that women have a consistent belief that their appearance is 'not good enough' for their whole lives. Secondly, the frequency data from the current study showed that overwhelmingly the women who were featured on cover pages were in the 18-35 year 
age bracket ${ }^{14}$. This means that in magazines specifically targeting women in the age group most prone to self-objectification, cover images were likely to be sexualised depictions of women from the same age bracket as them. Given that self-objectification is a by-product of comparison (of the self to the presented ideal), age congruence with sexualised cover models could be a reason for increased self-objectification in younger women. This is concerning given the numerous negative outcomes, such as eating disorders, depression, and sexual dysfunction, that have been linked to self-objectification (Aubrey, 2007; Fox, et al., 2015; Fredrickson et al., 1998; Halliwell et al., 2011; Morry \& Staska, 2001).

Further to this, the seemingly specific targeting of a vulnerable population with potentially damaging images can reinforce rape myths and rape culture. Women's magazines are spaces where women are encouraged to step inside and learn how to be feminine and a woman (Burton, 2005; Croteau \& Hoynes, 2014). The images in Cosmopolitan, CLEO and Girlfriend influence the perception of the 'ideal' within the targeted readership of 13-35 year olds. Higher sexualisation scores can therefore imply the construction of the 'ideal' is intertwined with being sexualised. This can facilitate a problematic paradox where women are being encouraged to sexualise themselves but are simultaneously marginalised and punished for that sexualisation. As described in the introduction, there is still a belief in society that 'women ask to be raped' (Burt, 1980; Edwards et al., 2011; Shapcott, 1988). The foundation for this rape myth is that the closer women adhere to traditional notions of femininity, the less at risk they are for sexual violence (Edwards et al., 2011). Appearing sexualised deviates from the 'safety' of femininity and is something that is readily used against women who disclose or report sexual violence. For example, in Scott Kuggeleijn's testimony at his trial for rape (detailed in Chapter 2) he commented that "because of the way she [the victim] was dressed and talked she was the type of girl who loved penis" (Feek, 2016). These mixed messages contribute to the 'impossible space of femininity' (Griffin, Szmigin, Bengry-Howell, Hackley \& Mistral, 2013). This notion, coined in work that examined the drinking patterns in young women (e.g. Griffin et al., 2013; Hutton, Griffin, Lyons, Niland \& McCreanor, 2016; Wright, 2016) refers to the disconnect between behaviour encouraged by post-feminism (participating in activities that only men had been allowed to engage in e.g. drinking alcohol to excess or being sexually assertive) and the expectation to maintain respectability.

\footnotetext{
${ }^{14}$ Sexualisation scores could not be statistically compared across age because the drastically different group sizes prevented a reliable analysis.
} 


\section{LOOK AT HER: WOMEN IN WOMEN'S MAGAZINES}

As discussed earlier, sexualised images in women's magazines can contribute to the normalisation of the idea that women are sexual objects. It can also be inferred that women's magazines encourage women (particularly young women) to sexualise themselves which they are then judged negatively for. Sexualised images of women in women's magazines therefore have the potential to normalise sexual violence against women and also encourage the perception that women who are victims of sexual violence can be held accountable for their own victimisation.

Another interesting difference was also found when mean scores were compared across year groups. Specifically, magazine covers from 2005 were significantly more sexualised than those from 1975, 1985, and 2015. While mean scores show a low level of sexualisation across all year groups, this peak is interesting because it aligns with concerns about the 'pornification' of culture. 'Pornification' is a term used to describe the rising levels of overt depictions of sexualisation in various media forms (Paul, 2005). In media forms directed at women these displays can be packaged as female empowerment, which has been noted in media culture from the mid-1990s onward (Levy, 2005). Sexualisation scores within the current study also begin to rise in 1995, the increase becoming statistically significant for the 2005 year group. It should be noted that this is not a critique of the feminist ideas of sexual liberation - the idea that women should be free to pursue their own sexuality without interference or judgment (hooks, 2015). Rather, it encapsulates the mentality of "why try to beat them when you can join them" (Levy, 2005, p. 93) when it comes to the sexualisation of women. Sexualisation of women is not sexual liberation because it is centred on the idea of being appealing to the male gaze (McCracken, 1993), so these depictions are not expressions of sexual freedom, but instead reflect adherence to views that dehumanise women (Calogero et al., 2011; Smolak \& Murnen, 2011). Encouraging women to adopt this mentality maintains the perception of them as sexual objects which can facilitate gender inequality, rape culture, and sexual violence.

Combining the results from the analyses across publications and across year groups reinforces the idea that the increasing sexualisation of women in the late 1990s to early 2000s is linked to the branding of sexualisation as empowerment. All three magazines with higher sexualisation scores (Cosmopolitan, CLEO and Girlfriend) actively market themselves with themes of empowerment. Marketing for Cosmopolitan states that when read, it "makes women feel like they can take on the world" (Bauer Media Australia, 2016). While still in circulation $C L E O$ was touted as "a true ground breaker for women" (Wilkinson, 2016), and Girlfriend is described as an "innovator" (Pacific Magazines, 2016). These statements may be 


\section{LOOK AT HER: WOMEN IN WOMEN'S MAGAZINES}

true in respect to the content published within the magazine but, as shown by the results of the current study, in regard to their depiction of women on cover pages, they are subscribing to a dangerous trend. Sexualised images influence women to place a greater emphasis on their appearance and the appearance of other women when it comes to determining worth (Szymanski et al., 2011). Normalising sexualisation - which is constructed around the male gaze - not only encourages women to place emphasis on their appearance, but their appearance is inherently tied to satisfying the perceived male expectation (McCracken, 1993). This facilitates gender inequality because it grants men power over the one area that is consistently reinforced as being the sphere in which women can determine their worth (their appearance) (Wolf, 1990). The construction of these power imbalances are implicated time and again in the perpetuation of sexual violence against women (World Health Organisation, 2002; Yodanis, 2004). If women are framed as less than or below men and also depicted in ways that encourages the perception of their gender as sexual objects, this can facilitate a culture where sexual violence is normalised.

Women's magazines play a role in setting the terms for what it means to be a woman. The cover pages of women's magazines depict women in ways that increase their likelihood to be concerned about their appearance, and then women's magazines are filled with advertisements for appearance enhancing products (fashion/beauty) (Burton, 2005; McCracken, 1993; Szymanski et al., 2011). Women's magazines are a key part of industries that flourished with the emergence of women as consumers so it could be posited that the interplay between subliminal messages of the importance of beauty and an abundance of advertisements for beauty products is no coincidence (Wolf, 1990). The backlash against the freedom of women by shackling them to the importance of their appearance can be viewed as a form of social control (Hatton and Trautner, 2013; Wolf, 1990). This is the cycle that has been created for female consumers with little notice paid to the wider consequences. Gender inequality is facilitated; sexualised images of women contribute to perceptions that help sustain rape culture; and within this climate sexual violence against women is endemic (Calogero et al., 2011; World Health Organisation, 2002; Yodanis, 2004). Those benefitting from the depiction of women in this manner are industries that thrive on the influenced insecurities of women. The cosmetics industry generated US $\$ 56.2$ billion in the US alone in 2015 (Sena, 2017). On top of this, the cosmetic surgery industry is currently worth over US $\$ 20$ billion and that value is projected to rise to over US $\$ 27$ billion by 2019 (Bharat Book Bureau, 2015). The fashion industry is worth US $\$ 3$ trillion, this accounts for $2 \%$ of the 
world's GDP ${ }^{15}$ (Fashion United, 2016). These industries have been fairly robust, even showing little downturn in the Global Financial Crisis and subsequent recession of 2008 (Sena, 2017). The emphasis has been placed on making sure that female consumers are consuming and any potential consequences of the negative effects of the way this has been achieved appear to have been overlooked (Lamb, 2015; McCracken, 1993; Wolf, 1990).

Further to this, the socialisation of women to emphasise their appearance for men may be sustaining a divide between genders. Men may not understand the 'obsession' for women to look a certain way and can get frustrated or dismissive about women spending time maintaining their appearance (Wolf, 1990). Men are socialised to expect the 'finished product' of the ideal woman, they are somewhat excluded from the narrative around the steps that one must take to attain it (Wolf, 1990). The frustration at the actual process behind trying to attain this 'ideal' could therefore be frustration towards the idea that the 'ideal' look is normal and easily attainable being challenged. Women on the other hand are aware of the process, products, and time needed to construct this ideal and can feel angry or invalidated by men's dismissive attitude to something that they have spent their lives being taught is of the upmost importance to their success (Wolf, 1990). Images of women in women's magazines may help to reinforce this rhetoric, and the more gender is polarised and these divisions are sustained the further we are as a society from achieving equality.

\section{Gender Roles}

The implications of the prolific use of covert gender stereotypes that remove agency from women in images as well as an interpretation of the differences between depictions in 1985 compared to 1995, 2005, and 2015 within Cosmopolitan magazine are detailed in this section.

The gender roles section of this study indicated a similar pattern to that of the sexualisation results in that a constant presence of features was found across the dataset. The implications of two particularly interesting results will be discussed. Firstly, frequency data indicated that the most commonly used methods of conveying gender stereotypes were in ways that removed agency from women in cover page images. The second notable result was that, for Cosmopolitan magazine, there was a significantly lower level of gender stereotypes within cover images for 1985 when compared to 1995, 2005, and 2015.

\footnotetext{
${ }^{15}$ GDP: gross domestic product, a measure of the monetary value of all goods and services produced in a certain context, in this case the world (Organisation for Economic Development, 2002).
} 


\section{LOOK AT HER: WOMEN IN WOMEN'S MAGAZINES}

The prolific use of gender stereotypes that specifically remove agency from women is a concerning pattern (see Figure 11 for a comparison of images where agency removing codes are present and where they are absent). In the example (Figure 11) where agency has not been removed the woman in the image is shown to have a life and purpose outside of being photographed ${ }^{16}$. In the image where agency is removed (where 'feminine touch', 'location', and 'objectification' have been coded for) a woman is presented who is devoid of purpose outside of being 'decorative'. Images like the 'agency removed' example can encourage general objectification (the treatment of people as things), and when directed towards women this contributes to the harmful rhetoric of gender inequality (Calogero et al., 2011).

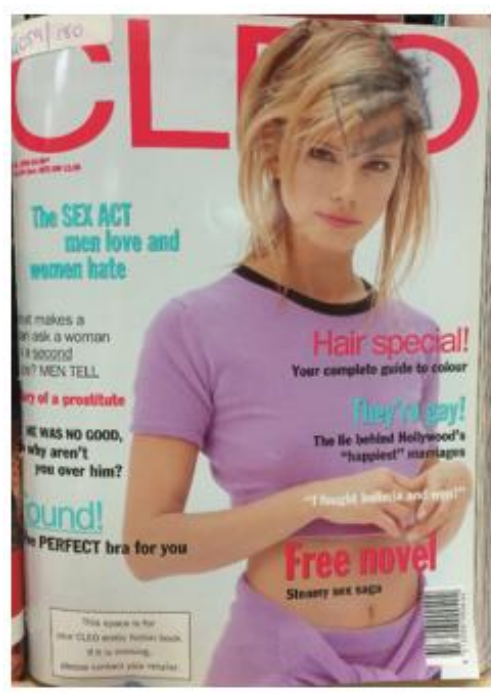

Feminine touch, location, and objectification coded for

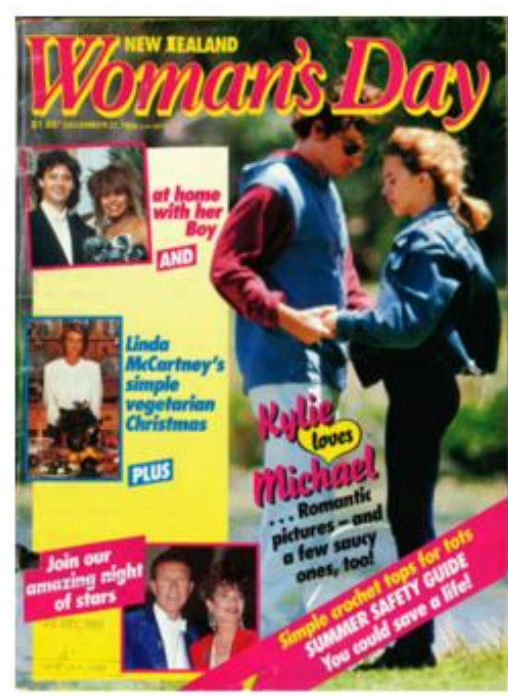

Feminine touch, location, and objectification not coded for

Figure 11. Comparison of images with agency removing codes present and not present.

Left: From CLEO April 1995. Copyright 1995 by Bauer Media Pty Limited. Reprinted with permission. Right: From New Zealand Woman's Day December $27^{\text {th }} 1989$. Copyright 1989 by Bauer Media Pty Limited. Reprinted with permission.

Images hold meaning and they are used to negotiate realities (Sonnevend, 2012). Depictions of women that portray them as display pieces, devoid of purpose and utility, convey the message that for women appearance is revered over ability (Levy, 2005). This concept contributes to gender inequality because it can lead to negative consequences for women. For example, the study mentioned previously, by Tiggemann and Lynch (2001), shows that body dissatisfaction is a constant feature throughout a woman's lifetime. This is

\footnotetext{
${ }^{16}$ In this image 'relative size' was coded for.
} 
one impact of gender stereotypes in images: women spend a lifetime preoccupied with their appearance, perceiving their bodies as inadequate and unappealing because they cannot attain the 'ideal'.

The implications of this are similar to those described in the previous section, namely, the emphasis placed on appearance can lead to a more general view of women as ornamental objects. When a person is objectified (sexually or through a general removal of their agency) it can lead to dehumanised perceptions of them (Vance et al., 2015). When women are dehumanised, violence against them can be trivialised (Loughnan et al. 2013) and the lack of recognition towards the seriousness of violence against women can contribute to rape culture (Buchwald et al., 2005).

What is intriguing is that the results from the current study also show that the depiction of stereotypes can change. Cosmopolitan magazine, which within my dataset has the highest overall level of gender stereotyping ${ }^{17}$, also has the lowest individual year group average of gender role stereotyping with the mean score from $1985^{18}$. It is notable that this occurs in 1985 as during the 1980s feminism and the rights of women were a key talking point in New Zealand society. During this decade, women's issues were actively being discussed in social circles, workplaces, and within government debates (Cook, 2011). The Ministry for Women's Affairs was established in 1984, signalling important recognition for the representation of women's issues in government (Ministry for Women, 2017). Key pieces of legislation were passed that acknowledged the status of women should be equal to men including the amendment to the Crimes Act 1961 that had, up until 1986, prevented women from bringing charges of rape against their husbands (Jordan, 2001). Women were moving into significant positions of power within New Zealand for the first time - Catherine Tizard, for example, became the first woman Mayor of Auckland City in 1984 (and in 1989 she became the first woman Governor-General of New Zealand) (Ministry for Culture and Heritage, 2014). Given this backdrop of significant recognition and advancement for women in New Zealand society at the time, the significantly lower level of gender stereotypes in Cosmopolitan could be a reflection of this. In fact, Wolf (1990) highlights that the economics behind women's magazines were almost toppled by second-wave feminism, with British women's magazines reporting a drop in sales of over 100 million between 1965 and 1981 . Women's magazines were out of touch and a change was needed in order for them and their related industries to survive (Wolf, 1990). Thus came the strategic rebranding to what was

\footnotetext{
${ }^{17}$ This is not a statistically significant result.

${ }^{18}$ This is not a statistically significant result.
} 


\section{LOOK AT HER: WOMEN IN WOMEN'S MAGAZINES}

currently of interest to women (liberation) but as magazines were also a place to entice female consumers to the associated industries, liberation had to be something that could be bought (Wolf, 1990). Women were momentarily afforded some reprieve from traditional gender roles but something that Wolf (1990) refers to as The Beauty Myth was being constructed - the idea that success could and would be measured by a woman's appearance. What these results show is that images of women can change as a potential reflection of their standing in society. Unfortunately, the level of gender role stereotypes has increased steadily over the time periods following 1985. This reinforces the idea that the change was superficial rather than a meaningful change in attitudes towards women.

Interpreting the pattern of lower gender roles in Cosmopolitan during 1985 as a reflection of prevailing attitudes at the time makes the resurgence of traditional gender roles in 1995, 2005 and 2015 all the more concerning. One could infer that the change in 1985 was at a surface level, appeasement for consumers that reflected popular opinion at the time. This could explain why, when the status of women became less of a talking/selling point, old habits were resurrected because, on a deeper, more fundamental level, attitudes towards women were unchanged. Given the knowledge about the links between the status of women and the perpetration of sexual violence against them (Yodanis, 2004), this is a particularly insidious implication.

This section discussed the concerns around the abundant use of agency removing, gender stereotyping features in the cover page images and how these contribute to a wider climate of gender inequality. Further, the implications of fluctuations in gender role depictions within Cosmopolitan magazine were discussed as reflections of the status of women's equality in society at the time of publication. Given this trend (lower in 1985 and rising across 1995, 2005, and 2015), inferences were made about what this could mean in respect to gender inequality.

\section{Applications}

This section details the practical applications of the findings from this research. This includes its contribution to academic literature, the importance of identifying and framing ideas of gender inequality in a medium that is accessible and relatable, and how women's magazines could potentially create a platform that would have a positive influence on the lives of their target audience.

The findings of this thesis contribute to the base of feminist academic literature that aims to highlight the ways in which gender inequality is reinforced by various mechanisms 


\section{LOOK AT HER: WOMEN IN WOMEN'S MAGAZINES}

within society. Women's magazines are a media type exclusively designed for that gender, and what they convey can have an influence on women in negotiating their femininity (Burton, 2005; Croteau \& Hoynes, 2014). This research places tropes of inequality in a medium that many women are familiar with and encounter on a regular basis (waiting rooms or the conveniently located racks at the checkouts of most supermarkets). Locating ideas of gender inequality in recognisable mediums brings challenging issues to a more relatable context. This is all the more important given the idea that the sexual objectification of women is the product of a systemic backlash against the gains of second-wave feminism (Hatton and Trautner, 2013; Wolf, 1990). Findings suggest the achievements and success of women are routinely explained by their appearance or undermined by a polarising focus on what they look like rather than what they have achieved (Hatton and Trautner, 2013). In this climate women, particularly young women, who were born after the second-wave feminist movement in the midst of this backlash, need the tools to recognise and resist this system of oppression. Academic research has shown the profound effects that sexualising images can have on challenging gender inequality (Calogero, 2013). Images could therefore not only be contributing to inequality but they could also be affecting the movement to fight against that inequality. In this climate, where inequality has become more subtle and those who are affected by it are discouraged from engaging with informative narratives, research that equips women with the abilities to recognise tools of oppression becomes critically important.

Those who contribute to the content of women's magazines are not naïve to the influence they can have on the lives of their target audience. The purpose of the cover page is to sell the publication under the banner that the magazine will inform readers of women's issues and guide them in what it means to be a woman and further, what it means to be the 'ideal' woman (Burton, 2005; Croteau \& Hoynes, 2014). What magazines are doing, however, is manipulating their readership to overemphasise the importance of their appearance. This can lead to negative feelings about the self which not only keeps women reliant on women's magazines to advise them on how to be a better woman but also garners more interest for the products on sale within the pages of the publication (McCracken, 1993; Szymanski et al., 2011). This is certainly an effective business model - and looking at the trillions of dollars that the beauty, fashion, and cosmetic industries are worth it is hard to make an argument otherwise. But what if women's magazines changed their ways and focussed on actually empowering readers? What if instead of presenting women as 'decorative' they are afforded agency and personality when they are photographed? What if, instead of sexualising women, they are depicted in personalised, meaningful ways? It begs 


\section{LOOK AT HER: WOMEN IN WOMEN'S MAGAZINES}

the question: what would a feminist image look like? From my sample I do not have an image that scored ' 0 ' on both scales so I cannot display an example and that in itself speaks volumes about the pervasiveness of these patterns in women's magazines. Considering the potential harms connected to the way women's magazines depict women both historically and currently, changing the tone surely warrants consideration. However, challenging such an ingrained norm has proven not to be so easy. In the recent body-positive documentary, Embrace (Brumfitt \& Vincent, 2016), Mia Freedman, former editor of Cosmopolitan Australia, describes trying to change the culture by doing a lingerie fashion shoot with plus size models:

"My fashion editor was horrified and then she kept coming to me and saying 'I can't get any clothes'... clothing designers don't want their brand associated with anyone larger than a size $8 \ldots$ Eventually she did it [got some clothes] and the photographer didn't want his name on the shoot, the make-up artist didn't want her name on the shoot. It was a real battle!"

Mia Freedman, in Brumfitt \& Vincent, 2016.

What is highlighted particularly well in this quote is the interconnectedness of the fashion/beauty industry with women's magazines (McCracken, 1993; Wolf, 1990). This lessens my optimism for the ability for women's magazines to orchestrate change themselves. Women's magazines do not operate as independent entities; it is not enough for a feminist editor to try and evoke change because the interconnected industries will create barriers at every opportunity. For real change to occur it would require a transformation in mind-set across multiple industries and given the proven lucrativeness of the current business model, this seems unlikely.

The findings from this thesis contribute to the body of literature on the actual and potential harms of sexualised and stereotypical portrayals of gender in women's magazines. It is hoped that it may inspire change in the industry but that may be unlikely. The idea that women are sexual objects, devoid of agency, is disturbingly commonplace in media for women. This barrier to change may also be affecting the ability to achieve gender equality and abolish rape culture. 


\section{Chapter 6: Conclusion}

The previous chapter detailed the findings from this research, placing the results into context and drawing inferences from this data. It was found that women's magazines can contribute to the normalisation of the sexual objectification of women, they depict harmful gender stereotypes that encourage the perception of women as 'decorative' and it was argued that these things can contribute to gender inequality, rape culture, and sexual violence. In this chapter, a brief summary of the study and main findings is given before an overall conclusion and ideas for future research directions are discussed.

\section{Summary}

The aim of this thesis was to investigate how women are depicted on the cover pages of New Zealand women's magazines across a 40 year time period. It was predicted that depictions of women would be sexualised and that the level of sexualisation would increase over the time span. The portrayal of traditional gender roles in images of women were predicted to be present and consistent across the 40 year time period and that when present they would remove agency from women in images, thus depicting them as 'decorative'.

The results from this research showed a low level of sexualisation within the sample overall and that the level of sexualisation peaked in 2005. Magazines with a younger readership (Cosmopolitan, CLEO, and Girlfriend) had significantly higher levels of sexualisation than those for older audiences. Scores from the gender roles section of the coding scheme showed a consistent presence of traditional gender roles in images across the 40 year time span. The most frequently used codes were those that reflected a removal of agency from women in images. The only deviation from this was for cover pages from Cosmopolitan in 1985, where significantly lower levels of gender stereotypes were depicted in the images.

Sexualisation and the depiction of traditional gender roles on the covers of women's magazines do not exist in vacuum. Magazine cover pages are designed within a wider context where it has been established that it is acceptable to depict women in decorative, sexualised ways. That context is in turn reinforced by the existence of those cover pages and they reflect the 'norms' that women are decorative, sexual objects. Challenging these 'norms' that do nothing but contribute to inequality is imperative and ideally women's magazines would take more responsibility for what they convey to their audiences. Perpetuating the idea that women are sexual objects or that their worth is measured against their adherence to the 


\section{LOOK AT HER: WOMEN IN WOMEN'S MAGAZINES}

'ideal' appearance can lead to women becoming complicit in their own oppression (Calogero, 2013). Viewing women as lesser than men is an idea central to sustaining rape culture. This idea can enable the minimisation of sexual violence as the primary victims are dehumanised (Vance, et al., 2015).

\section{Future Research}

The cover page is a key feature to any magazine but it is not the only image within a publication that suggests and reinforces ideas about the 'ideal'. Future research should consider widening the analysis to include all images of the woman depicted on the cover page. This could include a more detailed look at how the cover figure is depicted within the magazine. Having spent a fair amount of time surrounded by women's magazines, I would tentatively suggest that there could be some interesting findings here. Feature articles seem to include a medley of photos of the cover image subject, some of these have been, at a glance, more sexualised than the selected cover photo. Also, most of these images tend to place emphasis on appearance rather than depicting (often) successful women in the arenas where they have earned their success (e.g. actresses in paparazzi shots rather than on set or performing). This could be quite a topical piece given the relatively recent objections from celebrity women about the emphasis placed on their appearance rather than their talent in media interviews (Wong, 2016). Actress Scarlett Johansson, for example, has refused to answer questions on how she got into shape for her role in The Avengers franchise and has highlighted that her male co-stars are asked about their acting preparation whereas the focus for her is on her physical appearance in the films (Wong, 2016). Johansson appears on the cover of one of the magazines in my sample (see Figure 12) - this cover page scored '14' for sexualisation (one of the highest scoring in the sample) and ' 4 ' for gender roles. Scarlett Johansson is the highest grossing actress of all time and the only woman to feature in the list of top ten grossing actors (Guerrasio, 2016), yet her appearance is still the topic interviewers and magazine covers choose to highlight. This reinforces the idea from Hatton and Trautner (2013) that women's successes are undermined or ignored in favour of emphasising their appearance. There is the potential that images within the magazine could further illustrate this idea. 


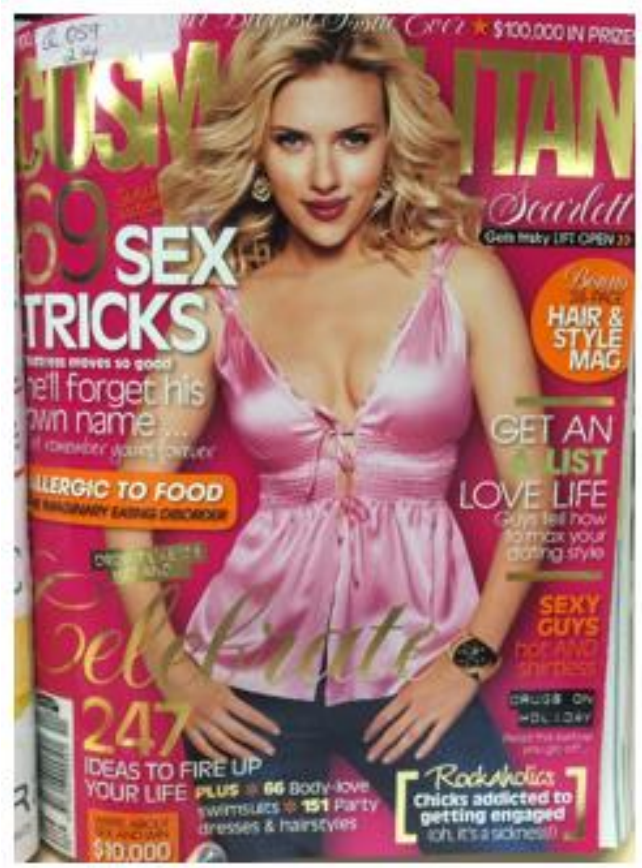

Cosmopolitan cover page November 2005, featuring Scarlett Johansson

Figure 12. Cover image from Cosmopolitan magazine featuring Scarlett Johansson.

Cover page: From Cosmopolitan Australia November 2005. Copyright 2005 by Bauer Media Pty Limited. Reprinted with permission.

On a similar thread, textual analysis from the cover image's corresponding article could provide even greater detail as to the depiction of the woman within the image. Past studies have shown that written articles about women can also endorse traditional gender roles and sexual objectification. In a comparative analysis of Sports Illustrated and Sports Illustrated for Women, it was found that in feature articles about women athletes $20 \%$ of stories were unrelated to sport. In comparison, only $8 \%$ of stories about male athletes discussed non-sporting content (Fink \& Kensicki, 2002). A future study could examine the written content of feature articles in women's magazines to discover the extent to which the focus of the articles is sexualised, or whether it endorses traditional gender stereotypes.

Further to this, the rise of social media presents a new platform by which people are engaging with ideas. An interesting avenue for research looking at depictions of women would be a social media analysis of how women are discussed, portrayed or targeted as consumers in this new media form. Could it be that this new media form has new ideas and trends in how women are represented? Or, has the pattern of sustaining old ideas within new mediums continued? These are avenues worth exploring to ensure that a holistic knowledge of how women are portrayed is maintained. 
LOOK AT HER: WOMEN IN WOMEN'S MAGAZINES

\section{Conclusion}

As recognised by international agencies such as the United Nations, sexual violence against women is a global epidemic and needs to be assessed and addressed from all angles if it is to be remedied. The presence of sexualisation and traditional gender roles within the current data present further hurdles to challenging sexual violence. The pervasive presence of these features over time, through significant changes to the social status of women, signifies that there is an undercurrent of harmful attitudes that has yet to be addressed. Further to this, the results from this thesis suggest that gender inequality is being normalised in a media form designed to convey influential information about what it means to be a woman primarily to women. The use of images that are tied to promoting inequality within the group that is being oppressed is inherently problematic as it reinforces ideas that, if internalised, can influence women to be complicit in maintaining their secondary status. Women's magazines need to take more responsibility for the influence they may have on their readers but given the extent to which these harmful patterns have already been normalised, it appears that for now, society will continue to simply 'look at her'. 


\section{References}

Abrahams, N., Devries, K., Watts, C., Pallitto, C., Petzold, M., Shamu, S., \& García-Moreno, C. (2014). Worldwide prevalence of non-partner sexual violence: a systematic review. The Lancet, 383(9929), 1648-1654.

Abramson, K. (2014). Turning up the Lights on Gaslighting. Philosophical Perspectives, 28(1), 1-30.

Aubrey, J. S. (2007). The impact of sexually objectifying media exposure on negative body emotions and sexual self-perceptions: Investigating the mediating role of body selfconsciousness. Mass Communication \& Society, 10(1), 1-23.

Aubrey, J. S. (2010). Looking good versus feeling good: An investigation of media frames of health advice and their effects on women's body-related self-perceptions. Sex Roles, 63(1-2), 50-63.

Baker, C. N. (2005). Images of women's sexuality in advertisements: A content analysis of Black-and White-oriented women's and men's magazines. Sex roles, 52(1), 13-27.

Barrington, R. (1986). Rape law reform. Women's Studies International Forum, 9(1), 57-61.

Bates, L. (2015, May 22). How School Dress Codes Shame Girls and Perpetuate Rape Culture. Time. Retrieved March 20, 2017, from http://time.com/3892965/everydaysexism-school-dress-codes-rape-culture/

Bauer Media Australia. (2016). Brands - Cosmopolitan Australia. Retrieved October 2, 2015, from http://www.bauer-media.com.au/brands/cosmopolitan/

Bauer Media New Zealand. (2015). Brands - CLEO. Retrieved October 2, 2015, from http://www.bauermedia.co.nz/brands/cleo

Bauer Media New Zealand. (2016a). Brands - The New Zealand Woman's Weekly. Retrieved October 2, 2015, from http://www.bauermedia.co.nz/brands/new-zealand-womansweekly?page $=1 \&$ node $=5510 \&$ exclude $=\&$ module $=5516 \&$ page Size $=0 \&$ items $=0 \&$ pagi $\underline{\text { ng }=\text { tru }}$

Bauer Media New Zealand. (2016b). Brands - Woman's Day. Retrieved October 2, 2015, from http://www.bauermedia.co.nz/brands/womansday?page $=0 \&$ node $=5510 \&$ module $=5516 \&$ page Size $=0 \&$ items $=0 \&$ paging $=$ true 
Bell, P. (2001). Content analysis of visual images. In T. van Leeuwen \& C. Jewitt (Eds.), Handbook of visual analysis (pp.10 - 34). Wolverhampton UK: SAGE.

Bharat Book Bureau. (2015). Global Cosmetic Surgery and Service Market Report 20152019. Retrieved April 7, 2017 from http://www.prnewswire.com/newsreleases/global-cosmetic-surgery-and-service-market-report-2015-2019295910691.html

Bohner, G., \& Schwarz, N. (1996). The threat of rape: Its psychological impact on nonvictimized women. In D.M. Buss \& N.M. Malamuth (Eds.), Sex, power, conflict: Evolutionary and feminist perspectives (pp. 162 - 175). New York, NY: Oxford University Press.

Bourke, J. (2007). Rape: Sex, Violence, History. Emeryville, CA: Shoemaker \& Hoard.

Brownmiller, S. (1975). Against our will: Men, women and rape. New York, NY: Simon \& Schuster.

Brumfitt, T. (Producer/Director), \& Vincent, A. (Producer). (2016). Embrace [Motion Picture]. Australia: Body Image Movement.

Buchwald, E., Fletcher, P. R., \& Roth, M. (Eds.). (2005). Transforming a rape culture. Minneapolis, MN: Milkweed Editions.

Burt, M. R. (1980). Cultural myths and supports for rape. Journal of personality and social psychology, 38(2), $217-230$.

Burton, G. (2005). Media and society: Critical perspectives. UK: McGraw-Hill Education.

Calogero, R., Tantleff-Dunn, S., \& Thompson, J. (2011). Objectification Theory: An Introduction. In R. Calogero, S. Tantleff-Dunn \& J. Thompson (Eds.), SelfObjectification in Women: Causes, Consequences, and Counteractions (pp. 3 -21). Washington, DC, US: American Psychological Association.

Calogero, R. M. (2013). Objects Don't Object: Evidence That Self-Objectification Disrupts Women's Social Activism. Psychological Science, 24(3), 312 - 318.

Carmody, M., \& Carrington, K. (2000). Preventing sexual violence? Australian \& New Zealand Journal of Criminology, 33(3), 341-361. 
Carrabine, E. (2012). Just images aesthetics, ethics and visual criminology. British Journal of Criminology, 52(3), 463-489.

Cohn, A. M., Zinzow, H. M., Resnick, H. S., \& Kilpatrick, D. G. (2013). Correlates of reasons for not reporting rape to police: Results from a national telephone household probability sample of women with forcible or drug-or-alcohol facilitated/incapacitated rape. Journal of interpersonal violence, 28(3), 455-473.

Collins, P. H. (2015). Intersectionality's definitional dilemmas. Annual Review of Sociology, $41,1-20$.

Colson-Smith, R. N. (2005). Look Younger, Lose 10 Pounds, and Influence Your Audience: A Content Analysis of Popular Men's and Women's Magazine Cover Blurbs and the Messages They Project to Their Readers. (Master of Arts Thesis, East Tennessee State University, Tennessee, United States of America). Retrieved from http://dc.etsu.edu/etd/1001/ (1001)

Cook, M. (2011). Page 6. The women's liberation movement. Retrieved March 8, 2017, from http://www.TeAra.govt.nz/en/womens-movement/page-6

Crimes Act. (1961). Retrieved from New Zealand Legislation, http://www.legislation.govt.nz/act/public/1961/0043/latest/DLM329051.html

Croteau, D., \& Hoynes, W. (2013). Media/society: Industries, images, and audiences: Sage Publications.

D’Cruze, S. (Ed.) (2011). Sexual violence in history: A contemporary heritage. London: Routledge.

Dietz, T. L. (1998). An examination of violence and gender role portrayals in video games: Implications for gender socialization and aggressive behavior. Sex Roles, 38(5-6), 425-442.

Dooney, L. (2017, March 8). Year 9s at St Patrick's Silverstream suspended over sexual harassment of staff. Stuff. Retrieved March 17, 2017, from http://www.stuff.co.nz/national/education/90179350/Year-9s-at-St-Patricks$\underline{\text { Silverstream-suspended-over-sexual-harassment-of-staff }}$ 
Dougan, P. (2013, November 5). Roast Busters: Radio show hosts 'victim blaming. New Zealand Herald. Retrieved August 30, 2016, from http://www.nzherald.co.nz/nz/news/article.cfm?c id=1\&objectid=11151911

Eagly, A. H. (2009). The his and hers of prosocial behavior: an examination of the social psychology of gender. American Psychologist, 64(8), 644 - 658.

Edwards, A., \& Heenan, M. (1994). Rape trials in Victoria: Gender, socio-cultural factors and justice. Australian \& New Zealand Journal of Criminology, 27(3), 213-236.

Edwards, K. M., Turchik, J. A., Dardis, C. M., Reynolds, N., \& Gidycz, C. A. (2011). Rape myths: History, individual and institutional-level presence, and implications for change. Sex Roles, 65(11-12), 761-773.

Ericson, R. V. (1991). Mass Media, Crime, Law, and Justice-An Institutional Approach. British Journal of Criminology, 31, 219 - 249.

Evidence Act. (2006). Retrieved from New Zealand Legislation, http://www.legislation.govt.nz/act/public/2006/0069/latest/DLM393463.html

Fanslow, J. L., \& Robinson, E. (2004). Violence against women in New Zealand: prevalence and health consequences. The New Zealand Medical Journal, 117(1206).

Fashion United. (2016). Global Fashion Industry Statistics. Retrieved April 7, 2017 from https://fashionunited.com/global-fashion-industry-statistics

Feek, B. (2016, August 1). Rape accused acted like any man: lawyer. New Zealand Herald. Retrieved January 21, 2017, from http://m.nzherald.co.nz/nz/news/article.cfm?c_id=1\&objectid=11685150

Field, A. (2009). Discovering statistics using SPSS. London, UK: SAGE publications.

Fink, J. S., \& Kensicki, L. J. (2002). An imperceptible difference: Visual and textual constructions of femininity in Sports Illustrated and Sports Illustrated for Women. Mass Communication \& Society, 5(3), 317-339.

Finley, L. L. (2016). Domestic abuse and sexual assault in popular culture. Santa Barbara, CA: ABC-CLIO. 
Fox, J., Ralston, R. A., Cooper, C. K., \& Jones, K. A. (2015). Sexualized avatars lead to women's self-objectification and acceptance of rape myths. Psychology of women quarterly, 39(3), $349-362$.

Fraser, C. (2015). From Ladies First to Asking for It: Benevolent Sexism in the Maintenance of Rape Culture. California Law Review, 103, 141 - 204.

Frederick, D. A., Forbes, G. B., Grigorian, K. E., \& Jarcho, J. M. (2007). The UCLA Body Project I: Gender and ethnic differences in self-objectification and body satisfaction among 2,206 undergraduates. Sex Roles, 57(5-6), 317-327.

Fredrickson, B. L., \& Roberts, T. A. (1997). Objectification theory: Toward understanding women's lived experiences and mental health risks. Psychology of women quarterly, 21(2), $173-206$.

Fredrickson, B. L., Roberts, T.-A., Noll, S. M., Quinn, D. M., \& Twenge, J. M. (1998). That swimsuit becomes you: sex differences in self-objectification, restrained eating, and math performance. Journal of personality and social psychology, 75(1), 269 - 284.

Frith, K., Shaw, P., \& Cheng, H. (2005). The Construction of Beauty: A Cross-Cultural Analysis of Women's Magazine Advertising. Journal of communication, 55(1), 5670.

Gavey, N. (2005). Just sex?: The cultural scaffolding of rape. Sussex, U.K.: Routledge.

Goffman, E. (1979). Gender advertisements. New York, NY: Harper \& Row.

Greer, C. (2007). News media, victims and crime. In P. Davies, P. Francis, C. Greer (Eds.), Victims, crime and society (pp. 20 - 49). London, U.K.: SAGE Publications.

Griffin, M. (2010). Media images of war. Media, War \& Conflict, 3(1), 7-41.

Griffin, C., Szmigin, I., Bengry-Howell, A., Hackley, C., \& Mistral, W. (2013). Inhabiting the contradictions: Hypersexual femininity and the culture of intoxication among young women in the UK. Feminism \& Psychology, 23(2), 184-206.

Guerrasio, J. (2016, June 30). Scarlett Johansson is now the highest-grossing female movie star ever. Business Insider Australia. Retrieved April 7, 2017, from https://www.businessinsider.com.au/scarlett-johansson-highest-grossing-female-starever-2016-6 


\section{LOOK AT HER: WOMEN IN WOMEN'S MAGAZINES}

Haboush, A., Warren, C. S., \& Benuto, L. (2012). Beauty, ethnicity, and age: Does internalization of mainstream media ideals influence attitudes towards older adults? Sex Roles, 66(9-10), 668-676.

Halliwell, E., Malson, H., \& Tischner, I. (2011). Are contemporary media images which seem to display women as sexually empowered actually harmful to women? Psychology of women quarterly, 35(1), 38-45.

Hatton, E., \& Trautner, M. N. (2011). Equal opportunity objectification? The sexualization of men and women on the cover of Rolling Stone. Sexuality \& culture, 15(3), 256-278.

Hatton, E., \& Trautner, M. N. (2013). Images of powerful women in the age of "choice feminism'. Journal of Gender Studies, 22(1), 65-78.

hooks, b. (2000). Feminist theory: From margin to center. London, U.K.: Pluto Press.

Hutton, F., Griffin, C., Lyons, A., Niland, P., \& McCreanor, T. (2016). “Tragic girls” and "crack whores": Alcohol, femininity and Facebook. Feminism \& Psychology, 26(1), 73-93.

Hsieh, H. F., \& Shannon, S. E. (2005). Three approaches to qualitative content analysis. Qualitative health research, 15(9), 1277-1288.

Jackson, S., \& Scott, S. (2002). Gender: A sociological reader. London, U.K.: Routledge.

Jewkes, R. (2002). Intimate partner violence: causes and prevention. The Lancet, 359(9315), 1423-1429.

Jewkes, R., Flood, M., \& Lang, J. (2015). From work with men and boys to changes of social norms and reduction of inequities in gender relations: a conceptual shift in prevention of violence against women and girls. The Lancet, 385(9977), 1580-1589.

Jewkes, Y. (2004). Media and crime. London, U.K.: SAGE Publications.

Johnson, R. R. (2014). Rape and gender conflict in a patriarchal state. Crime \& Delinquency, 60(7), 1110-1128.

Jordan, J. (2001). True" lies" and False" truth"": Women, Rape and the Police. (Doctoral Thesis, Victoria University of Wellington, Wellington, New Zealand).

Jordan, J. (2012). Silencing rape, silencing women, in J. Brown \& S. Walklate (Eds.), Handbook on Sexual Violence (pp. 253-286). London: Routledge. 
Kachel, S., Steffens, M. C., \& Niedlich, C. (2016). Traditional masculinity and femininity: Validation of a new scale assessing gender roles. Frontiers in Psychology, 7, 1 - 19.

Kang, M. E. (1997). The portrayal of women's images in magazine advertisements: Goffman's gender analysis revisited. Sex roles, 37(11), 979-996.

Kang, S. (2016, March 21). Outrage after Paula Bennett told a 'bit of sexual violence never hurt anyone'. New Zealand Herald. Retrieved September 1, 2016, from http://www.nzherald.co.nz/nz/news/article.cfm?c id=1\&objectid=11608971

Kaskan, E. R., \& Ho, I. K. (2016). Microaggressions and female athletes. Sex Roles, 74(7-8), 275-287.

Kaur, R. (2015). milk and honey. Kansas City, Missouri: Andrews McMeel Publishing.

Kelly, L. (2013). Surviving sexual violence. Oxford, U.K.: Polity Press.

Kinser, A. E. (2004). Negotiating spaces for/through third-wave feminism. NWSA journal, $16(3), 124-153$.

Kolbe, R. H., \& Burnett, M. S. (1991). Content-analysis research: An examination of applications with directives for improving research reliability and objectivity. Journal of consumer research, 18(2), 243-250.

Krassas, N. R., Blauwkamp, J. M., \& Wesselink, P. (2003). "Master your Johnson”: Sexual rhetoric in Maxim and Stuff magazines. Sexuality and Culture, 7(3), 98-119.

Krippendorff, K. (1989). Content Analysis. In E. Barnouw, G. Gerbner, W. Schramm, T.L., Worth, \& L. Gross (Eds.), International encyclopedia of communication (pp. 403). New York, NY: Oxford University Press.

Lambiase, J., \& Reichert, T. (2006). Sex and the marketing of contemporary consumer magazines: How men's magazines sexualized their covers to compete with Maxim. In T. Reichert, J. Lambiase (Eds.), Sex in consumer culture: The erotic content of media, marketing (pp. 67 - 86). Mahwah, NJ: Erlbaum.

Levy, A. (2006). Female chauvinist pigs: Women and the rise of raunch culture. New York, NY: Simon and Schuster.

Lindner, K. (2004). Images of women in general interest and fashion magazine advertisements from 1955 to 2002. Sex Roles, 51(7-8), 409-421. 
Lonsway, K. A., \& Fitzgerald, L. F. (1994). Rape myths in review. Psychology of women quarterly, 18(2), 133-164.

Loughnan, S., Pina, A., Vasquez, E. A., \& Puvia, E. (2013). Sexual objectification increases rape victim blame and decreases perceived suffering. Psychology of women quarterly, $37(4), 455-461$.

Lynskey, D. (2013, November 13). Blurred Lines: the most controversial song of the decade. The Guardian. Retrieved March 20, 2017, from https://www.theguardian.com/music/2013/nov/13/blurred-lines-most-controversial$\underline{\text { song-decade }}$

Macnamara, J. R. (2005). Media content analysis: Its uses, benefits and best practice methodology. Asia-Pacific Public Relations Journal, 6(1), 1-34.

Mahdawi, A. (2016, October 15). This is what rape culture looks like - in the words of Donald Trump. The Guardian. Retrieved January 21, 2017, from https://www.theguardian.com/us-news/2016/oct/15/donald-trump-words-what-rapeculture-looks-like

Malone, A. K., Florence; Hinton, Marc; Edens, John; Robinson, Georgina. (2015, August 8). Chiefs allegedly exposed themselves to stripper. Stuff. Retrieved January 21, 2017, from http://www.stuff.co.nz/sport/rugby/82852409/chiefs-allegedly-exposedthemselves-to-stripper

McCracken, E. (1993). Decoding Women's Magazines: From Mademoiselle to Ms. Basingstoke, U.K.: Springer.

McDonald, E. (2014). From "Real Rape" to Real Justice? Reflections on the Efficacy of More Than 35 Years of Feminism, Activism and Law Reform. Victoria University of Wellington Law Review, 45, 487-508.

Milburn, M. A., Mather, R., \& Conrad, S. D. (2000). The effects of viewing R-rated movie scenes that objectify women on perceptions of date rape. Sex Roles, 43(9-10), 645664.

Ministry for Culture and Heritage. (2014). Dame Catherine Tizard. Retrieved March 20, 2017 from https://nzhistory.govt.nz/media/photo/dame-catherine-tizard 
LOOK AT HER: WOMEN IN WOMEN'S MAGAZINES

Ministry for Women. (2017). Ministry for Women - About Us, Ministry for Women.

Retrieved January 24, 2017 from http://women.govt.nz/about/new-zealandwomen/history/ministry

Ministry of Justice. (2007). The New Zealand Crime and Safety Survey 2006: Key Findings. Retrieved from:

http://ndhadeliver.natlib.govt.nz/delivery/DeliveryManagerServlet?dps_pid=IE76350 $\underline{3}$

Ministry of Justice. (2010). The New Zealand Crime and Safety Survey: 2009 Main Findings Report. Retrieved from:

http://ndhadeliver.natlib.govt.nz/delivery/DeliveryManagerServlet?dps_pid=IE33385 $\underline{29}$

Ministry of Justice. (2015). 2014 New Zealand Crime and Safety Survey: Main Findings. Retrieved from:

https://www.justice.govt.nz/assets/Documents/Publications/NZCASS-201602-MainFindings-Report-Updated.pdf

Ministry of Social Development. (2017). Sexual Violence Service Development Update. New Zealand. Retrieved from http://www.msd.govt.nz/webadmin/html/enews/svsdu$\underline{\text { march-24-2017.html }}$

Morry, M. M., \& Staska, S. L. (2001). Magazine exposure: Internalization, selfobjectification, eating attitudes, and body satisfaction in male and female university students. Canadian Journal of Behavioural Science/Revue canadienne des sciences $d u$ comportement, 33(4), $269-279$.

Mossman, E., Jordan, J., MacGibbon, L., Kingi, V., \& Moore, L. (2009). Responding to Sexual Violence: a review of literature on good practice. Wellington, New Zealand: Ministry of Women's Affairs.

Muehlenkamp, J. J., Swanson, J. D., \& Brausch, A. M. (2005). Self-objectification, risk taking, and self-harm in college women. Psychology of women quarterly, 29(1), 2432. 
New Zealand Tourism Guide. (2016). New Zealand Seasons. New Zealand Weather and Climate. Retrieved September 1, 2015 from http://www.tourism.net.nz/newzealand/about-new-zealand/weather-and-climate.html

Organisation for Economic Cooperation and Development, (2002). Gross Domestic Product. Retrieved April 7, 2017 from http://stats.oecd.org/glossary/detail.asp?ID=1163

Organisation for Economic Cooperation and Development, (2016). Violence Against Women. Retrieved September 2, 2016 from https://data.oecd.org/inequality/violenceagainst-women.htm

Pacific Magazines. (2016). Girlfriend (NZ). Retrieved, October 2, 2015, from http://www.pacificmags.com.au/Pages/Magazines/Magazine.aspx?mid=fe16ab4 1-1358-4925-b418-96d5d593388a

Paul, P. (2005). Pornified: How pornography is transforming our lives, our relationships, and our families. New York, NY: Macmillan.

Pawlowski, I. P. (2007). Sex in Women's Magazine Advertising: An analysis of the degree of sexuality in women's magazine advertising across age demographics and women's responses. (Masters thesis, University of Canterbury, Christchurch, New Zealand).

Reichert, T., Lambiase, J., Morgan, S., Carstarphen, M., \& Zavoina, S. (1999). Cheesecake and beefcake: No matter how you slice it, sexual explicitness in advertising continues to increase. Journalism \& Mass Communication Quarterly, 76(1), 7-20.

Reiner, R. (2007). Media made criminality: the representation of crime in the mass media. In R. Reiner, M. Maguire, R. Morgan, (Eds.) The Oxford Handbook of Criminology (pp. 302-340). Oxford, U.K.: Oxford University Press.

Richins, M. L. (1991). Social comparison and the idealized images of advertising. Journal of consumer research, 18(1), 71-83.

Ryan, K. M. (2011). The relationship between rape myths and sexual scripts: The social construction of rape. Sex Roles, 65(11-12), 774-782.

Saada Saar, M. (2014, September 12). Grand Theft Auto V and the Culture of Violence Against Women. The Huffington Post. Retrieved January 22, 2017, from http://www.huffingtonpost.com/malika-saada-saar/grand-theft-auto-v-and-the-cultureof-violence-against-women_b_6288528.html 
Sable, M. R., Danis, F., Mauzy, D. L., \& Gallagher, S. K. (2006). Barriers to reporting sexual assault for women and men: Perspectives of college students. Journal of American College Health, 55(3), 157-162.

Sachdeva, S. (2016, September 8). NZ Rugby investigation into Chiefs stripper scandal 'bogus', politicians say. Stuff. Retrieved, January 21, 2017, from http://www.stuff.co.nz/national/politics/84036784/NZ-Rugby-investigation-intoChiefs-stripper-scandal-bogus-politicians-say

Schwalbe, C., \& Dougherty, S. (2015). Visual coverage of the 2006 Lebanon War: Framing conflict in three US news magazines. Media, War \& Conflict, 8(1), 141-162.

Schwarz, N., \& Kurz, E. (1989). What's in a picture? The impact of face-ism on trait attribution. European Journal of Social Psychology, 19(4), 311-316.

Sena, M. (2017). Beauty Industry Analysis 2017 - Cost \& Trends. Retrieved April 7, 2017 from https://www.franchisehelp.com/industry-reports/beauty-industry-report/

Shapcott, D. (1988). The Face of the Rapist: Why men rape - the myths exposed. Auckland, N.Z.: Penguin.

Shim, J. W., Kwon, M., \& Cheng, H.-I. (2016). Analysis of representation of sexuality on women's and men's pornographic websites. Social Behavior and Personality: an international journal, 44(3), 53-62.

Smolak, L., \& Murnen, S. K. (2011). The Sexualisation of Girls and Women as a Primary Antecedent of Self-Objectification. In R.M. Calogero, S. Tantleff-Dunn, K.J. Thompson (Eds.) Self-objectification in women: Causes, consequences, and counteractions (pp. 53-75). Washington, DC, U.S.: American Psychological Association.

Soley, L., \& Kurzbard, G. (1986). Sex in advertising: A comparison of 1964 and 1984 magazine advertisements. Journal of Advertising, 15(3), 46-64.

Sonnevend, J. (2012). Iconic Rituals: towards a Social Theory of encountering Images. In J. Alexander, D. Bartmanski, \& B. Giesen (Eds.), Iconic power: materiality and meaning in social life (pp.219-232). New York, NY: Palgrave Macmillan. 
Statistics New Zealand. (2013). 2013 Census QuickStats about national highlights. New Zealand. Retrieved from http://m.stats.govt.nz/Census/2013-census/profile-andsummary-reports/quickstats-about-national-highlights/age-and-sex.aspx.

Stewart, M. (2017, March 10). Rape culture action moved after Wellington College boys threaten protesters. Stuff. Retrieved March 19, 2017, from http://www.stuff.co.nz/national/education/90272223/rape-culture-action-moved$\underline{\text { after-wellington-college-boys-threaten-protesters }}$

Sue, D. W. (2010). Microaggressions in everyday life: Race, gender, and sexual orientation. Hoboken, NJ: John Wiley \& Sons.

Sullivan, G. L., \& O'Connor, P. J. (1988). Women's role portrayals in magazine advertising: 1958-1983. Sex roles, 18(3), 181-188.

Szymanski, D. M., \& Henning, S. L. (2007). The role of self-objectification in women's depression: A test of objectification theory. Sex Roles, 56(1-2), 45-53.

Szymanski, D. M., Moffitt, L. B., \& Carr, E. R. (2011). Sexual objectification of women: advances to theory and research. The Counseling Psychologist, 39(1), 6-38.

Thorpe, I. (2017, March 13). No 'rape culture' at Wellington College. Stuff. Retrieved March 20, 2017 from http://www.stuff.co.nz/stuff-nation/assignments/rape-in-nz-join-thedebate/17717053/No-rape-culture-at-Wellington-College

Thursdays in Black. (2016). Thursdays in Black, Aotearoa - About. Retrieved October 2, 2016 from http://www.thursdaysinblack.org.nz/about

Tiggemann, M., \& Lynch, J. E. (2001). Body image across the life span in adult women: the role of self-objectification. Developmental psychology, 37(2), 243 - 253.

Tolman, D. L. (1994). Doing desire: Adolescent girls' struggles for/with sexuality. Gender \& Society, 8(3), 324-342.

Trotter, C. (2013, November 8). How have we raised sons like these? Stuff. Retrieved September 20, 2016 from http://www.stuff.co.nz/dominionpost/comment/columnists/chris-trotter/9375328/How-have-we-raised-sons-like-these

Umiker-Sebeok, J. (1996). Power and construction of gendered spaces. International Review of Sociology, 6(3), 389-403. 
United Nations Women. (2016a). Facts and figures: Ending violence against women.

Retrieved September 20 2016, from http://www.unwomen.org/en/what-we-do/endingviolence-against-women/facts-and-figures\#notes

United Nations Women. (2016b). HeForShe UN Women Solidarity Movement for Gender Equality [Press release]. Retrieved September 20 2016, from http://www.heforshe.org//media/heforshe/files/our\%20mission/heforshe_overview_brief.pdf

Vance, K., Sutter, M., Perrin, P. B., \& Heesacker, M. (2015). The media's sexual objectification of women, rape myth acceptance, and interpersonal violence. Journal of Aggression, Maltreatment \& Trauma, 24(5), 569-587.

Viera, A. J., \& Garrett, J. M. (2005). Understanding interobserver agreement: the kappa statistic. Family Medicine, 37(5), 360-363.

Watt, D. P. (2012). The Urgency of Visual Media Literacy in Our Post-9/11 World: Reading Images of Muslim Women in the Print News Media. Journal of Media Literacy Education, 4(1), 32-43.

Wilkinson, L. (2016, January 20). I Never Wanted To Be Editor Of Cleo... But I'm So Proud I Was. The Huffington Post Australia. Retrieved March 20, 2017, from http://www.huffingtonpost.com.au/lisawilkinson/i-never-wanted-to-be-editor-ofcleobut-am-so-proud-iwas_b_9005358.html?utm_content=bufferf4636\&utm_medium=social\&utm_source= twitter.com\&utm_campaign=buffer

Williams, K. (2017, March 16). Wellington College students stood down over Facebook 'rape' comments. Stuff. Retrieved March 20, 2017 from http://www.stuff.co.nz/national/education/90481818/wellington-collegestudents-suspended-over-facebook-rape-comments

Wolf, N. (1990). The Beauty Myth: How Images of Beauty Are Used Against Women. London, U.K.: Vintage.

Wong, K. (2016). 8 Times Female Celebrities Brilliantly Shut Down Sexist Interview Questions. Retrieved March 30, 2017, from https://mic.com/articles/141019/8-timesfemale-celebrities-brilliantly-shut-down-sexist-interview-questions\#.vjVzOAncW 
LOOK AT HER: WOMEN IN WOMEN'S MAGAZINES

World Economic Forum. (2016). Global Gender Gap Report 2016. Retrieved from http://reports.weforum.org/global-gender-gap-report-2016/rankings/

World Health Organisation. (2002). World Report on Violence and Health. Geneva, Switzerland: World Health Organization.

World Health Organisation. (2013). Global and regional estimates of violence against women: prevalence and health effects of intimate partner violence and non-partner sexual violence. Geneva, Switzerland: World Health Organization.

Wright, S. (2016). 'Serious public mischief': young women, alcohol and the New Zealand press. Continuum, 30(6), 636-645.

Yodanis, C. L. (2004). Gender inequality, violence against women, and fear a cross-national test of the feminist theory of violence against women. Journal of Interpersonal Violence, 19(6), 655-675. 


\section{Appendices}

\section{Appendix A Code Book}

\section{Code Book}

\section{General Definitions}

Main Image - defined as the main photograph of the cover page, usually bigger than all other images and related to the main headline/caption (also typically biggest on page aside from name of magazine)

Feature Article - article within the magazine issue that relates to the cover image. Where there is no directly related article the feature article is the one referenced in the main caption on the cover page.

Feature Article Main Image - the largest image accompanying the feature article that is different to the photo used on the cover page. Where multiple images fit this criterion the first image that fits the previous criteria is to be used.

\section{Part 1 - Demographic Information}

This part of the coding scheme is to capture demographic information, in which there may be longitudinal trends of interest to this research. Six decisions need to be made for this part of the coding scheme.

1. Single person vs. Group shot

Single person: main cover image features one individual person

Group shot: main cover image feature more than one individual

\section{Gender}

There are two tiers to this decision the first applies where there is a single person in the image, the second to where there is a group

Single: person to be coded as male or female

Group: coded as mixed gender (male(s) and female(s) present), all female, or all male

3. Age

Age is grouped into 5 bands and is an approximation based on appearance - can be informed by prior knowledge of the person within the image (e.g. if a celebrity's age is known, that can determine the age band rather than your approximation).

$>18$ : younger than 18

18-25 years old

26-35 years old

36-50 years old

$50+$ years old

4. Ethnicity

The ethnicity coding is based around ethnicities that we see in NZ as the magazines are mostly NZ based publications. Individuals should be coded to this even if they are known to be from overseas (e.g. American, English etc.). Judgment based on what NZ ethnicity they best represent.

New Zealand European: of European descent, Caucasian 
Maori

Pacific Islander

African American

Asian

Indian

Other: name if possible (e.g. Hispanic)

5. Local vs. International

Judgement based on prior knowledge (e.g. the Queen is international, Judy Bailey is from New Zealand etc.), can use external sources if unsure (e.g. Googling celebrity name)

Local: someone who is from/lives in New Zealand and/or Australia (included because some magazines are published in Australia and so 'local' to them must be included here)

International: someone from outside of NZ/Australia N/A: where this information is unknown/unavailable

6. Public Figure vs. Cover Model

Something ambiguous that could come up when making this decision is if someone is a famous model (e.g. Rachel Hunter, Kate Moss, or Gigi Hadid). People who fit this category should be coded as 'public figure' as they are a known entity.

Public figure: celebrity, known personality, famous

Cover model: non-celebrity, unknown personality

\section{Part 2 - Captions}

This part of the coding scheme looks at the written captions on the cover page, with the decisions that need to be made in this section being about grouping the caption into themes. This decision can be tricky as some captions may appear to fit into two categories, need to decide where the focus of the text is, can use other prompts to help decide (e.g. if caption is related to an image). Coded numerically for ease. Don't code competition/prize captions.

1. Beauty/Body Image - promotes physical self-image, includes 'how-to guides on beauty/make up', potential for crossover with "health" (e.g. Look good and get healthy), should be scrutinised as to whether the focus is actually health or about looking a certain way.

2. Career - oriented toward professional issues like advancing in the workplace, interview tips etc., NOT things like workplace fashion. Also includes (with Girlfriend magazine in particular (younger audience)) captions relating to study.

3. Celebrity Infatuation - text presenting celebrity in sexual or lustful manner, scandal headlines, focus on image

4. Celebrity news - text pertaining to current celebrity issues, information that isn't typically scandalised or sexualised (e.g. Queen's royal tour, Blake Lively talks about motherhood)

5. Family - promoting family relationship issues, raising children, issues with siblings/parents 
6. Fashion - captions relating to fashion trends and topics (e.g. get their look!)

7. Health - captions relating to personal health, includes mental health issues and healthy eating/diets, find your true self/self-help type headlines

8. Home - focussing on domestic issues (e.g. cooking, cleaning, decorating, flatmates)

9. Love/relationship - text relating to relationship issues and/or finding love and/or maintaining relationships

10. Marriage - text relating to marital topics - can be closely related to "Family", is a more specific category must be related to weddings/marital issues/in laws/divorce

11. Money - focussing on financial theme, can appear to cross over (e.g. Get the latest fashion without breaking the bank) judgement needs to be made about focus of statement

12. News - text relating to current events (outside of the world of celebrity news, incl. 'real life' stories)

13. Sex - text focussed on sexual gratification (outside of love/relationships and/or marriage, includes "Hot guy" sections/objectification of males)

14. Leisure - text relating to activities/hobbies including sports, reading, craft, travel etc.

\section{Part 3 - Image: Sexualisation}

The sexualisation part of the image coding has 10 categories, each category is scored on a sliding scale and the individual scores are then added and an overall sexualisation score is formed for the image as a whole. Each code has a different scale range and higher scores reflect a higher level of sexualisation within that code. The sexualisation coding scheme is to be done on the woman within the cover image (if it is a group shot with multiple women then a judgment must be made as to which is the focus of the image, e.g. largest within shot, in foreground of shot, caption relates to one woman over the other). If there is no woman on the cover page then this part of the coding scheme does not need to be completed.

Clothing/Nudity - refers to the level of dress

$0=$ unrevealing clothing

$1=$ modest clothing (e.g. low necklines, exposed arms/shoulder)

$2=$ moderately revealing (e.g. exposed midriffs, shorts - for one half of the body (e.g. shorts with t-shirt or exposed midriff with longer skirt)

$3=$ highly revealing (e.g. skin tight clothing, plunging necklines)

4= clothed in apparel most would not consider clothing (e.g. lingerie, swimwear)

$5=$ naked or minimal clothing (e.g. socks/shoes).

Touch - refers to self-touch, touching others, and being touched

$0=$ no touch present

$1=$ casual touching (e.g. clasping own hands, resting arm on shoulder of another person)

$2=$ provocative touching (touching self or others in a way that suggest potential sexual contact e.g. hand on thigh of another) 
$3=$ sexually explicit touching (touching/covering of an intimate area (breasts, chest, buttocks, genitals).

Pose - refers to the positioning of the subject

$0=$ pose in no way related to sexual activity (e.g. standing or engaged in innocuous activity)

$1=$ suggested/invited sexual activity (e.g. lifting arms overhead in absence of activity that would require this, any kind of sitting/leaning)

$2=$ overt positioning for sexual activity (e.g. lying down, leg(s) spread, inviting/drawing attention to crotch).

Mouth - refers to the subjects mouth, how it is positioned/held

$0=$ no suggestion of sexual activity (e.g. closed lips, broad smiles, active use of mouth (e.g. talking, singing))

$1=$ somewhat sexualised (e.g. slightly parted lips)

$2=$ explicitly sexual mouth positioning (e.g. passive wide open mouths, tongue showing, object in mouth)

Facial Expression - refers to the relationship suggested between model and viewer based on the model's gaze

$0=$ demand/affiliation - model holds direct line of eye contact with viewer, smiling or not smiling

$1=$ offer/ideal - model offers self as idealised version, gaze is directed away from viewer

$2=$ demand/submission - the 'come hither' expression, model looks down at viewer, not smiling

$3=$ demand/seduction - active expression of sexual desire, model looks up at viewer, tilted head, smiling or pouting

Breasts - in regards to whether this part of the body is the focal point of the shot

$0=$ not visible/not focal point

$1=$ image is focal point but fully covered

$2=$ body part is focal point and is exposed/suggestive of exposure (e.g. unbuttoning of clothes)

Genitals - in regards to whether this part of the body is the focal point of the shot

$0=$ not visible/not focal point

$1=$ image is focal point but fully covered

$2=$ body part is focal point and is exposed/suggestive of exposure (e.g. unbuttoning of clothes)

Buttocks - in regards to whether this part of the body is the focal point of the shot

$0=$ not visible/not focal point

$1=$ image is focal point but fully covered 
$2=$ body part is focal point and is exposed/suggestive of exposure (e.g. unbuttoning of clothes)

Head/Body Shot - whether the image includes just a close up of the face or a full shot of the body

$0=$ head shot (including images that are of the head and shoulders)

$1=$ body shot

Text - relates to the caption directly linked to the main image

$0=$ absences of any sort of sexual innuendo

$1=$ caption contains some sort of sexual innuendo

$2=$ explicit reference to sex or sexuality

\section{Part 4 - Image: Gender Roles}

If there is no woman on the cover page then this part of the coding scheme does not need to be completed. Focus should be on the same woman used for the sexualisation part of the coding scheme.

Relative Size - when depicted alongside men, women are typically smaller/take up less space within the image

$\mathrm{Y}=$ feature is present, male within image take up more space than female

$\mathrm{N}=$ feature isn't present, women takes more space or equal space within the image compared to the man

$\mathrm{NA}=$ cannot code for the feature (e.g. when both genders aren't present in the image)

Function Ranking - when both gender are depicted the man is shown as the one in control, or a more powerful/executive role

$\mathrm{Y}=$ feature is present, male within image is in control/more powerful role

$\mathrm{N}=$ feature isn't present, women is in control or there is no difference between genders

$\mathrm{NA}=$ cannot code for the feature (e.g. when both genders aren't present)

Feminine Touch - where women are positioned to have unnatural touch with their own bodies or objects, unusual defined as detached from how that object/body part would be touched/used in a real-life scenario

$\mathrm{Y}=$ feature is present, women unusually touching self/object, touch is often gentle/caressing rather than controlling/manipulating an object

$\mathrm{N}=$ feature isn't present, woman is touching self/object in normal manner

$\mathrm{N} / \mathrm{A}=$ no touch of self/object in image

Ritualization of Subordination - women demonstrates some sort of lowering/shrinking of the body (e.g. lying down, canting postures, sitting). Can also be coded if woman is being restricted by or leaning on/supported by male counterpart in image

$\mathrm{Y}=$ feature is present

$\mathrm{N}=$ feature isn't present 
Licensed Withdrawal - woman must appear to psychologically remove herself from the situation/context of the image (e.g. vacant expression, covering face/mouth, withdrawal of gaze, engaging in activity out of larger context of image).

$\mathrm{Y}=$ feature is present

$\mathrm{N}=$ feature isn't present

Body Display - where a woman's body is displayed by way of revealing/minimal/no clothing $\mathrm{Y}=$ feature is present

$\mathrm{N}=$ feature isn't present

Movement - where a woman is inhibited in her movement (e.g. wrapped in a blanket)

$\mathrm{Y}=$ feature is present

$\mathrm{N}=$ feature isn't present

Location - woman is shown in a domestic location (e.g. kitchen, bedroom, or bathroom) or she is in a decontextualized setting that can't be identified for any specific/purposeful activities

$\mathrm{Y}=$ feature is present

$\mathrm{N}=$ feature isn't present

Objectification - purpose of the woman in the image is to be looked at; woman is not engaged in any focussed activity

$\mathrm{Y}=$ feature is present

$\mathrm{N}=$ feature isn't present 
LOOK AT HER: WOMEN IN WOMEN'S MAGAZINES

\section{Appendix B Coding Checklist}

\begin{tabular}{|c|c|c|}
\hline & Coding Checklist & \\
\hline & Category & Ranking \\
\hline & Single person vs. Group Shot (Single $=1$, Group $=2$ ) & \\
\hline & Gender (Single: Female=1, Male=2, Group: Mixed gender $=3$, All male =4, All female $=5$ ) & \\
\hline & Age $(>18=0$ 18-25=1, 26-35=2, 36-50=3, 50+=4) & \\
\hline & Ethnicity (NZ Euro=1, Maori=2, Pl=3, AfrAm=4, Asian=5, Ind=6, Other=7) & \\
\hline & Local vs. International (Local=1, Int=2, N/A =3) & \\
\hline & Public Figure vs. Cover Model (PubFig=1, CovMod=2) & \\
\hline & Posed vs. Papparazzi (Pose=1, Pap=2) & \\
\hline \multirow{13}{*}{ 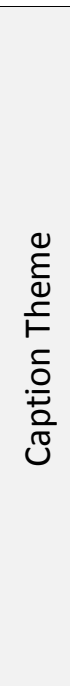 } & Caption Theme (1-14 code, see code book) & \\
\hline & Main Caption & \\
\hline & Image Caption & \\
\hline & Sub Caption & \\
\hline & Sub Caption & \\
\hline & Sub Caption & \\
\hline & Sub Caption & \\
\hline & Sub Caption & \\
\hline & Sub Caption & \\
\hline & Sub Caption & \\
\hline & Sub Caption & \\
\hline & Sub Caption & \\
\hline & Sub Caption & \\
\hline \multirow{10}{*}{ 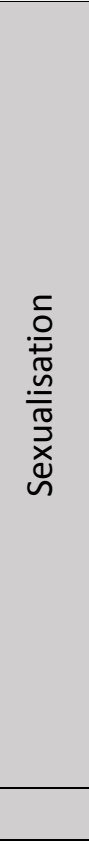 } & Clothing/Nudity (0-5 points) & \\
\hline & Touch (0-3 points) & \\
\hline & Pose (0-2 points) & \\
\hline & Mouth (0-2 points) & \\
\hline & Facial Expression (0-3 points) & \\
\hline & Breasts (0-2 points) & \\
\hline & Chest (0-2 points) & \\
\hline & Genitals (0-2 points) & \\
\hline & Buttocks (0-2 points) & \\
\hline & Head vs. Body Shot (0-1 point) & \\
\hline
\end{tabular}


LOOK AT HER: WOMEN IN WOMEN'S MAGAZINES

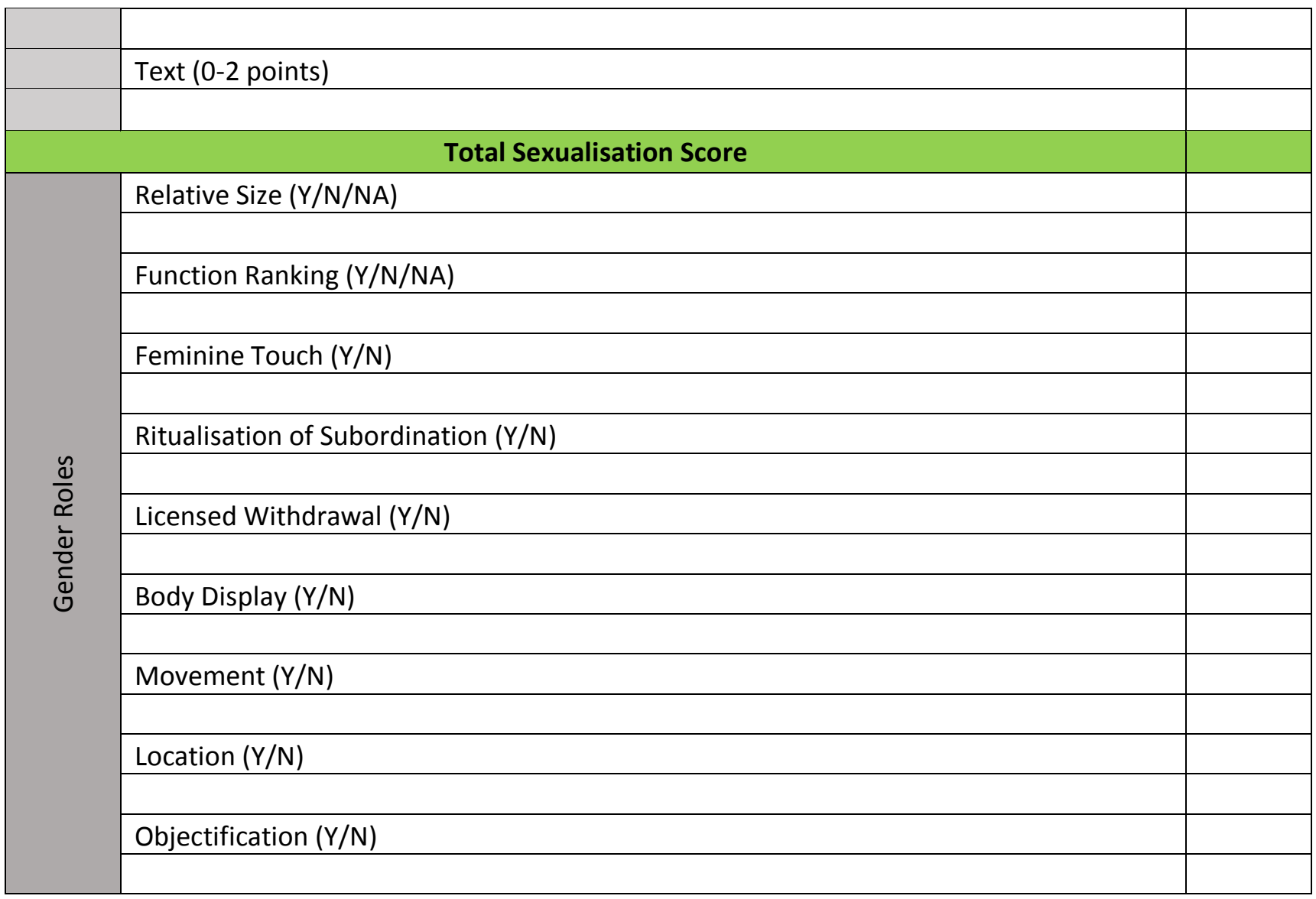

